University of Tennessee Health Science Center

UTHSC Digital Commons

$12-2014$

\title{
Downregulation of Prdm16 Is Critical for HOXB4-mediated Benign HSC Expansion In Vivo
}

Hui Yu

University of Tennessee Health Science Center

Follow this and additional works at: https://dc.uthsc.edu/dissertations

Part of the Medical Genetics Commons, and the Medical Molecular Biology Commons

\section{Recommended Citation}

Yu, Hui , "Downregulation of Prdm16 Is Critical for HOXB4-mediated Benign HSC Expansion In Vivo" (2014). Theses and Dissertations (ETD). Paper 307. http://dx.doi.org/10.21007/etd.cghs.2014.0367.

This Dissertation is brought to you for free and open access by the College of Graduate Health Sciences at UTHSC Digital Commons. It has been accepted for inclusion in Theses and Dissertations (ETD) by an authorized administrator of UTHSC Digital Commons. For more information, please contact jwelch30@uthsc.edu. 


\title{
Downregulation of Prdm16 Is Critical for HOXB4-mediated Benign HSC Expansion In Vivo
}

\author{
Abstract \\ Overexpression of HOXB4 in hematopoietic stem cells (HSCs) leads to increased self-renewal without \\ causing hematopoietic malignancies in transplanted mice. The molecular basis of HOXB4-mediated \\ benign HSC expansion in vivo is not well understood. To gain further insight into the molecular events \\ underlying HOXB4-mediated HSC expansion, we analyzed gene expression changes at multiple time \\ points in Lin-Sca1+c-kit+ (LSK) cells from mice transplanted with bone marrow (BM) cells transduced \\ with a MSCV-HOXB4-ires-YFP vector. A distinct HOXB4 transcriptional program was reproducibly induced \\ and stabilized by 12 weeks after transplant. Dynamic expression changes were observed in genes critical \\ for HSC self- renewal as well as genes involved in myeloid and B cell differentiation. Prdm16, a \\ transcription factor associated with human acute myeloid leukemia (AML), was markedly repressed by \\ HOXB4 but upregulated by HOXA9 and HOXA10, suggesting that Prdm16 downregulation was involved in \\ preventing leukemia in HOXB4 transplanted mice. Functional evidence to support this mechanism was \\ obtained by enforcing co-expression of sPrdm16 and HOXB4, which led to myeloid expansion, and \\ leukemia. Before the onset of leukemia decreased HSC frequency was observed in HOXB4and sPrdm16 \\ co-expressing cells, suggesting sustained expression of Prdm16 abolished HOXB4-mediated HSC \\ expansion. Altogether, these studies define the transcriptional pathways involved in HOXB4 HSC \\ expansion in vivo and identify repression of Prdm16 transcription as a mechanism by which HOXB4 \\ mediates a benign HSC expansion.

\section{Document Type} \\ Dissertation

\section{Degree Name} \\ Doctor of Philosophy (PhD)

\section{Program} \\ Biochemistry \\ Research Advisor \\ Brian P. Sorrentino, MD

\section{Keywords} \\ Hematopoietic Stem Cell, HOXB4, Leukemogenesis, Prdm16, Self-renewal

\section{Subject Categories} \\ Medical Genetics | Medical Molecular Biology | Medical Sciences | Medicine and Health Sciences
}


DOWNREGULATION OF PRDM16 IS CRITICAL FOR HOXB4-MEDIATED BENIGN HSC EXPANSION IN VIVO

\author{
A Dissertation \\ Presented for \\ The Graduate Studies Council \\ The University of Tennessee \\ Health Science Center
}

\author{
In Partial Fulfillment \\ Of the Requirements for the Degree \\ Doctor of Philosophy \\ From The University of Tennessee
}

By

Hui $\mathrm{Yu}$

December 2014 
Copyright (C) 2014 by Hui Yu All rights reserved 


\section{DEDICATION}

This dissertation is dedicated to my parents, Guoquan $\mathrm{Yu}$ and $\mathrm{Yan} \mathrm{Xu}$, my husband, Satish Kumar Nandakumar and my precious daughter Emma Satish for all their love and support.

My lovely parents raised me and trained me to be a happy, independent and brave girl to start my own life. I can never forget the sacrifices they have made for me, especially during their most difficult times. They loved me, trained me and supported me throughout my life and encouraged me to embrace life with an optimistic attitude, no matter life is easy or tough and no matter I succeed or lose.

Satish and Emma are two important people in my life. They joined my journey half way and will accompany me till the end of this adventure. We have shared all the joys and tears together. We have witnessed and will witness all the minor or major events in our life and will support each other forever. I love you both. 


\section{ACKNOWLEDGEMENTS}

I would like to thank my mentor, Dr. Brian Sorrentino for giving me the opportunity to work with him. His guidance and support has been invaluable. Graduate training under his mentorship was a great experience and has prepared me for my future endeavors.

I also want to thank my lab members for sharing with me their experience and advise in and out the lab. I specially would like to thank Jie Jiang, former graduate student in our lab. She helped me start my project and trained me to be independent student before she left the lab. Even now we still exchange ideas about life and work.

I would also like to thank my committee members, Dr. Suzanne Baker, Dr. Janet Partridge, Dr. Wing Leung and Dr. Lawrence Pfeffer for their guidance during graduate training. Their suggestions and corrections helped shape my project which finally gets published.

I would like to acknowledge the Flow Cytometry and Cell Sorting core facility and Animal Resource Center in St. Jude Children's Research Hospital. Without their professional assistance with cell sorting and animal care this project can't be done. 


\begin{abstract}
Overexpression of HOXB4 in hematopoietic stem cells (HSCs) leads to increased self-renewal without causing hematopoietic malignancies in transplanted mice. The molecular basis of HOXB4-mediated benign HSC expansion in vivo is not well understood. To gain further insight into the molecular events underlying HOXB4mediated HSC expansion, we analyzed gene expression changes at multiple time points in $\mathrm{Lin}^{-} \mathrm{Scal}^{+} \mathrm{c}-\mathrm{kit}^{+}$(LSK) cells from mice transplanted with bone marrow (BM) cells transduced with a MSCV-HOXB4-ires-YFP vector. A distinct HOXB4 transcriptional program was reproducibly induced and stabilized by 12 weeks after transplant. Dynamic expression changes were observed in genes critical for HSC self-renewal as well as genes involved in myeloid and B cell differentiation. Prdm16, a transcription factor associated with human acute myeloid leukemia (AML), was markedly repressed by HOXB4 but upregulated by HOXA9 and HOXA10, suggesting that Prdm16 downregulation was involved in preventing leukemia in HOXB4 transplanted mice. Functional evidence to support this mechanism was obtained by enforcing co-expression of sPrdm16 and HOXB4, which led to myeloid expansion, and leukemia. Before the onset of leukemia decreased HSC frequency was observed in HOXB4and sPrdm16 co-expressing cells, suggesting sustained expression of Prdm16 abolished HOXB4-mediated HSC expansion. Altogether, these studies define the transcriptional pathways involved in HOXB4 HSC expansion in vivo and identify repression of Prdm16 transcription as a mechanism by which HOXB4 mediates a benign HSC expansion.
\end{abstract}




\section{TABLE OF CONTENTS}

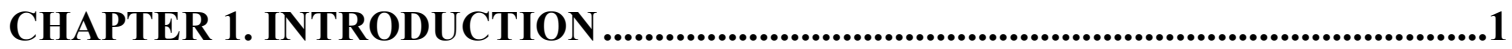

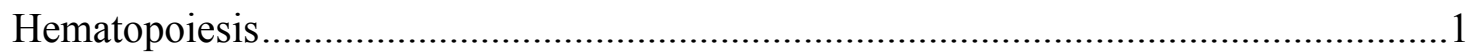

Adult hematopoietic hierarchy.......................................................................

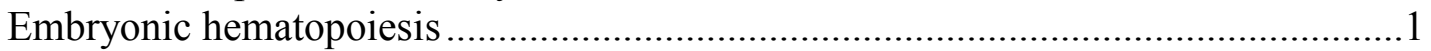

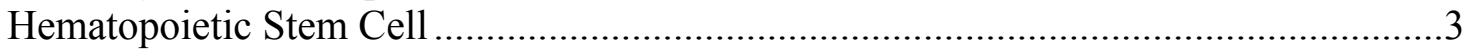

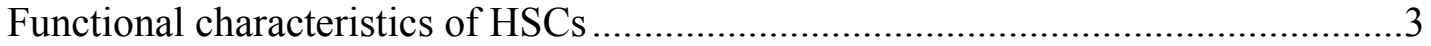

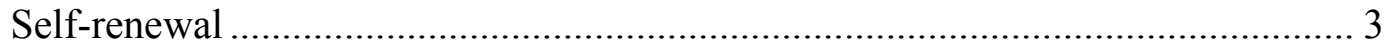

Multilineage differentiation potential ............................................................ 5

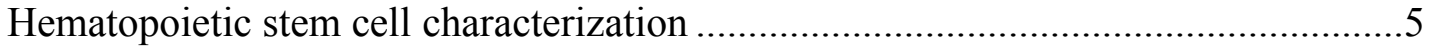

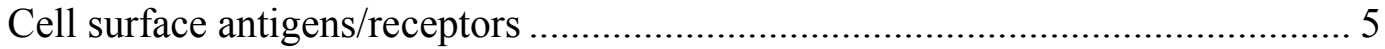

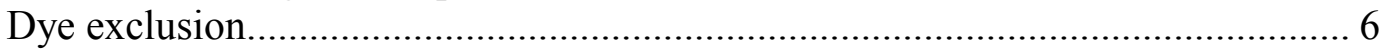

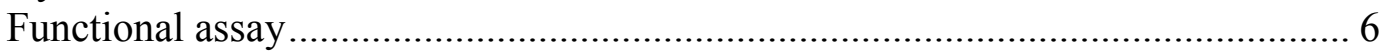

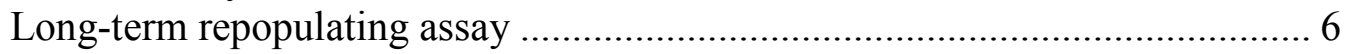

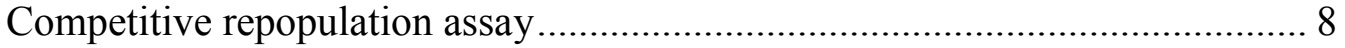

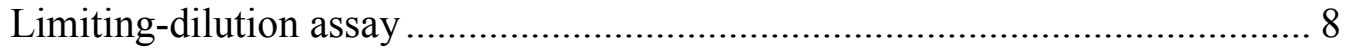

Serial transplantation assay ................................................................. 8

Regulation of Hematopoietic Stem Cell Pool.............................................................

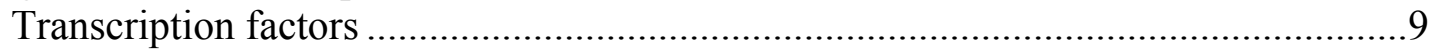

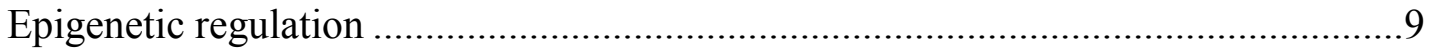

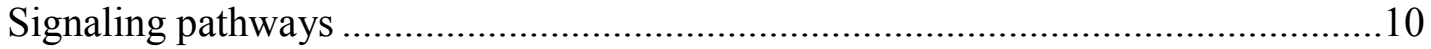

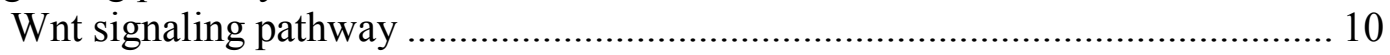

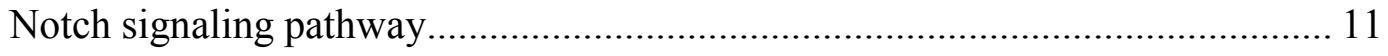

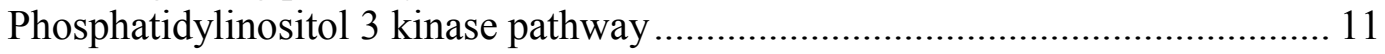

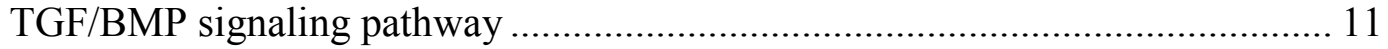

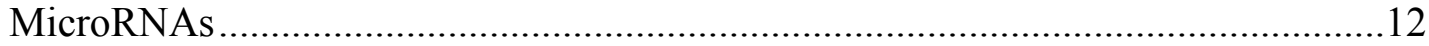

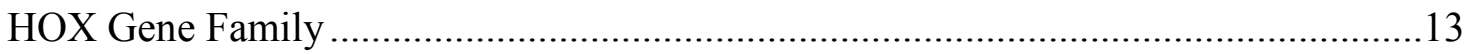

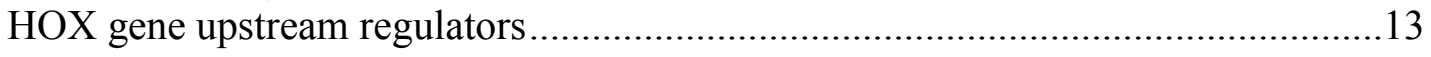

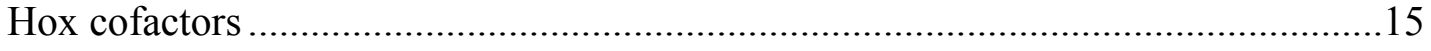

Role of Hox genes in normal hematopoiesis ................................................. 15

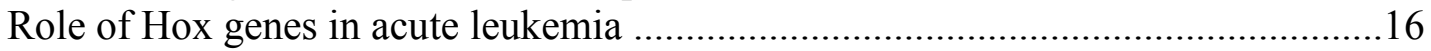

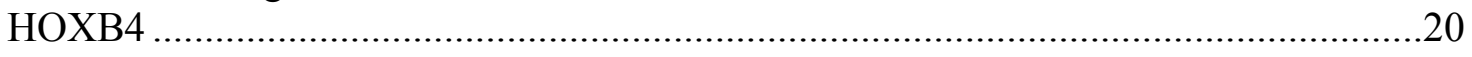

HOXB4 role in embryonic stem cell derived hematopoiesis................................20

HOXB4 overexpression enhances adult HSC self-renewal ..................................20

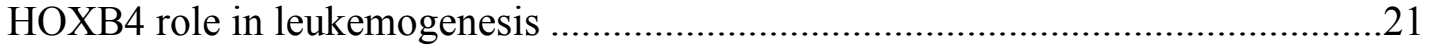

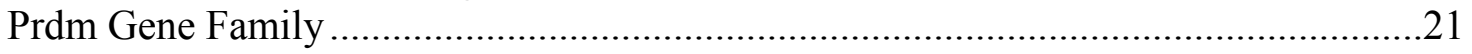

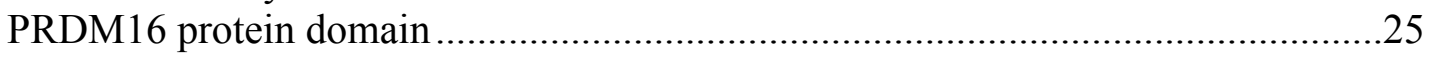

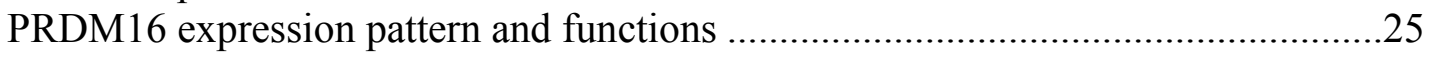

PRDM16's role in normal hematopoiesis ......................................................... 26

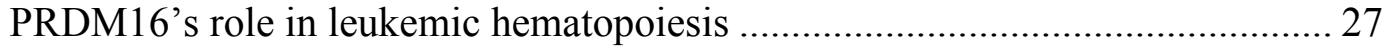

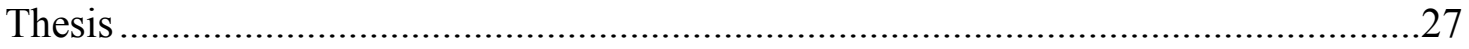

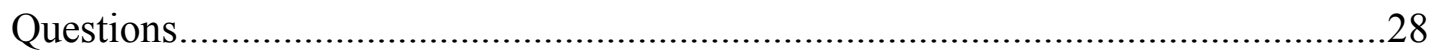

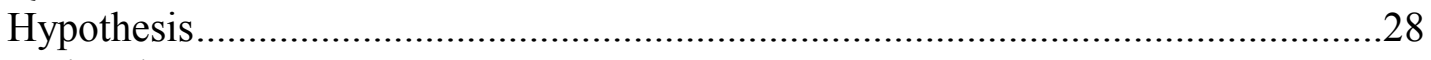

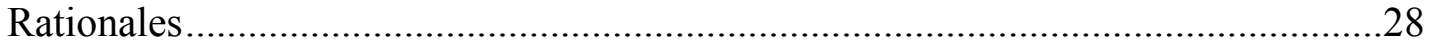




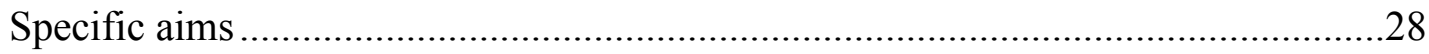

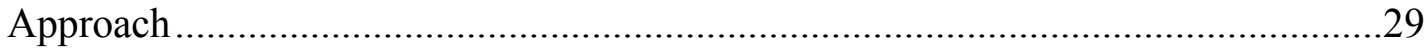

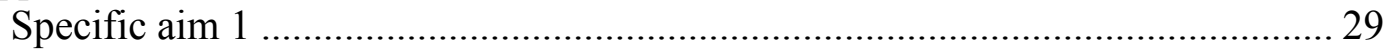

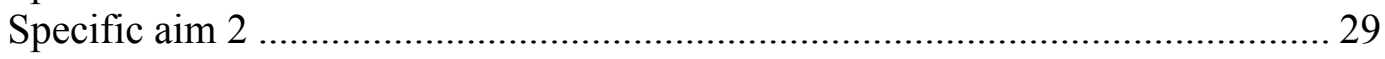

\section{CHAPTER 2. IDENTIFICATION OF HOXB4 TRANSCRIPTIONAL} TARGET GENES IN PRIMITIVE HEMATOPOIETIC CELLS IN VIVO ..............30

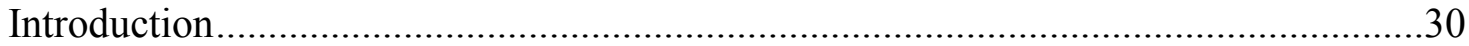

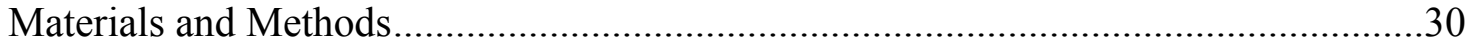

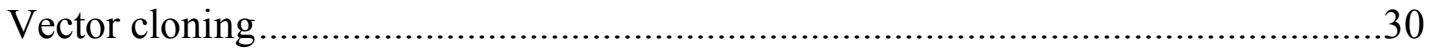

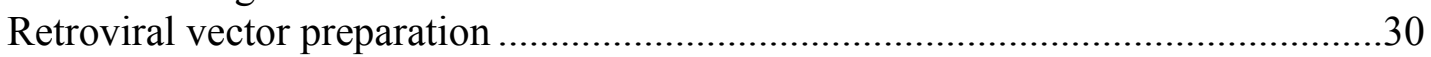

Bone marrow cells isolation, culture, transduction and transplantation .....................31

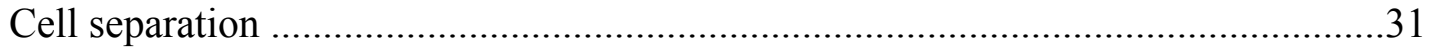

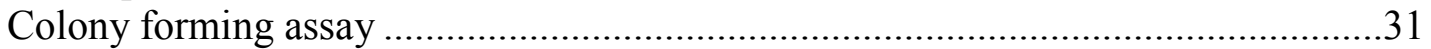

Microarray expression analyses and real-time RT-PCR ...........................................32

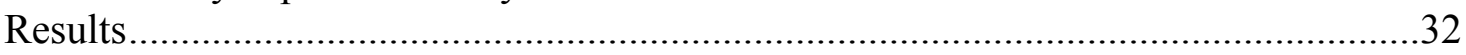

HOXB4 ${ }^{+}$LSK cells demonstrated enhanced hematopoietic progenitor cell self-

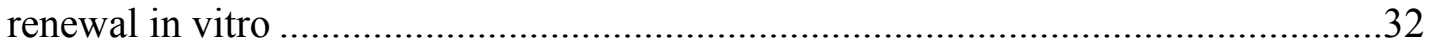

Global gene expression changes in HOXB4-transduced LSK cells at various

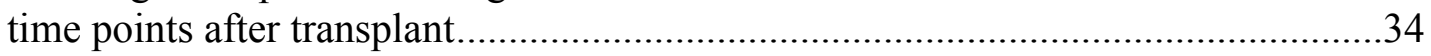
Identification of specific changes in gene expression in HOXB4-transduced LSK

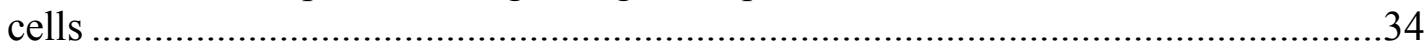

HOXB4 overexpression results in gene expression changes associated with HSC self-renewal and lymphoid-myeloid differentiation ....................................................39

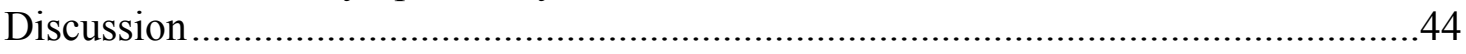

Advantage of gene profiling study in LSK cells from HOXB4 transplanted mice ...44 Mechanism of well-maintained HSC pool size by HOXB4 ........................................44

Mechanism how HOXB4 prevents leukemia in transplanted mice ............................45

\section{CHAPTER 3. DOWNREGULATION OF PRDM16 PREVENTS LEUKEMIA} IN HOXB4 TRANSPLANTED MICE................................................................48

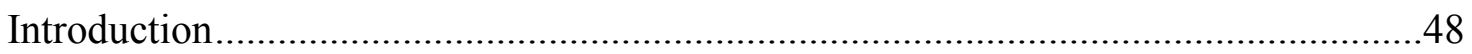

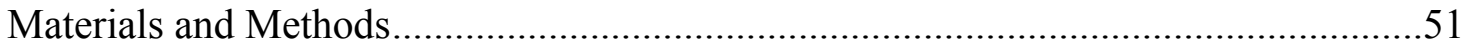

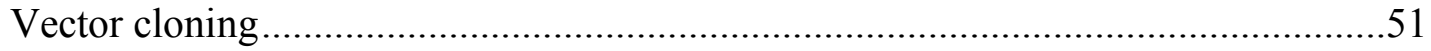

Retroviral vector preparation .................................................................................51

Calcium phosphate transfection and vector concentration for lentiviral vector .........52

Bone marrow cells isolation, culture and transduction.............................................52

In vitro expansion assay ................................................................................52

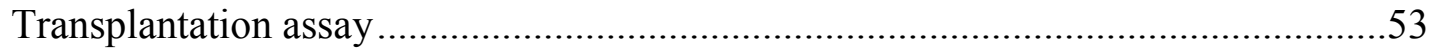

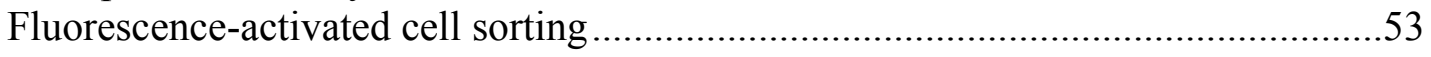

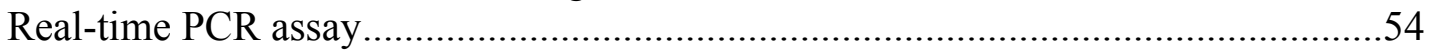

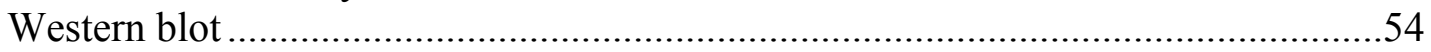

Immunofluorescence staining and confocal imaging...............................................54

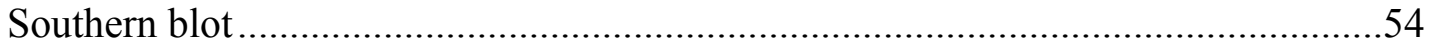

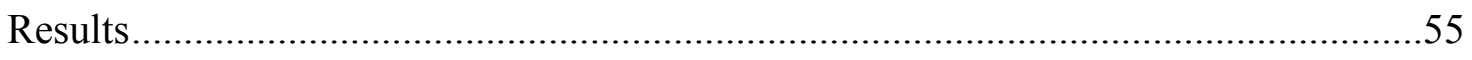

Endogenous Prdm16 expression in LT-HSC and ST-HSCs.....................................55 
Overexpression of HOXA9 and HOXA10 led to growth advantage and enhanced

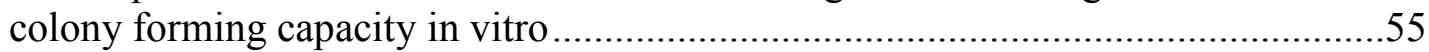

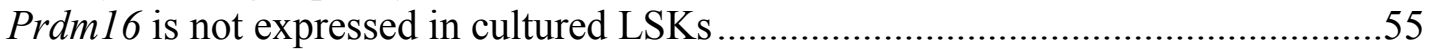

Prdm16 downregulation is restricted to HOXB4-expressing HSCs in vivo..............58

Lentiviral sPrdm 16 construct and sPRDM16 protein expression............................58

Low transduction efficiency of sPrdm16 overexpression vector ..............................58

Increased colony forming capacity of HOXB4 and sPRDM16 coexpressing cells...64

Enforced co-expression of sPRDM16 and HOXB4 causes preleukemia in mice .....64

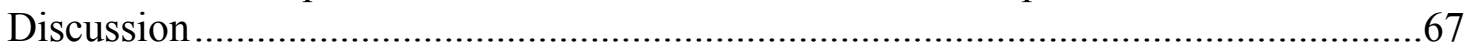

\section{CHAPTER 4. DOWNREGULATION OF PRDM16 IS REQUIRED FOR

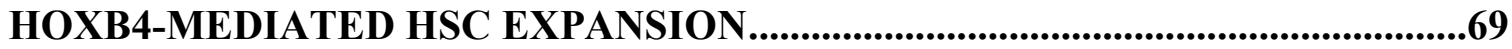

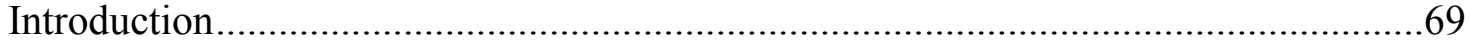

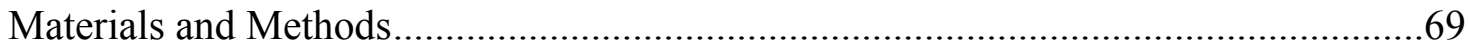

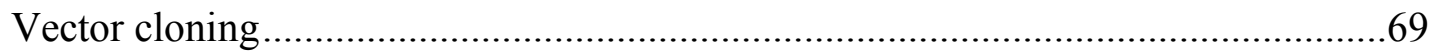

Calcium phosphate transfection and vector concentration for lentiviral vector ........70

sPRDM16 protein induction in vitro................................................................ 70

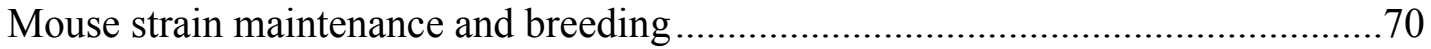

Polymerase chain reaction (PCR) genotyping .................................................... 70

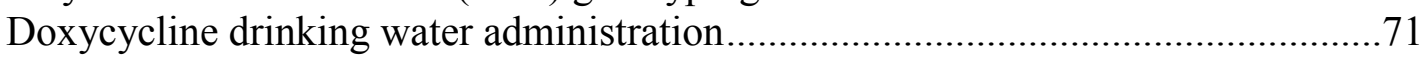

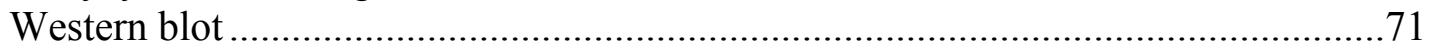

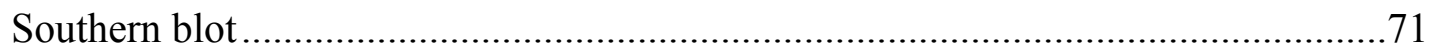

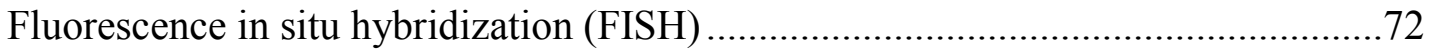

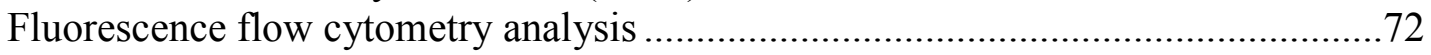

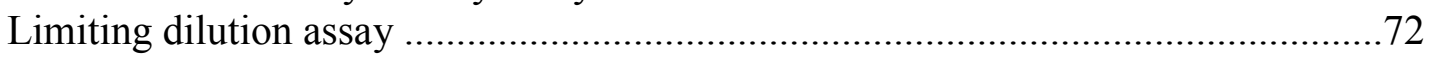

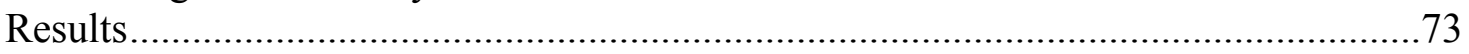

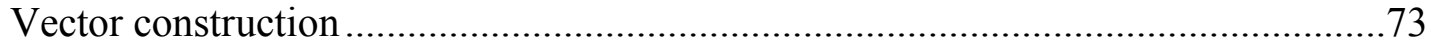

Dose-dependent induction of sPRDM16 and GFP protein................................73

Generation and validation of TetO-sPrdm16 transgenic mice..................................73

Overexpression of $s$ Prdm 16 abolishes HOXB4-mediated HSC expansion...............82

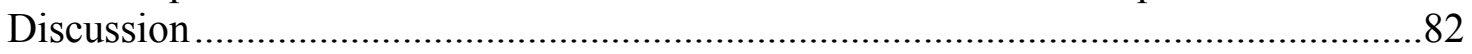

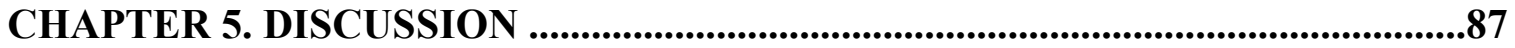

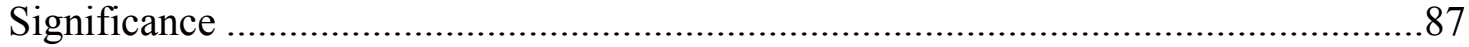

HOXB4 is a global regulator of HSC self-renewal .............................................87

Differential regulation of Prdm16 transcript by HOXB4 and HOXA9/A10 .............87

HOXB4 doesn't cause leukemia unless pro-oncogenes are activated .......................89

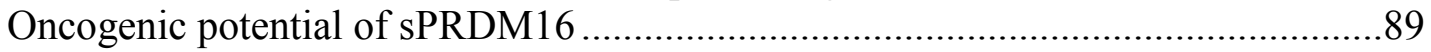

MDS/AML genetic lesion is missing from HOXB4/sPrdm16 preleukemia model ..90 Repression of Prdm16 may promote cell enter cell cycle and facilitate HOXB4-

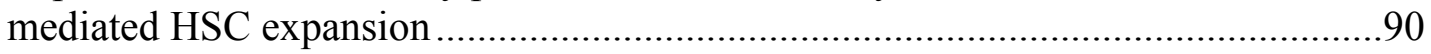

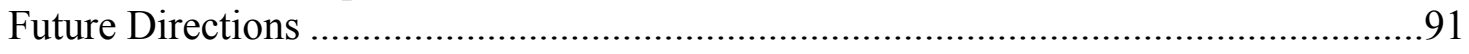

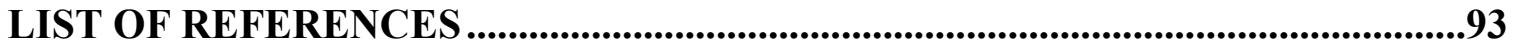

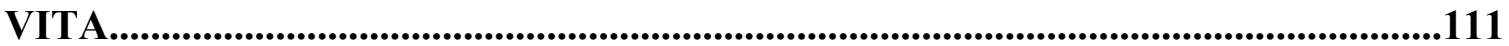




\section{LIST OF TABLES}

Table 1-1. Phenotype of murine hematopoietic stem cell at different development

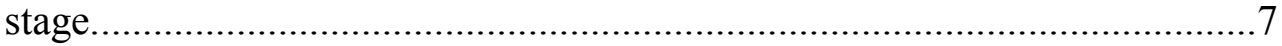

Table 1-2. HOX gene studies. ..................................................................................

Table 2-1. Summary of RNA samples submitted for microarray from GFP or HOXB4 group at 6 weeks, 12 weeks and 20 weeks. ....................................36

Table 2-2. Pathways activated in HOXB4-LSKs at 20 weeks.........................................41

Table 2-3. Pathways activated in GFP-LSKs at 20 weeks...........................................42

Table 4-1. HSC frequency in HOXB4 and sPRDM16 transplanted mice. .....................84 


\section{LIST OF FIGURES}

Figure 1-1. The hierarchy of hematopoietic cells.................................................

Figure 1-2. Timeline of hematopoietic events in the mouse conceptus. ........................4

Figure 1-3. A schematic structure of clustered HOX genes. .......................................14

Figure 1-4. The domain structure of the mouse PRDM1-16 proteins. ...........................22

Figure 1-5. Structural and regulatory features of PRDM16...................................23

Figure 2-1. Secondary colony numbers generated from $1 \times 10^{4}$ GFP-LSK cells or HOXB4-LSK cells at 12 or 20 weeks post transplantation. ......................33

Figure 2-2. Experimental design of HOXB4 target gene analysis in transplanted murine HSCs.

Figure 2-3. PCA and heat map representation of HOXB4-induced expression profiles in bone marrow LSK cells. ........................................................37

Figure 2-4. Identification and validation of HOXB4 target genes. ..............................38

Figure 2-5. GSEA and MetaCore pathway analysis of HOXB4 responsive genes........40

Figure 2-6. Activation of B cell differentiation and Granulocyte development/ GCSF-induced myeloid differentiation in 12wk and 20wk HOXB4-LSKs. ..43

Figure 2-7. Regulation of HSC pool size in HOXB4 transplanted mice........................46

Figure 3-1. HOXB4-mediated leukemia in transplanted mice.

Figure 3-2. Vector insertion site-mediated oncogene activation in HOXB4-mediated leukemia 2 in transplanted mice.

Figure 3-3. Quantitative real-time PCR of endogenous Prdm16 mRNA levels in various sorted progenitor and stem cell populations.

Figure 3-4. Growth advantage and enhanced myeloid progenitor self-renewal observed in HOXB4, HOXA9 and HOXA10 transduced cells in vitro

Figure 3-5. Relative Prdm16 expression in HOXB4, HOXA9 and HOXA10 transduced LSKs in vitro.

Figure 3-6. Downregluation of Prdm16 is restricted in HOXB4 expressing HSCs in vivo.

Figure 3-7. Lentiviral vectors engineered to overexpress sPrdm16 and HOXB4. 
Figure 3-8. Low transduction of sPrdm16 vector in murine bone marrow cells.

Figure 3-9. Depletion of sPRDMdfdf16 expressing cells was not due to vector rearrangement.

Figure 3-10. Secondary colony numbers by myeloid CFU-C assay on sorted transduced cells as a measure of self-renewal capacity.

Figure 3-11. Enforced co-expression of HOXB4 and sPrdm16 enhances HPC selfrenewal in vitro and induces myeloid pre-leukemic transformation in transplanted mice.

Figure 4-1. Dose-dependent induction of sPRDM16 and GFP protein with TetOn inducible system.

Figure 4-2. Schematic diagram of TetO-sPrdm16 transgenic mice generation. 75

Figure 4-3. TetO-sPrdm16 transgenic mice PCR genotyping. .................................. 76

Figure 4-4. GFP expression in peripheral blood of N1 progenies...............................78

Figure 4-5. Southern blot of TetO-sPrdm16 transgenic mice.

Figure 4-6. TetO-sPrdm16 transgene integration site by FISH and protein expression in transgenic bone marrow cells.

Figure 4-7. GFP percentage in HSC, progenitor and mature lineages in transgenic bone marrow 39 days post doxycycline induction.

Figure 4-8. MPP and HSC frequency on marking cells.

Figure 5-1. Comparison of in vitro and in vivo Hox-binding site preferences. .88 


\section{LIST OF ABBREVIATIONS}

AG

AGM

ALL

AML

$\mathrm{B} 1 \mathrm{H}$

B-ALL

BAT

BM

BMP

$\mathrm{CaCl}_{2}$

CAFC

CFC-E

CFC-GEMM

CFU-C

CFU-S

ChIP

CLL

CLP

CMP

CPR

CRU

CtBP

Dnmt

DRG

DsRNA

EB

ECM

ECs

EHMT1

EMSA

ESC

ETPs

EVI1

FACP

FACS

FBS

FISH

G-CSF

GDF

GFP

GMP

GSEA
Adrenal gland

Aorta-gonad-mesonephros

acute lymphocytic/lymphoid leukemia

Acute myelogenous leukemia

Bacterial one-hybrid

B-cell acute lymphocytic/lymphoid leukemia

Brown adipose tissue

Bone marrow

Bone morphogenetic protein

Calcium chloride

cobblestone area-forming cell assay

Erythroid colony-forming cells

Granulocyte/erythroid/macrophage/megakaryocyte colony-forming cells

Colony forming unite-culture

Colony forming unit-spleen

Chromatin immunoprecipitation

Chronic lymphocytic leukemia

Common lymphoid progenitor

Common myeloid progenitor

Cartilage primordial of the ribs

Competitive reconstitution unit

C-terminal binding protein

DNA methyltransferase

Dorsal root ganglia

Double-stranded RNA

Embryonic body

Extracellular matrix

Endothelia cells

Euchromatic histone-lysine-methyltransferase 1

Electrophoretic mobility shift assay

Embryonic stem cell

Earliest thymic progenitors

Ecotropic viral integration site 1

Facio-acoustic preganlion complex

Fluorescence-activated cell sorting

Fetal bovine serum

Fluorescence in situ hybridization

Granulocyte-colony stimulating factor

Growth and differentiation factor

Green fluorescence protein

Granulocyte-monocyte/macrophage progenitor

Gene set enrichment analysis 


\begin{tabular}{|c|c|}
\hline $\mathrm{H} 3 \mathrm{~K} 4 \mathrm{me} 3$ & Tri-methylation of lysine 4 of histone $\mathrm{H} 3$ \\
\hline H3K9me2 & Di-methylation of lysine 9 of histone $\mathrm{H} 3$ \\
\hline HATs & Acetyltransferase \\
\hline HDACs & Histone deacetylase \\
\hline HMT & Histone lysine methyltransferase \\
\hline HPC & Hematopoietic progenitor cell \\
\hline HSC & Hematopoietic stem cell \\
\hline HSPC & Hematopoietic stem progenitor cell \\
\hline I-Smad & Inhibitory Smad \\
\hline IRES & Internal ribosome entry site \\
\hline LB & Limb bud \\
\hline LICs & Leukemia-initiating cells \\
\hline $\operatorname{Lin}^{-}$ & Lineage marker negative \\
\hline LSCs & Leukemia stem cells \\
\hline Lsd1 & lysine-specific demethylase 1 \\
\hline LSK & Lineage ${ }^{-S c a}-1^{+} \mathrm{c}-\mathrm{kit}^{+}$ \\
\hline LT-HSC & Long-term hematopoietic stem cell \\
\hline LTC-ICs & Long-term culture-initiating cells \\
\hline $\mathrm{MC}$ & Meckel's cartilage \\
\hline Mdr & Multidrug resistance protein \\
\hline MDS & Myelodysplastic syndrome \\
\hline MEIS & Myeloid ectopic insertion site \\
\hline MEL & Murine erythroid leukemia \\
\hline MEL1 & MDS1/EVI1 like gene 1 \\
\hline MEP & Megakarocyte-erythroid progenitor \\
\hline MIS & Muellerian inhibiting substance \\
\hline MLL & Mixed-lineage leukemia \\
\hline MPPs & multipotent hematopoietic progenitors \\
\hline NOD/SCID & Nonobese diabetic-severe combined immunodeficiency \\
\hline NPM1 & Nucleophosmin 1 \\
\hline NS & Nasal spetum \\
\hline NSG & NOD/SCID/IL2R- $\gamma^{\text {null }}$ \\
\hline $\mathrm{OE}$ & Oral epithelium \\
\hline OS & Optic stalk \\
\hline PB & Peripheral blood \\
\hline PBMs & Protein-binding microarrays \\
\hline PBX & Pre-B-cell leukemia \\
\hline PCA & Principle component analysis \\
\hline PcG & Polycomb-group \\
\hline PCR & Polymerase chain reaction \\
\hline PGC-1 $\alpha$ & PPAR- $\gamma$-coactivator- $1 \alpha$ \\
\hline PGC- $1 \beta$ & PPAR- $\gamma$-coactivator- $1 \beta$ \\
\hline PGCs & Pluripotent primordial germ cells \\
\hline PI-3 kinase & Phosphatidylinositol 3-kinase \\
\hline PIP3 & Phosphatidylinositol-3,4,5-trisphosphate \\
\hline Prdm & PRDI-BF1 and RIZ homology domain containing \\
\hline
\end{tabular}




$\begin{array}{ll}\text { Pre-miRNA } & \text { Pre-microRNA } \\ \text { Prmt5 } & \text { Protein methyltransferase 5 } \\ \text { R-Smad } & \text { Receptor-regulated Smad } \\ \text { RD } & \text { Repressor domain } \\ \text { Rh123 } & \text { Rhodamine123 } \\ \text { RRE } & \text { Rev response element } \\ \text { RT } & \text { Room temperature } \\ \text { SCF } & \text { Stem cell factor } \\ \text { SHH } & \text { Sonic hedgehog } \\ \text { SP } & \text { Side population } \\ \text { sPrdm16 } & \text { Short form of Prdm16 } \\ \text { ST-HSC } & \text { Short-term hematopoietic stem cell } \\ \text { T-ALL } & \text { T-cell acute lymphocytic/lymphoid leukemia } \\ \text { TALE } & \text { Three animo acid loop extension } \\ \text { TSS } & \text { Transcription start site } \\ \text { TV } & \text { Telencephalic vesicle } \\ \text { WAT } & \text { White adipose tissue } \\ \text { WT } & \text { Wild type } \\ \text { 5-FU } & \text { 5-Fluorouracil }\end{array}$




\section{CHAPTER 1. INTRODUCTION}

\section{Hematopoiesis}

All the blood cells are continuously generated by hematopoietic stem cells (HSCs) throughout life. Maintenance of hematopoietic system homeostasis and replenishment of mature blood cells rely on the self-renewal capacity and multipotency of HSCs. Hematopoiesis includes adult and embryonic hematopoiesis, which arises from different hematopoietic organs and undergoes distinct biology process. So far the murine hematopoietic system is the best understood of all species.

\section{Adult hematopoietic hierarchy}

Hematopoiesis is the process of production, multiplication and specification of blood cells in the bone marrow. With a combination of cell surface marker expression and functional assays, the hierarchical structure of the hematopoietic system was achieved (Figure 1-1). HSCs are the first well-characterized tissue-specific stem cells with self-renewal and multipotency and reside at the top of the hierarchy. HSCs give rise to the multipotent progenitor (MPP) ${ }^{1,2}$ which further differentiated into two oligopotent progenitors: the common lymphoid progenitor (CLP) ${ }^{3}$ and the common myeloid progenitor (CMP). ${ }^{4} \mathrm{CMPs}$ then give rise to megakaryocyte-erythrocyte progenitors (MEPs) and granulocyte-macrophage progenitors (GMPs). These downstream oligopotent progenitors then give rise to all of lineage-committed effector cells and downstream mature blood cells.

However, recent studies suggested that alternative models for lineage commitment during hematopoiesis should be considered. In 2008 Bhandoola's gourp demonstrated that the earliest thymic progenitors (ETPs) are not derived from lymphoidrestricted progenitors but from multipoient bone marrow progenitors, which lose $\mathrm{B}$ potential either prethymically or intrathymically. ${ }^{5}$ Almost all ETPs derived from multipotent progenitors possess both $\mathrm{T}$ and myeloid potential within the thymus. ${ }^{5}$ Later on Stuart H. Orkin's group proposed an alternative mouse hematopoietic hierarchy from single-cell expression data which indicated that the MegE lineage priming occurred within highly enriched HSCs. ${ }^{6}$ This is different from the model proposed by Jorden Adolfsson et al and Cornelis J.H. Pronk et al which placed MegE lineage downstream of MPP and CMP.,

\section{Embryonic hematopoiesis}

The first wave of hematopoiesis starts in the extraembryonic yolk sac at E7.5 and later in allantois and placenta. However functional experiment showed that hematopoietic cells generated in yolk sac prior to E9.5 lack colony forming unit-spleen (CFU-S) ${ }^{9}$ and

lack definitive HSCs prior to E11.5. ${ }^{10,11}$ The aorta-gonad-mesonephros (AGM) region of 


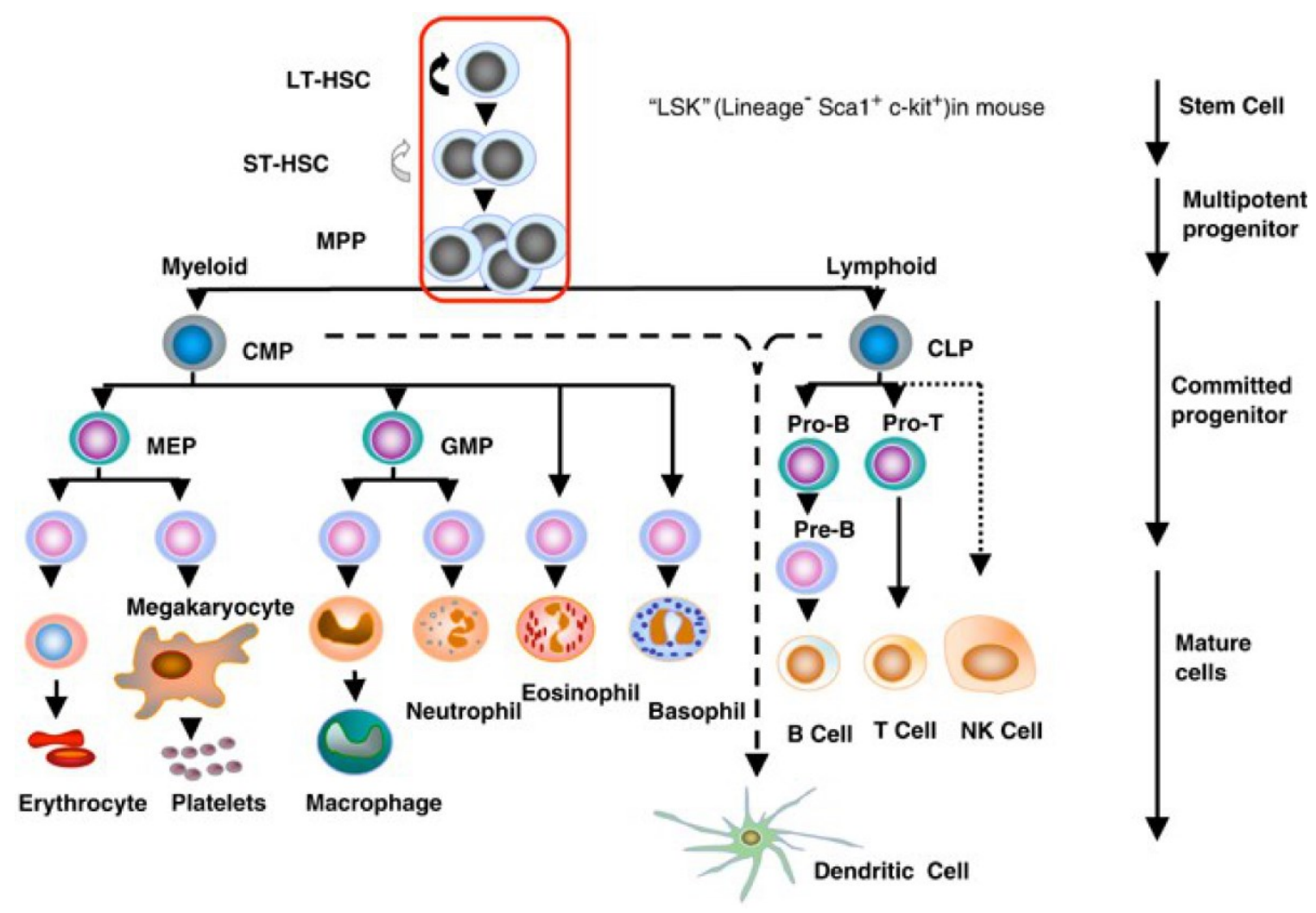

Figure 1-1. The hierarchy of hematopoietic cells.

LT-HSC, long-term repopulating HSC; ST-HSC, short-term repopulating HSC; MPP, multipotent progenitor; CMP, common myeloid progenitor; CLP, common lymphoid progenitor; MEP, megakaryocyte/erythroid progenitor; GMP, granulocyte-macrophage progenitor. The encircled pluripotent population, LT-HSC, ST-HSC and MPP are Lin-, Sca-1+, c-kit+ as shown.

Reprinted with permission. Larsson J, Karlsson S. The role of Smad signaling in hematopoiesis. Oncogene. 2005;24(37):5676-5692. ${ }^{12}$ 
the mouse embryo starts to make blood cells from E9.5 and was proven to be a powerful source of definitive HSCs. ${ }^{10,11}$ From E12.5 hematopoiesis switches to fetal liver and HSCs undergo dramatic expansion. Right before birth bone marrow becomes the major site of hematopoiesis through the whole life. (Figure 1-2)

\section{Hematopoietic Stem Cell}

HSCs are rare population and occur at a frequency of 1 stem cell in 10,000 to $1,000,000$ bone marrow cells. HSCs are derived from mesoderm and give rise to all the downstream progenitor cells and mature blood cells. Hemogenic endothelium hypothesis proposes that HSCs arise directly from committed endothelia cells (ECs) of the ventral aspect of the dorsal aorta in the $\mathrm{AGM}^{13,14}$ which is distinct from the hemangioblast hypothesis which supposes that a common bipotent precursor gives rise to both vasculature and the blood cells. Initial understanding of HSCs started with the discovery of CFU-S described by Till and McCulloch. ${ }^{15}$ They demonstrated that intravenously injected bone marrow cells were capable of forming distinct hematopoietic colonies in the spleen of irradiated mice, and these colonies contained erythrocytes, megakaryocytes an granulocytes/macrophages. ${ }^{15}$ Later studies pointed out there are at least two type of CFU-S: colonies that formed at day 8 after transplantation and those formed at day $12 .^{16,17}$ They are referred as day 8-CFU-S and day12-CFU-S. Cells residing in day8CFU-S were more committed progenitors with little or no self-renewal capacity and cells in day12-CFU-S contained certain self-renewing cells. ${ }^{18}$ Morrison and Weissman further confirmed that LT-HSC and ST-HSC can only be purified from day-12 to day-14 CFU$\mathrm{S}^{1}$.

\section{Functional characteristics of HSCs}

The functional characteristics of HCSs are self-renewal and multilineage differentiation potential.

\section{Self-renewal}

Self-renewal of HSCs is critical for HSC pool maintenance to prevent the exhaustion of blood formation. It is usually achieved by either symmetric or asymmetric cell division or both. Symmetric division will result in two identical daughter stem cells or two lineage-restricted progenitors while asymmetric division will generate one daughter stem cell and one differentiated cell ${ }^{19}$. Asymmetric cell division doesn't generate net increase of HSC numbers. Self-renewal potential of HSCs is associated with the activity of telomerase $\mathrm{e}^{20-22}$. Telemetric shortening during cell division usually results in cell cycle arrest, chromosomal instability and replicative senescence and causes the loss of self-renewal capacity of HSCs which directly reduces HSC pool size. 


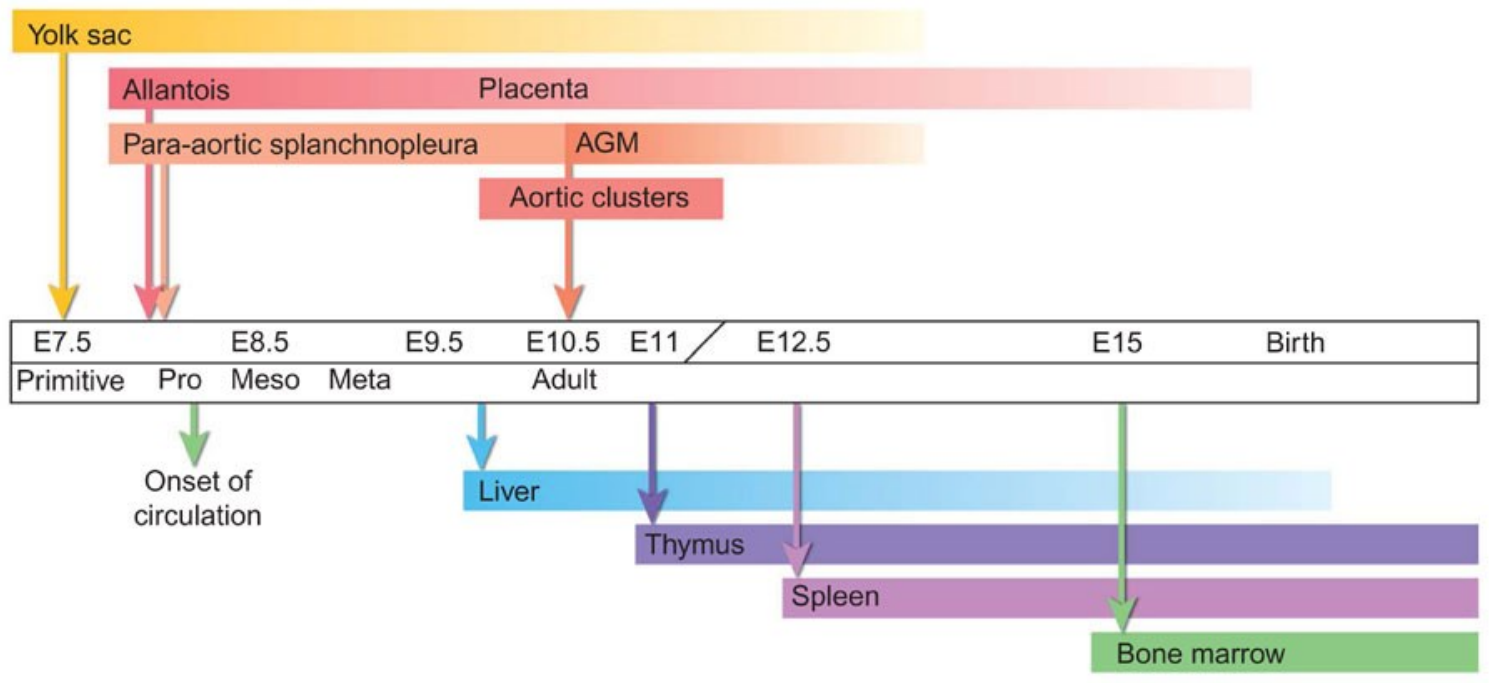

Figure 1-2. Timeline of hematopoietic events in the mouse conceptus.

Arrows above indicate the onset of specific hematopoietic cell generation and/or appearance; arrows below indicate the earliest time of colonization of the secondary hematopoietic territories. AGM, Aorto Gonado Mesonephros region.

Reprinted with permission: Dzierzak E, Speck NA. Of lineage and legacy: the development of mammalian hematopoietic stem cells. Nat Immunol. 2008;9(2):129$136 .^{23}$ 


\section{Multilineage differentiation potential}

Multilineage differentiation means HSCs are able to produce progeny that contribute to all blood cell lineages. ${ }^{24-30}$ The decision of HSCs to self-renew or differentiation may be random, but the external signals from bone marrow niche play role in the cell fate decision of HSCs during lineage commitment through cell-cell or cellextracellular matrix (ECM) interactions or through cytokines, chemokines or other extracellular molecules. Signaling pathways present in bone marrow niche, such as Wnt pathway and Notch pathway, act on HSCs or downstream progenitors to regulate HSC lineage commitment. Recent evidence showed multilineage priming is one mechanism how HSCs maintain multilineage commitment potential without changing global gene expression. Open chromatin structure of HSCs or multipotent progenitors leaves many key cis-acting regulatory elements of lineage-affiliated genes accessible which allows these genes to be available for transcription. Transcription factors which favor different lineage commitment may negatively interact with each other resulting in the dominant upregulation of a given program with the shutdown of other program consistent with dominant lineage selected.

\section{Hematopoietic stem cell characterization}

Murine HSCs are a rare population capable of reconstituting recipients and reside in fetal liver and adult BM. There are studies to characterize and purify hematopoietic stem cells for decades. It becomes clear that combination of surface markers/dye exclusion and in vivo/ex vivo functional assays is necessary to identify functional HSCs which are able to reconstitute lethally irradiated hosts. More defined subpopulations with distinct reconstitution capacity were identified with combination of newly discovered cell surface markers by many groups. Here we focus on the phonotypical and functional characterization of murine hematopoietic stem cells.

\section{Cell surface antigens/receptors}

In 1988 Weissman's group first identified multilineage HSC as a highly enriched rare population in lineage marker negative $\left(\operatorname{Lin}^{-}\right), \mathrm{Sca}^{+}{ }^{+}$and thy $1.1^{\text {low }}$ cells, which represent about $0.05 \%$ of the whole bone marrow. ${ }^{27}$ Ikuta, $\mathrm{K}$ et al ${ }^{31}$ showed that both HSCs and progenitors express Kit surface marker, but only Lin ${ }^{-} \mathrm{c}-\mathrm{Kit}^{+} \mathrm{Sca}-1^{+}$cells are primitive HSCs and response better to SCF and stromal cells. ${ }^{32}$ The flk-2 gene, a tyrosine kinase receptor, was shown not to be expressed in FR25 Lin ${ }^{-}-\mathrm{kt}^{\mathrm{BR}}$ population which is highly enriched for $\mathrm{HSCs}^{33}$ and flk-2 was characterized as HSC marker to further purify LT-HSC from adult bone marrow cells as c-Kit ${ }^{+} \mathrm{Lin}^{-} \mathrm{Sca}-1^{+} \mathrm{Flk}-2^{-}$cells from mice. ${ }^{2} \mathrm{Flk}-2$ can only be used to separate adult HSCs as fetal liver HSCs express Flk-2. CD34 is a human HSC marker. ${ }^{34}$ Unlike human HSCs, mouse long-term multilineage HSCs are present in $\mathrm{CD} 34{ }^{\mathrm{lo} /}{ }^{-} \mathrm{c}-\mathrm{Kit}^{+} \mathrm{Sca}-1^{+} \mathrm{Lin}^{-}$fraction, ${ }^{30}$ providing a new marker to separate mouse HSCs at a near homogenous level, even though only $20 \%$ of intravenously injected $\mathrm{CD} 34^{\mathrm{lo} /}{ }^{\mathrm{c}-\mathrm{Kit}}{ }^{+} \mathrm{Sca}-1^{+} \mathrm{Lin}^{-}$cells conferred long-term multilineage reconstitution. ${ }^{30}$ 
However, in fetuses and neonates CD34 is also expressed in long-term repopulating HCSs. ${ }^{35}$ So using CD34-LSK as a marker to purify HSCs is only suitable for mice older than 10 weeks due to age-dependent CD34 expression in HSCs. More recently combination of SLAM family member CD150 and CD48 with Lineage marker, Sca-1 and c-Kit was found to be able to purify more enriched functional long-term HCSs. ${ }^{36}$ One out every $2.1 \mathrm{CD} 50^{+} \mathrm{CD} 48^{-} \mathrm{Sca}-1^{+} \mathrm{Lin}^{-} \mathrm{c}-\mathrm{Kit}^{+}$cells gave long-term multilineage reconstitution in lethally irradiated mice. ${ }^{36}$

\section{Dye exclusion}

Hoechst $33342^{\text {low }}$ side population (SP) is a small subset of population of murine bone marrow cells which is able to block the Hoechst efflux activity due to a multidrug resistance protein (mdr) or mdr-like mediated efflux. SP was shown to be enriched about 1000 fold HSC activity and Hoechst 33342 was used to isolate quiescent and replicating HSCs. ${ }^{37,38}$ Similar to Hoechst33342, Rhodamine123 (Rh123) is another vital dye used to label relative more quiescent murine HSCs and Rh123 ${ }^{\text {low }}$ cells self-renew to a greater degree than Rh123 high population. ${ }^{39} \mathrm{Rh} 123^{\text {low }}$ and Hoechst $33342^{\text {low }}$ population both represent G0/G1 HSCs with higher level of self-renewal and long-term multilineage reconstitution capacity, which were lost within S/G2/M subpopulation. ${ }^{39}$ Resistance to active DNA replication provided these quiescent HSCs the radioprotection activity.

The phenotype of murine hematopoietic stem cells at different development stages is summarized in Table 1-1.

\section{Functional assay}

In vitro functional assays developed for HSCs are cobblestone area-forming cell (CAFC) assay and long-term culture-initiating cell (LTC-IC) assay. Actually they are not able to identify true HSCs; instead they are the assays identifying relative immature cells in culture. The first in vivo assay to assess HSC function is thought to be CFU-S ${ }^{15}$ until the cells forming spleen colonies were proven to be hematopoietic progenitors with shortterm potential. ${ }^{18}$ To test repopulating HSC function long-term transplantation assay is required.

\section{Long-term repopulating assay}

Long-term repopulating assay requires intravenous injection of test bone marrow cells to irradiated or compromised hosts. Test cells and host cells should be distinguishable from each other by different alleles of the hematopoietic cell marker CD45.1 or CD45.2. In earlier studies cells were distinguished by different hemoglobin ${ }^{40}$ or gender. ${ }^{41}$ Peripheral blood (PB) is collected at various intervals and contribution of test cells to myeloid, T lymphoid and B lymphoid lineage are assessed by flow cytometry. LT-HSCs are considered to be present in test cells only when recipient PB contains 
Table 1-1. Phenotype of murine hematopoietic stem cell at different development stage.

\begin{tabular}{|c|c|}
\hline HSC & Marker \\
\hline $\begin{array}{l}\text { Hemangioblast (Primitive streak, yolk } \\
\text { sac) }\end{array}$ & Flk-1(Kdr) $^{+}$ \\
\hline Pre-HSC (AGM) & $\mathrm{CD} 34^{+} \mathrm{CD} 41^{+} \mathrm{Sca}-1^{-} \mathrm{CD} 45^{-}$ \\
\hline HSC (AGM, placenta) & $\mathrm{CD} 34^{+} \mathrm{CD} 45^{+} \mathrm{CD} 41^{+/-} \mathrm{Sca}-1^{+/-}$ \\
\hline HSC (fetal liver) & 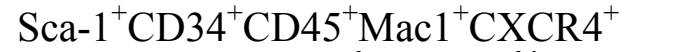 \\
\hline \multirow[t]{8}{*}{ HSC (bone marrow) } & c-kit $^{+}$Thy1.1(CD90) ${ }^{\text {lo }}$ Lin-Sca $^{\text {hi }}$ (KTLS) \\
\hline & Sca- $1^{+} \mathrm{CD} 34^{+/-} \mathrm{CD}_{4} 5^{+} \mathrm{Lin}^{-}$ \\
\hline & $\mathrm{CD} 150^{+} \mathrm{CD} 48^{-} \mathrm{CD} 244^{-}$(SLAM) \\
\hline & Rh123 ${ }^{\text {low }}$, Hoe $33342^{\text {low }}$,Pyronin Y ${ }^{\text {low }}$ \\
\hline & 5-FU resistant \\
\hline & Aldehyde dehydrogenase $^{\text {high }}$ \\
\hline & Fr25 (Small cells) Lin \\
\hline & Side-population cells \\
\hline
\end{tabular}

AGM, aorta gonado mesonephros; 5-FU, fluorouracil; HSC, hematopoietic stem cell; SLAM, signaling lymphocyte activation molecule.

Reprinted with permission. Ratajczak MZ. Phenotypic and functional characterization of hematopoietic stem cells. Curr Opin Hematol. 2008;15(4):293-300. ${ }^{42}$ 
donor- derived cells in the all triple lineages after at least 16 weeks post transplantation. Long-term repopulating assay also can be used to evaluate HSC activity in genome modified cells by retroviral delivery with fluorescence markers or leukemia-initiating cells (LICs) from leukemia samples.

\section{Competitive repopulation assay}

In competitive repopulation assay test cells and standard cells are mixed at variety of ratio, 1:1 ratio at most cases, and injected into hosts. Usually standard cells contain specific number of whole bone marrow cells or HSCs which allows semi-qualify HSC frequency relative to standard cells. Test cells and standard cells should be distinguishable from each other. For more accurate evaluation of competitive reconstitution unit (CRU) frequency limiting-dilution assay is used.

\section{Limiting-dilution assay}

Limiting-dilution assay allows evaluation of absolute CRU frequency (HSC number) in test cells. It involves a titration of varying dose of test cells (at least 3 doses), which are mixed with $1-2 \times 10^{5}$ competitors (contain about 5-10 CRU) and injected into lethally irradiated hosts. Competitors are usually subjected to two rounds of bone marrow transplantation prior to the limiting-dilution assay. The proportion of recipients whose bone marrow cells are determined to contain $>1 \%$ or $>5 \%$ cells of test cell origin in all three lineages (myeloid lineage, T lymphocyte lineage and B lymphocyte lineage) after at least 16 weeks following transplantation is used to calculate the CRU frequency generated by Poisson statistics.

\section{Serial transplantation assay}

Given population is transplanted into primary recipients, whose bone marrow cells were harvested and transplanted into secondary recipients and further into tertiary recipients. Serial transplantation assay is the most stringent HSC assay since only the most primitive HSCs are able to yield long-term, multilineage repopulation through multiple rounds of transplantation.

\section{Regulation of Hematopoietic Stem Cell Pool}

HSCs give rise to all the blood lineages throughout the life span; as a result HSC pool should be well maintained. This usually involves the regulation of the balance of HSC self-renewal and differentiation, HSC survival and death (apoptosis/necropsies), lineage priming and selection and many other aspects of HSC activities by either intrinsic or extrinsic signals or both. Intrinsic mechanism includes transcription factors, epigenetic 
modification, non-coding RNA, especially microRNA and extrinsic signals come from microenvironment (bone marrow niche) where HSCs reside.

\section{Transcription factors}

Numerous of transcription factors are shown to play critical role in HSC selfrenewal or differentiation through gain of function or loss of function assays. They include HOX genes, Gata2, Runx1, Gfil and many others. HOX genes are highly conserved from Drosophila to mammals and play roles in embryonic patterning and hematopoiesis. The detail of $H O X$ gene function in normal and malignant hematopoiesis will be discussed in later paragraphs. Gata2 is one of the six members of GATA family in mammals. It is expressed in primitive hematopoietic cells and its expression level declines gradually along hematopoietic differentiation. ${ }^{43,44}$ Gata2 knock-out mice die at day 10-11 of gestation due to impaired primitive hematopoiesis, demonstrating that Gata2 is essential for earliest stage of hematopoiesis during embryogenesis. ${ }^{43,44}$ Gata2 is also crucial for adult HSCs and HPCs proliferation. Gata 2 may preserve immature cell pool by inhibiting hematopoietic precursor cell differentiation and also confers increased quiescence of human HSCs and HPCs when expressed at high level. ${ }^{45,46}$ At meanwhile, reciprocal expression pattern of Gatal and Gata2 is critical for erythropoiesis and erythroid homeostasis. Gata2 may function through SCL and active both Kit and Gata2 in immature erythroid cells. ${ }^{47}$

\section{Epigenetic regulation}

Epigenetic regulation, histone or DNA modification, allows changes of large set of gene expression by influencing the accessibility of transcription factors to DNA and altering transcription profile of cells. Epigenetic regulation includes methylation, acetylation, phosphorylation, sumoylation and ubiquitylation. Here we focus on the discussion how histone or DNA epigenetic modification regulates or maintains HSC signature.

Histone hyperacetylation usually indicates 'open' chromatin whereas deacetylation means 'condensed' chromatin. Histone acetylation is catalyzed by histone acetyl transferase and histone deacetylation is catalyzed by histone deacetlylases (HDACs). Histone methylation usually occurs on arginine or lysine residues. Methylation on $\mathrm{H} 3 \mathrm{~K} 4, \mathrm{H} 3 \mathrm{~K} 36$ and $\mathrm{H} 379$ are associated with transcription activation while methylation on $\mathrm{H} 3 \mathrm{~K} 9, \mathrm{~K} 3 \mathrm{~K} 27$ and $\mathrm{H} 4 \mathrm{~K} 20$ are related to transcription repression. BMI1 belongs to Polycomb-group (PcG) proteins, which repress gene (such as $H O X$ genes) transcription through histone modification. Bmi- $1^{-/-}$mice demonstrated reduced number of adult HSCs but not fetal liver HSCs and defect in long-term reconstitution of both fetal liver and adult $\mathrm{HSCs}^{48}$ while enforced expression of Bmi-1 augments HSC selfrenewal, ${ }^{49-51}$ indicating Bmi-1 is essential for self-renewing HCSs. Besides HSCs, Bmi-1 is also required for neuron stem cells self-renewal but not survival or differentiation. ${ }^{52} \mathrm{~A}$ similar phenomenon was observed in the Mph1/Rae28 loss mice ${ }^{53}$ and MPH1/RAE28 is 
another member of PcG family which forms ploycomb complex 1 with M33, BMI1 or MEL18 and SCMH1. ${ }^{54-58}$ In contrast, Ring $1 b$ loss mice ${ }^{59}$ or mice with hypomorphic mutation of Eed ${ }^{60}$ or Suz $12^{61,62}$ exhibited enhanced HSC or HPC activities.

Methylation of CpG in DNA is another major epigenetic modification. DNA methylation is catalyzed by DNA methyltransferase (Dnmt1, Dnmt3a and Dnmt3b) and methylation in promoter region is associated with gene silencing. Dnmt3a and Dnmt3b execute de novo DNA methylation and play role in the regulation of cell differentiation. CD34-LSK cells express both Dnmt3a and Dnmt3b. Disruption of Dnmt3a or Dnmt3b or both do not affect either myeloid or lymphoid lineage differentiation potential while loss of both Dnmt $3 a$ and Dnmt $3 b$ but not either one of them result in defect in long-term HSC reconstitution. ${ }^{63}$ Later Challen et al ${ }^{64}$ applied serial transplantation on Dnmt $3 a$-null HSCs which demonstrated 200-fold expansion of phenotypic HSCs in secondary recipients coupled with declined multi-lineage differentiation capacity with successive rounds of transplantation. Expansion of Dnmt3a-null HSCs was associated with activation of HSC self-renewal program including Runx 1 and Gata3 indicating the essential role of Dnmt3a in silencing self-renewal genes in HSCs and permission of hematopoietic

differentiation. ${ }^{64}$ Conditional knockout of Dnmt1 led to defects of HSC self-renewal, bone marrow niche retention and generation of myeloid-restricted progenitors from HSCs. ${ }^{65}$ Haploinsufficency of Dnmtl was shown to be sufficient to impair self-renewal of MALL-AF9-induced leukemia stem cells (LSCs) and similar effect ${ }^{66}$ was seen in BLymphoid leukemia with Dnmt1 hypomorphic allele. ${ }^{67}$ These studies indicated the role of Dnmt1 in normal and malignant HSCs for regulation of self-renewal.

\section{Signaling pathways}

\section{Wnt signaling pathway}

Wnt signaling regulates both HSC self-renewal and differentiation. In mammals Wnt proteins are present in primitive and definitive hematopoiesis sites. Wnt-5a and Wnt-10b are expressed in murine yolk sac (E11), fetal liver (E14) and fetal liver HSCs. ${ }^{68}$ In vitro culture Wnt-5a was shown to have positive effect on proliferation of fetal liver HSCs in an autocrine and paracrine manner, while monocyte and erythrocyte formation were preferred from bone marrow HPCs in the present of soluble Wnt-5a. ${ }^{69}$ Overexpression of $\beta$-catenin, downstream executor of the Wnt signaling cascade, enhances HSC self-renewal. ${ }^{70}$ B-catenin also has been shown to promote T-ALL cells survival, adhesion and proliferation, and it is undetectable in mature $\mathrm{T}$ cells. ${ }^{71}$ Whether $\beta$ catenin shared the same mechanism on HSCs self-renewal and leukemic cells proliferation is not yet clear. 


\section{Notch signaling pathway}

Notch signaling requires physical interaction of Notch ligands and Notch receptors, which are expressed in Notch-ligand-expressing cells and Notch-expressing cells, separately. In bone marrow, HSCs express Notch and bone marrow stromal cells, endothelial cells and fibroblasts, express Notch ligands, such as Jagged and Delta. Notch signaling is critical for embryonic development as Jag1-deficient mice are embryonic lethal due to the defects in the vasculature remodeling. ${ }^{72}$ Notch signaling plays a role in modulating HSCs self-renewal, differentiation and proliferation. Jagged1 alone inhibited proliferation and myeloid colony formation of $\mathrm{CD} 34^{+}$cells but enhanced erythroid colony formation in the presence of stem cell factor (SCF). ${ }^{73}$ Activation of endogenous Notch signaling with immobilized Delta-1 enhanced proliferation of cord blood derived$\mathrm{CD} 34^{+}$cells in vitro and myeloid-lymphoid repopulating ability in vivo. ${ }^{74}$ Expression of the intracellular domain of Notch 1 can immortalized murine HSCs and immortalized cell line was able to differentiate into both myeloid and lymphoid lineages. ${ }^{75}$

\section{Phosphatidylinositol 3 kinase pathway}

Phosphatidylinositol 3 kinase pathway (PI-3) kinase pathway promotes cell growth, proliferation and survival. PI-3 kinase can be activated by receptor tyrosine kinase or other signaling pathway and generate Phosphatidylinositol-3, 4, 5-trisphosphate (PIP3). PIP3 further activates Art. Phosphorated Akt activates mammalian target of rapamycine (mTOR) kinase. mTOR1 promotes cell growth and proliferation, while mTOR2 regulates Akt activity. Pten negatively regulates PI-3 kinase pathway by dephosphorylating PIP3, thus resulting in inhibition of cell growth, proliferation and survival. Conditional knock-out of Pten from embryonic stem cells as well as neuron stem cells can drive cells into cell cycle and enhance self-renewal. ${ }^{76-78}$ Deletion of Pten from adult HSCs also increase cell cycle entry, leading to transient increase of HSC numbers but depletion of HSCs in the mouse bone marrow in the end. ${ }^{79,80}$ Phenotype of Pten deletion/inactivation demonstrated that Pten normally maintains HSCs in a quiescent state. However, Pten deletion also drives leukemogenesis. Yilmaz reported myeloproliferative disease which progressed to AML and ALL when Pten was inactivated in murine hematopoietic system using flox-Mx-1-Cre system. ${ }^{79}$ Different role of Pten deletion on HSCs depletion and leukemic stem cells generation suggests independent mechanism of each phenomena caused by Pten inactivation.

\section{TGF/BMP signaling pathway}

TGF- $\beta$ superfamily includes TGF- $\beta$ /activin/nodal family, bone morphogenetic protein (BMP)/growth and differentiation factor (GDF)/Muellerian inhibiting substance (MIS) family. Upon binding of the ligands, activated type I and type II serine/threonine kinase receptors form heterodimer and phosphorylate specific intracellular receptorregulated Smads (R-Smad), including Smad1,2,3,5, and 8. Smad1, Smad5 and Smad8 are primary mediators of BMP signaling, while Smad2 and Smad3 transduce signal through 
TGF- $\beta$ /activin receptors. Dimer of R-Smads forms complex with common partner, Smad4 , and translocates into nucleus to transcriptionally regulate various target genes in a cellspecific and ligand-dose dependent manner. Inhibitory Smads (I-Smad), Smad6 and Smad7, can negatively regulate TGF- $\beta$ signaling pathway by interfering R-Smads and receptor or R-Smads and Smad4 interaction or recruiting E3-ubiquitin ligases to degrade receptors. Smad1 belongs to BMP signaling pathway. Many studies showed that BMP4 plays a key role in mesoderm induction and hematopoietic commitment and BMP4 knock-out mice displayed defects in mesoderm and blood island formation. ${ }^{81}$ For adult HSCs, BMP4 may act as positive regulator of proliferation and survival. High concentration treatment of BMP4 to human HSCs in vitro can enhance its engraftment in nonobese diabetic-severe immunodeficiency (NOD/SCID) mice. ${ }^{82}$ At meanwhile, BMP4 signaling is also important in sonic hedgehog $(\mathrm{SHH})$-induced human progenitor proliferation. ${ }^{83}$ Different from BMP4, TGF- $\beta 1$ ligand-activated TFG- $\beta$ signaling inhibits HSCs proliferation and induces HSCs apoptosis in vitro. In vivo injection of TGF- $\beta 1$ also inhibits proliferation of murine multipotent hematopoietic progenitors in bone marrow. ${ }^{84}$ As negative regulator of TGF- $\beta$ signaling, Smad 7 is able to block all TGF- $\beta$ signaling pathways. Overexpression of Smad7 increases HSCs repopulating activity in serial transplantation setting. ${ }^{85}$ Increased proliferation induced by Smad7 was not seen in culture. This suggests signaling crosstalk between bone marrow cell and bone marrow niche is required for Smad7 to increase HSCs self-renewal.

\section{MicroRNAs}

MicroRNAs are small, noncoding RNAs which regulate gene expression through transcription or translation repression by interfering initiation of transcription or mRNA destabilization. Dicer is an enzyme which cleaves double-stranded RNA (dsRNA) and pre-microRNA (pre-miRNA) into small interfering RNA or and microRNA respectively. Conditional deletion of Dicer led to loss of HSPCs due to significant increased apoptosis, indirectly suggesting the role of microRNA in HSC maintenance. ${ }^{86}$ Several microRNAs including Mir-125a, Mir-125b, Mir-126, Mir-130 and Mir-155, are highly enriched in $\mathrm{HSCs}^{86-90}$ and dysregulation of some microRNAs are associated with human hematopoietic malignancies such as MDS/AML, ${ }^{91}$ B-cell acute lymphoblastic leukemia ${ }^{92,93}$ and chronic lymphocytic leukemia (CLL). ${ }^{94}$ miR-99b/let7e/miR-125a cluster is highly expressed in LT-HSCs and overexpression of miR-99b-let7e-miR-125a cluster or miR-125a alone was able to enhance HSC long-term multilineage reconstitution. ${ }^{86}$ When overexpressed separately, only miR125a but not miR-99b, let7e was able to amplify HSC pool size in vivo by protecting primitive hematopoietic cells from apoptosis through downregulation of proapoptotic protein Bak1. ${ }^{86} \mathrm{MiR}-125 \mathrm{~b}$ is another microRNA highly expressed in LSK cells. Overexpression of miR-125b demonstrated reconstitution advantage at competitive transplantation setting but caused a dose-dependent myeloproliferative disorder/myeloid leukemia. ${ }^{89}$ The observation of miR125b-induced HSCs expansion was confirmed in Ooi. A,G et al's study, but they further identified that miR-125b preferentially expanded Slamf $1{ }^{\mathrm{lo}} \mathrm{CD} 34^{-} \mathrm{LSK}$ lymphoid-balanced and the Slamf $1^{\text {neg }}$ CD $34^{-}$LSK lymphoid-biased cell, possessing a balanced

myeloid/lymphoid and lymphoid-biased cell output, respectively. ${ }^{88}$ Besides the regulation 
of HSC self-renewal, many microRNAs were implicated in regulating hematopoietic progenitor cells (HPCs) differentiation, including miR-155 for lymphoid and myeloid development, ${ }^{87,95,96}$ mir-233 for myeloid differentiation ${ }^{97,98}$ and the miR-181/miR150/miR-17-92 cluster for lymphoid differentiation. ${ }^{99-102}$ Taken together, microRNAs regulate hematopoiesis at multiple levels including HSC self-renewal, HSC fate decision and HPC differentiation.

\section{HOX Gene Family}

HOX genes are homeodomain-containing transcription factors. They were initially characterized in Drosophila and involved in process of body pattern during the embryo development as well as many adult processes including hematopoiesis. Hox gene products share evolutionally conserved 60-amino-acid homeobox domain, which specifies basic Helix-turn-helix DNA binding domain. Sequences flanking the homeodomain have been shown to have either activation or suppression functions. In Drosophila the HOX genes map to the antennapedia complex (Ant-C) and Bithorax complex (BX-C), which are referred to HOM-C. ${ }^{103-105}$ Antp class genes include labial (lab), proboscipedia ( $\mathrm{pb}$ ), Deformed (Dfd), Sex combs reduced (Scr), Antennapedia (Antp), Ultrabithorax (Ubx) and abdominal-A (abd-A) (Figure 1-3). Abdominal-B (Abd$\mathrm{B})$ is another homeotic selector gene and it is related to Antp class by its sequence but not structure. Lab, the most 3' gene is required for the most anterior HOM-C functional domain while the most 5' gene of the complex, $\mathrm{Abd}-\mathrm{B}$, is required for the posterior abdominal segment development. In human and murine genomes there are 39 class I Hox genes due to genome duplication. They are referred to HOXA, HOXB, HOXC, and HOXD clusters, each of which contains 9 to 11 groups located on human chromosomes 7, 17, 12 and 2 or mouse chromosome 6, 11, 15 and 2 (Figure 1-3). ${ }^{96,97}$ In mammals Hox genes carrying the same number in different clusters are paralogous and the paralogy is complete only in groups 13, 9, and 4, which contain all four genes. All Hox genes have the same 3'-to-5' orientation and according to their position within their respective gene cluster 3' Hox genes are expressed early on and at anterior locations whileas 5' Hox genes are expressed later developmental stage and at posterior locations during the development. The correspondence between gene topology and the place and time of transcription are referred to as spatial and temporal collinearities, respectively.

\section{HOX gene upstream regulators}

Retinoic acid, Trithorax group (Trx-G) and Polycomb group (PcG) genes were shown to be upstream regulators of HOX genes in hematopoietic and non-hematopoietic tissues. Due to lack of the evidence showing that retinoic acid treatment regulates HOX gene expression in hematopoietic cells, here we focus Trx-G and PcG genes.

Trithorax is required for the maintenance of HOM-C genes expression in the Drosophila. ${ }^{106}$ Mixed lineage leukemia (MLL) is the mammalian homolog of Trithorax gene and is usually seen as part of fusion genes in leukemia such as infant biphenotypic 


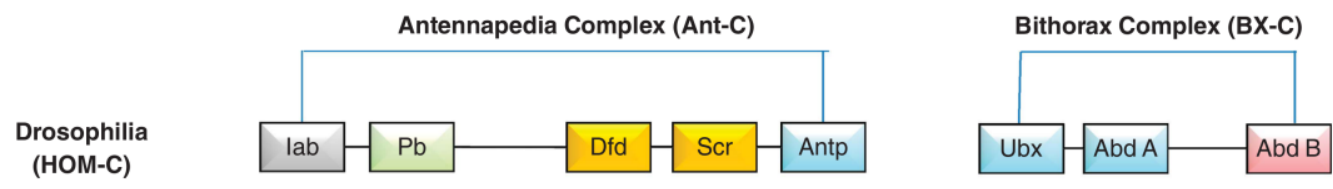

Chromosome number

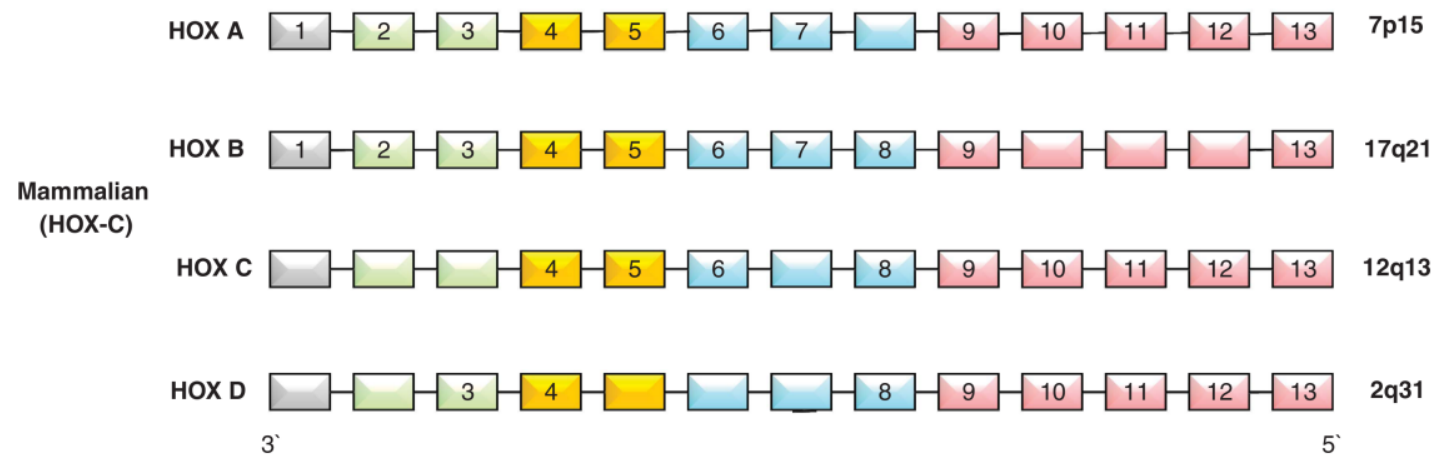

Figure 1-3. A schematic structure of clustered HOX genes.

The 39 HOX genes are located on four different chromosomes. Homology of human HOX genes HOX-C to Drosophila HOM-C genes is shown by colors. Blank squares show missing genes. They play critical role in body patterning during embryo development.

Reprinted with permission. Alharbi RA, Pettengell R, Pandha HS, Morgan R. The role of HOX genes in normal hematopoiesis and acute leukemia. Leukemia. 2013;27(5):1000$1008 .^{107}$ 
leukemia ${ }^{108}$ and etoposide-induced leukemia. ${ }^{109} \mathrm{Mll}$-null mice are embryonic lethal due to morphogenetic defects and hematopoietic abnormality. ${ }^{110}$ Disrupted Hox gene expression pattern along the vertebral axis due to loss of $\mathrm{Mll}$ was also observed. ${ }^{110} \mathrm{Mll}$ null fetal liver HPCs showed reduced colony forming capacity and it can be due to the effect of loss of $\mathrm{Mll}$ or disrupted downstream $\mathrm{HOX}$ genes on the growth of hematopoietic precursors. ${ }^{111}$

As mentioned in previous paragraph $\mathrm{PcG}$ proteins maintain HOX genes in a repressed status through histone modification. $\mathrm{PcG}$ genes are preferentially expressed in differentiated bone marrow cells except Bmi-1, ${ }^{112}$ which is consistent with their role in repressing HOX gene expression. Bmi-1 and Mph1/Rae 28 are two well-studied PcG genes regarding their roles in hematopoiesis and these studies were discussed in previous section.

\section{Hox cofactors}

Homeodomain HOX proteins recognize and bind to specific TAAT DNA consensus site which confers HOX genes limited specificity. HOX proteins cooperatively bind to DNA with PBC, a group of three animo acid loop extension (TALE) homeodomain-containing proteins. PBC gene family includes the pre-B-cell leukemia-1 $(\mathrm{Pbx}-1)$ and myeloid ectopic insertion site (Meis) families. ${ }^{113-116}$. HOX cofactors provide further specificity and affinity of HOX proteins. PBX proteins do not have strong proteinDNA interaction, but they are able to form protein complex with HOX proteins from paralog group 1 to 10, thus providing DNA-binding specificity of HOX proteins from different paralog groups. ${ }^{116,117} \mathrm{HOX}$ proteins from paralogs 1 to 8 form protein complex with PBX through a tryptophan within a conserved YPWM motif ${ }^{18-121}$ while HOX proteins from paralogs 9 to 10 utilize the tryptophan in a conserved ANW domain. ${ }^{121}$ HOX proteins which belong to paralogs 11-13 prefer to form complex with MEIS1 homeodomain proteins other than PBX, ${ }^{121,122}$ which was frequently detected in the leukemia arising in $\mathrm{BXH} 2$ mice. ${ }^{123}$ However, dimeric complex of MEIS/MEIS-like protein and PBX ${ }^{124-129}$ and trimeric complex of MEIS/MEIS-like protein-PBX$\mathrm{HOX} / \mathrm{HOX}$-like protein ${ }^{128-130}$ were also formed in myeloid leukemia cell lines or nonhematopoietic tissues.

\section{Role of Hox genes in normal hematopoiesis}

HOX genes are expressed in HSCs and progenitors in a lineage-specific or differentiation stage-specific manner. Genes of HOXA clusters are more likely expressed in myeloid cells, HOXB genes in erythroid lineage and HOXC genes in lymphoid cells. HOXD genes play roles in limb development during embryogenesis but not in hematopoiesis since lack of expression of HOXD gene in hematopoietic cells. ${ }^{131-133}$ Anterior 3' end HOX genes including HOX1-6 are highly expressed in primitive HSCs and are downregulated along the differentiation while posterior 5 ' end $\mathrm{HOX}$ genes are mostly expressed in committed hematopoietic cells. ${ }^{134}$ 
Evidence has been provided by the loss of function and gain of function studies of Hox genes in mouse models. HOX genes are actively involved in both embryonic and adult hematopoiesis, maintaining or enhancing HSC self-renewal as well as blocking or facilitating myeloid/lymphoid lineage differentiation (Table 1-2). Due to genetic interactions between HOX clusters, results of knockdown or knockout studies sometimes do not reflect the findings of overexpression studies.

Knockdown of human HOXA5 in hematopoietic organs led to increased erythroid progenitor number and reduced myelomonocytic cells, while constitutive expression of HOXA5 increased myeloid progenitor numbers and blocked erythroid differentiation. ${ }^{135,136}$ Hoxa 7 null mice displayed reduced megakaryocyte/erythroid progenitors with reticulocytosis and thrombocytopenis, suggesting a role in megakaryocyte and erythrocyte development. ${ }^{137}$ Knockout Hoxa9 caused more severe phenotype than Hoxa 7. Hoxa9 null mice displayed reduced lymphoid and myeloid cells together with reduction in CLPs, CMPs and GMPs. ${ }^{137}$ Though aberrant expression of Hoxa 7 and Hoxa9 is the feature of leukemia harboring MLL mutations, Hoxa 7 and Hoxa 9 are not necessary for MLL-GSA7-mediated leukemogenesis. ${ }^{137}$ Overexpression of Hoxa9 increased HSC and myeloid progenitor pools and reduced pro-B progenitors. ${ }^{138}$ and further led to myeloid leukemia with long latency through collaboration with MEIS1a but not PBX1b. ${ }^{139}$ Similar to Hoxa9, Hoxa10 was identified as pro-oncogenic gene which can lead to increased myeloid blast cells in vitro, blocked erythroid differentiation in methylcellulose, reduced B cell differentiation and enhanced myelopoeisis in vivo when overexpressed in human purified $\mathrm{CD} 34^{+}$hematopoietic progenitor cells. ${ }^{140}$ HOXB4 was shown to be one of the most promising factors which can expand murine HSC in vitro and in vivo without compromising HSC multilineage differentiation potential at a dose-dependent manner. ${ }^{141}$ However Hoxb4 knock-out studies showed lacking entire Hoxb4 gene had little effect on hematopoiesis, with mild reduction in the number of HPCs and HSCs in adult bone marrow and fetal liver without multilineage differentiation perturbation. ${ }^{32}$ Other Hoxb genes were shown to be expressed at 2.3-3.6 fold higher level in c-Kit ${ }^{+}$fetal liver cells of Hoxb4 null mice, while the expression level of Hoxb4 paralogs were not altered (Hoxa4, Hoxd4) or even reduced (Hoxc4) ${ }_{142}^{142}$ suggesting other Hoxb genes' but not Hoxb4's paralogs' duplicate roles in hematopoiesis in Hoxb4 null mice. Biji ${ }^{142}$ et al also showed that loss of the Hoxb entire cluster had little effect on murine fetal liver HSC self-renewal and differentiation potential. c-Kit ${ }^{+}$fetal liver cells from Hoxbl ${ }^{-} \mathrm{Hoxb}^{-/}{ }^{-}$demonstrated downregluation of almost all Hoxa genes expect Hoxa 13, upregulation of Hoxc4, Hoxc9 and Hoxc1 1, ${ }^{142}$ indicative a complex network of genetic interaction between Hox genes in hematopoietic cells. Roles of Hoxb3, Hoxb6, Hoxc3, HOXC4 and Hoxc8 in HSC maintenance and myeloid, lymphoid and erythroid differentiation were elaborately described in many other studies. ${ }^{143-149}$

\section{Role of Hox genes in acute leukemia}

Though Hox genes were showed to play roles in normal hematopoiesis, numerous studies have shown that aberrant expression of some $H O X$ genes are highly associated 
Table 1-2. HOX gene studies.

\begin{tabular}{|c|c|c|c|c|}
\hline HOX gene & Gain of function & Loss of function & Species & Reference \\
\hline HOXA5 & $\begin{array}{l}\uparrow \text { Myeloid progenitors and block erythroid } \\
\text { differentiation. }\end{array}$ & $\begin{array}{l}\uparrow \text { Erythroid progenitors and } \\
\downarrow \text { myelomonocytic cells. }\end{array}$ & Human & 135,136 \\
\hline Hoxa7 & & $\begin{array}{l}\downarrow \mathrm{MEP} \text {, reticulocytosis and } \\
\text { thrombocytopenia. }\end{array}$ & Mouse & 137 \\
\hline Hoxa9 & $\begin{array}{l}\uparrow \text { HSCs expansion and myeloid progenitor } \\
\text { proliferation. Block erythroid } \\
\text { differentiation and } \downarrow \text { pre-B-cell } \\
\text { differentiation. }\end{array}$ & $\begin{array}{l}\downarrow \downarrow C M P, \text { GMP, CLP, lymphoid } \\
\text { precursors, repopulating ability } \\
\text { and } \downarrow \text { spleen cellularity and size. }\end{array}$ & Mouse & $137-139,150$ \\
\hline HOXA10 & $\begin{array}{l}\uparrow \uparrow \text { Blasts cells and myelopoiesis, } \downarrow \text { B-cell } \\
\text { differentiation and block erythroid } \\
\text { differentiation }\end{array}$ & & Human & 140 \\
\hline Hoxb3 & $\begin{array}{l}\text { Block B and T-cell differentiation and a } \\
\text { delay in myeloid precursor proliferation. }\end{array}$ & $\begin{array}{l}\downarrow \downarrow \text { B-cell progenitors and BM } \\
\text { cellularity. }\end{array}$ & Mouse & 143,144 \\
\hline HOXB4/hoxb4 & $\uparrow \uparrow H S C s$ expansion. & $\begin{array}{l}\downarrow \downarrow \text { Hematopoietic organs } \\
\text { cellularity and size, } \downarrow \text { HSCs and } \\
\text { HPs and } \uparrow \text { Hoxb genes. }\end{array}$ & $\begin{array}{l}\text { Human/ } \\
\text { Mouse }\end{array}$ & $141,142,151,152$ \\
\hline Hoxb3/b4 & & $\downarrow \downarrow$ HSCs and HPs & Mouse & 153 \\
\hline Hoxb6 & $\begin{array}{l}\uparrow \text { HSCs expansion and myeloid precursor } \\
\text { proliferation. } \downarrow \text { Erythropoiesis and } \\
\text { lymphopoiesis }\end{array}$ & $\uparrow$ Early erythroid progenitors. & Mouse & 146,147 \\
\hline Hoxc3 & & $\downarrow$ Erythroid progenitors. & Mouse & 149 \\
\hline $\mathrm{HOXC4}$ & $\begin{array}{l}\uparrow \text { Early and committed myeloid and } \\
\text { erythroid progenitors. }\end{array}$ & & Human & 148 \\
\hline Hoxc8 & & $\begin{array}{l}\downarrow \downarrow \text { Erythroid, granulocytes and } \\
\text { marcrophage colony formation } \\
\text { potential. }\end{array}$ & Mouse & 145 \\
\hline
\end{tabular}




\section{Table 1-2. (Continued).}

Abbreviations: CLP, common lymphoid precursors; CMP, common myeloid progenitors; GMP, granulocyte/monocyte precursors; HOX, homeobox; HP, Hematopoietic progenitors; HSCs, Hematopoietic stem cells; MEP, megakaryocytic/erythroid progenitors.

Reprinted with permission. Alharbi RA, Pettengell R, Pandha HS, Morgan R. The role of HOX genes in normal hematopoiesis and acute leukemia. Leukemia. 2013;27(5):1000-1008. ${ }^{107}$ 
with hematopoietic malignancies through forming HOX fusion proteins, dysregulation by their upstream regulators or other factors.

Since NUP89-HOXA9 fusion protein was identified in $\mathrm{t}(7 ; 11)(\mathrm{p} 15 ; \mathrm{p} 15)$ positive human myeloid leukemia ${ }^{154,155}$ due to chromosome translocation, many other HOX genes have been shown to be NUP98 translocation partners including HOXA11, ${ }^{156,157}$ HOXA13, ${ }^{156} \mathrm{HOXD} 11,{ }^{158} \mathrm{HOXD} 13^{159}$ and $\mathrm{HOXC11}{ }^{160}$ in human acute myelogenous leukemia (AML) and T-cell acute lymphocytic leukemia (T-ALL). NUP98 is a nuclear pore complex protein and in NUP98 fusions N-terminal GLFG repeats of NUP98 gene joins the C-terminal portion of $H O X$ genes including homeodomain. NUP98 fragment may lead HOX protein to bind to inappropriate target genes or dysregulate HOX target gene expression. Retroviral delivery of $N U P 98-H O X$ fusion genes were proved to cause myeloproliferative disease or AML in transplanted mice. ${ }^{161-163}$ Co-expression of MEIS1 can shorten the latency of NUP98-HOXA9-mediated AML in transplanted mice from 230 days to 142 days $^{163}$ and induce myeloblastic leukemia together with NUP98$H O X D 13$ fusion gene with latency of 75 days which was not seen in mice transplant with cells only transduced with NUP98-HOXD13 alone. ${ }^{162}$ Combination of NUP98-HOXA9 and BCR-ABL or TEL-PDGF $\beta R$ induced AML in transplanted mice which can't be induced by NUP98-HOXA9 alone or BCR-ABL alone or TEL-PDGF $\beta$ R alone. ${ }^{164}$

Aberrant expression of $H O X$ gene without chromosome translocation was also actively involved in hematopoietic malignancies including AML and acute lymphoid leukemia (ALL). It is usually caused by $H O X$ gene upstream regulator. MLL maintains $H O X$ gene expression by direct binding to the proximal promoter. ${ }^{165}$ Oncogenic MLL fusion proteins activate their transcriptional target at higher level than wild-type MLL ${ }^{166}$ and usually upregulate 5' end Hoxa genes other than 3' end Hoxa genes in mouse immortalized cell lines in combination of Meis $1 .{ }^{167}$ Earlier studies indicated expression of Hoxa genes and Meis 1 was required for MLL fusion proteins transforming activity, ${ }^{167}$ but there are evidence showing involvement of Hoxa genes and Mesil varies in different MLL fusion protein-induced leukemias. MLL-ENL and MLL-CBP were not able to transform hematopoietic cells in Hoxa 9 and Hoxa 7 null background ${ }^{167,168}$ while Hoxa 7 and Hoxa9 do not contribute to the initiation of MLL-GAS7- and MLL-AF9-mediated leukemogenesis in mice. ${ }^{137,169}$ The possibility is that non-Hox gene targets of MLL fusion protein are sufficient to induce leukemias in mice, thus Hoxa9 or Hoxa 7 are not long required for these MLL fusion protein-mediated leukemogenesis. $C D X 2$ gene is another $H O X$ gene upstream regulator which is highly expressed in $90 \%$ of human AML cases in the form of either wild-type $C D X 2$ or $E T V$-fused $C D X 2$. Overexpression of $C d x 2$ alone is sufficient to upregulate Hox genes, Hoxb3, Hoxb6, and Hoxb8 or Hoxb9, Hoxa 10, Hoxb5, and Hoxa 7, in mouse myeloid progenitors. ${ }^{170} \mathrm{CDX} 2$ transforming actively is dependent on its N-terminal transactivation domain, which critical for CDX2 to dysregulate Hox gene expression. ${ }^{170}$ Nucleophosmin 1 (NPM1) is the protein located in nucleolus and can be translocated into nucleoplasm during serum starvation or anticancer drug treatment. Normal NPM1 dosen't regulate HOX gene expression. Relocation of NPM1 into cytoplasm $\left(\mathrm{NMPc}^{+}\right)$upregulates a set of $\mathrm{HOX}$ genes in AML cases. ${ }^{171,172}$ But the molecular association of $\mathrm{NPMc}^{+}$and dysregulation of $\mathrm{HOX}$ genes is not yet clear. 


\section{HOXB4}

HOX family members play an important role in body patterning during embryogenesis and demonstrated lineage-specific function through life in variety of tissues including the hematopoietic organs. ${ }^{173}$ In hematopoietic organs Hoxb4 is expressed in mouse $\mathrm{Lin}^{-} \mathrm{Scal}^{+} \mathrm{c}-\mathrm{kit}^{+}$(LSK) cells with long-term repopulating capacity and AGM-derive $\mathrm{CD} 45^{+} \mathrm{CD} 144^{+}$cells which are enriched with HSCs. ${ }^{174}$ With undetectable Hoxb4 expression level in fetal liver HSCs Hoxb4 may not be critical for expansion of fetal liver HSCs. ${ }^{174}$ Hoxb4 expression undergoes down-regulation with hematopoietic differentiation. Specific expression of HOXB4 in HSCs suggests HOXB4's important role in HSC self-renewal. However, loss of Hoxb4 studies revealed mild phenotype which seems to contradict to the observation from HOXB4 overexpression studies. Hoxb4-deficient mice had mild reduction in HSC numbers in both bone marrow and fetal liver without altering cell cycle distribution and compromised competitive repopulating capacity observed in both primary and secondary recipients. ${ }^{152}$ Moderate phenotype seen in Hoxb4-deficient mice may be due to compensation effect from other Hox genes. Or it suggests Hoxb4 plays less prominent role in non-stressed status of hematopoiesis and enhances proliferative rate of HSCs in the transplantation setting which demands higher level of proliferation.

\section{HOXB4 role in embryonic stem cell derived hematopoiesis}

Hoxb4 is selectively expressed in murine AGM-derived HSCs but not fetal liver HSCs, indicating Hoxb4's role in HSCs specification but not amplification during embryogenesis. Cheryl D. Helgason et al $^{175}$ showed overexpression of HOXB4 enhanced erythropoieitic and primitive hematopoietic differentiation potential of mouse ESCs with increased number of erythroid colony-forming (CFC-E) progenitors and mixed-lineage (granulocyte/erythroid/macrophage/megakaryocyte) colony-forming (CFC-GEMM) progenitors in culture. Later on George Daley's group demonstrated that enforced expression of HOXB4 promoted murine embryonic body (EB)-derived CFU-GEMM formation which expressed HSC markers (c-Kit and CD31) and differentiation markers of myeloid, lymphoid and erythroid lineages and conferred cells isolated precirculation murine yolk sac as well as EB-derived hematopoietic cells with myeloid and lymphoid engraftment potential in lethally irradiated mice. ${ }^{176}$ Similar results were also observed by other groups. ${ }^{177}$ When it comes to human ESCs, HOXB4 overexpression by vector transfection and direct delivery of recombinant HOXB4 fusion protein also exhibited the similar outcome on human ESCs, leading to expansion of erythroid and myeloid/monocytic progenitors in culture. ${ }^{178,179,180}$

\section{HOXB4 overexpression enhances adult HSC self-renewal}

Keith Humphries's group showed that overexpression of HOXB4 in murine primitive bone marrow cells by retroviral gene delivery significantly increased CRU frequency in lethally irradiated mice, achieving 1.4 fold increase above normal bone 
marrow values while control cells only led to $3 \%$ recovery of normal value. ${ }^{141}$ Further studies showed HOXB4-transduced HSCs did not expand above normal HSC levels of unmanipulated mice in extended observation, ${ }^{181,182}$ indicating there were counterregulatory signals to regulate HOXB4-mediated HSC expansion in transplanted mice. Besides in vivo expansion of HSCs in transplanted mice, HOXB4 overexpressing HSCs were able to achieve 40-fold net HSC increase in vitro and retain lymphoid-myeloid repopulating potential. ${ }^{183}$ Both retroviral transfer of $H O X B 4$ cDNA and direct delivery of the HOXB4 homeoprotein demonstrated certain level of expansion of human LTC-ICs and NOD-SCID mouse repopulating cells from human cord blood CD34 ${ }^{+}$cells. ${ }^{151,184}$ Later on Xiao-Bing et al ${ }^{185}$ claimed species-specific differences in the potency of HOXB4-mediated HSC expansion which was highly dependent on HOXB4 expression level. HOXB4 expanded canine and mouse CD $34^{+}$cells best and had the least efficiency on human or nonhuman primate $\mathrm{CD} 34^{+}$cells in vitro. ${ }^{185}$ Immortalized mouse hematopoietic cells by HOXB4 maintained short-term and long-term repopulating capacity without developing leukemia. ${ }^{185}$ HOXB4 protein is subjected to CUL4-mediated degradation and degradation-resistant HOXB4 markedly enhanced human $\mathrm{CD}_{3}{ }^{+}$cells maintenance in vitro and in transplanted NOD/SCID/IL2R- $\gamma^{\text {null }}$ (NSG) mice with multilineage engraftment. ${ }^{186}$

\section{HOXB4 role in leukemogenesis}

It is believed that overexpression of HOXB4 enhanced murine HSC expansion in vitro and in vivo without causing hematopoietic disorders or malignancies. ${ }^{141,181-183}$ Compared with other oncogenic HOX proteins, ${ }^{139,143,147,162-164}$ HOXB4 was considered as the most optimal factor to expand HSCs till recent report which described two cases of myeloid leukemias in HOXB4 transplanted large animals. ${ }^{187}$ Primitive status of the leukemia cells was dependent on the sustained HOXB4 expression, indicating HOXB4 overexpression played a key role in the leukemic transformation in these large animals. ${ }^{187}$ Integration site determined by LAM-PCR and activation of integration site nearby genes, PRDM16 and MYB, raised the possibility that gamma retrovirus integration-induced mutagenesis was involved in the leukemogenesis in the three large animals. ${ }^{187}$ So far this is the only report of HOXB4-associated AML and it may due to high level of the HOXB4 expression achieved in the transplanted CD $34^{+}$cells.

\section{Prdm Gene Family}

PRDM (PRDI-BF1 and RIZ homology domain containing) family is a subfamily of the SET domain containing proteins and consists of 16 orthologs in rodents. ${ }^{188}$ They are all characterized by a N-terminal PR domain which is $20-30 \%$ identical to the SET domain present in histone lysine methyltransferase (HMTs) (Figure 1-4). ${ }^{189} \mathrm{PR}$ domain is present in all family members and is followed by repeated zinc fingers except PRDM1 1 (Figure 1-5). ${ }^{189}$ Although most PRDM proteins contain PR domains, HMT activity was only detected in the PR domains of PRDM2, PRDM8 and PRDM9. ${ }^{190-192}$ PRDM2 and PRDM8 were reported as repressive di-methylation of lysine9 of histone 


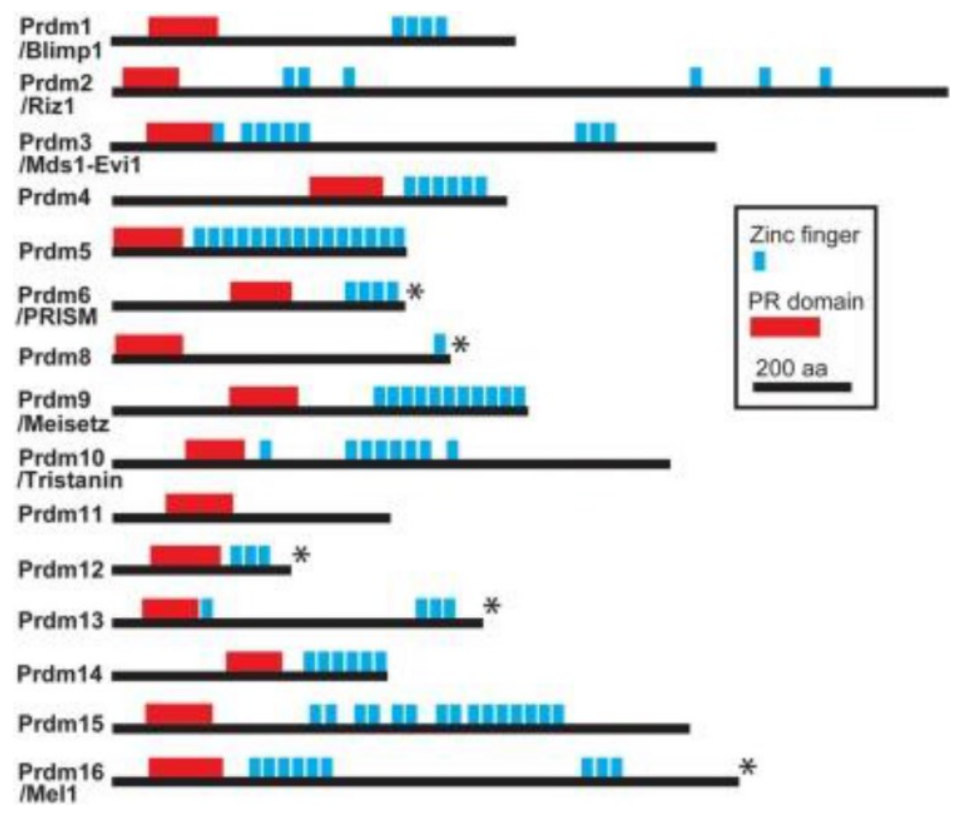

Figure 1-4. The domain structure of the mouse PRDM1-16 proteins.

Illustrations of the protein domain structure of the PRDM family members 1-16. These illustrations are drawn to scale based on a sequence analysis. Zinc fingers are represented by blue boxes and the PR domain by a red box. Those proteins for which a nervous system specific expression pattern is reported in this study are indicated with asterisks.

Reprinted with permission. Kinameri E, Inoue T, Aruga J, et al. Prdm proto-oncogene transcription factor family expression and interaction with the Notch-Hes pathway in mouse neurogenesis. PLoS One. 2008;3(12):e3859. ${ }^{189}$ 


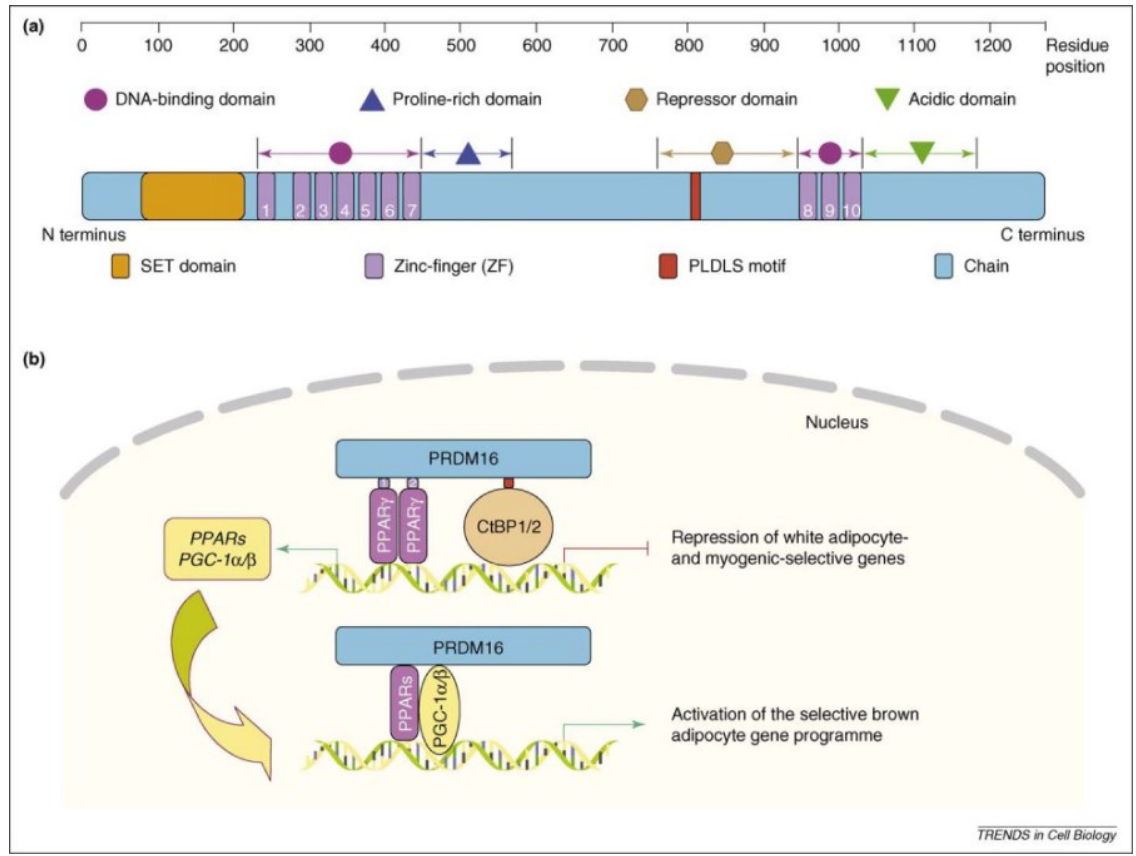

Figure 1-5. Structural and regulatory features of PRDM16.

(A) PRDM16 domain structure and binding sites. After the N-terminal PR domain, the protein has a zinc finger DNA binding domain, a proline-rich domain, a repressor domain, another zinc finger DNA-binding domain, and a $\mathrm{C}$-terminal acidic domain. The PR region corresponds to a SET domain, an 130-amino acid, evolutionarily conserved sequence motif with histone methyltransferase activity. The ten zinc finger domains correspond to the classical $\mathrm{C} 2 \mathrm{H} 2$-type, in which the first pair of zinc-coordinating residues are cysteines, and the second pair are histidines, conferring zinc-dependent DNA- or RNA-binding properties. Two regions in PRDM16, namely zinc-finger (ZF)1 and ZF2, have been identified to bind to PPARg, whereas C-terminal-binding proteins (CtBPs) have been shown to bind to a PLDLS motif at. (B) Schematic representation of brown adipocyte determination by PRDM16

Reprinted with permission. Fruhbeck G, Sesma P, Burrell MA. PRDM16: the interconvertible adipo-myocyte switch. Trends Cell Biol. 2009;19(4):141-146. ${ }^{193}$ 
H3 (H3K9me2) HMTases ${ }^{191,194}$ while PRDM9 as activating tri-methylation of lysine4 of histone H3 (H3K4me3) HMTases. ${ }^{192}$ Other PRDM proteins without intrinsic methyltransferase activity recruit histone-modifying enzymes to achieve epigenetic regulation of gene expression, including HMTs, PRMT5 (Protein methyltransferase 5), LSD1 (lysine-specific demethylase1, Kdm1a), histone deacetylase (HDACs) and histone acetyltransferase (HATs) p300/CBP and P/CAF, by forming protein complex or through their own zinc figure domains or proline-rich domains. Besides histone-modifying enzymes, PRDM proteins also recruit histone-modifying enzyme-associated corepressors. CtBP (C-terminal binding protein) is one of the co-repressors frequently bound by PRDM2, PRDM3 and PRDM16 though their PLDLS CtBP-binding sites. ${ }^{195-201}$ PRDM protein also can bind to DNA directly through their zinc finger domains. Zinc fingers of PRDM1 are able to recognize specific DNA binding sequence GAAAG and mediate PRDM1-DNA interaction. ${ }^{202}$ PRDM3 contains two zinc finger domains: the Nterminal zinc fingers and $\mathrm{C}$-terminal zinc fingers (Figure 1-4). N-terminal zinc fingers prefer to bind a GATA-like motif further from transcription start site (TSS) ${ }^{203}$ and Cterminal zinc fingers bind an ETS-like sequence which is close to TSS. ${ }^{204}$ These two binding patterns of PRDM3 are exclusive to each other which means PRDM3 only binds to either of these two motifs, but not to both at the same promoter. In summary PRDM proteins regulate gene expression as HMTs or through recruiting other HMTs or direct bind to gene expression regulatory elements. Forming complex with a wide range of different HMTs or selective target binding sites provide Prdm proteins a contextdepended regulation activity.

PRDM proteins play roles in normal tissue specification as well as cancer and other diseases. PRDM1 is primarily identified as transcription repressor and it maintains photoreceptor identity and neonatal enterocyte by repressing alternative differentiation programs. ${ }^{205-209}$ Even more PRDM1directs and maintains mast cell differentiation through maintaining other differentiation initiation at a repressive state. ${ }^{210}$ During plasma cell differentiation PRDM1 directly represses Myc through recruitment of HDACs ${ }^{211}$ and represses Ciita through LSD1. ${ }^{212}$ PAX5 and BCL6, both are required for B cell fate specification, are downregulated by PRDM1 during plasma cell differentiation. RNAi targeting $\operatorname{Prdm}_{16^{213,214}}$ and Prdm 16 mutant studies ${ }^{214}$ demonstrated that Prdm16 is essential for brown adipose tissue (BAT) maturation by preventing skeletal myocytes respecification and white adipose (WAT) tissue differentiation. Enforced expression of Prdm 16 induced brown adipogenesis from myoblasts by activating PPAR $\gamma$ transcriptional function through direct protein-protein interaction. ${ }^{214}$ Many PRDM proteins also play roles in primordial germ cells and hematopoietic stem cells. The fact that certain cancer cells share the self-renewal machinery with normal stem cells indicates dysregulation of PRDM may be involved in tumorigenesis. Prdm3 and Prdm 16 maintain long-term and short-term hematopoietic cells. ${ }^{215-218}$ However, both PRDM3 and $P R D M 16$ were original identified in acute myeloid leukemias. Expression of PRDM3 and $P R D M 16$ that lack partial PR domain leads to myeloid leukemia and adult T-cell leukemia due to chromosome translocation events or viral vector insertional activation. ${ }^{219-227} \operatorname{Prdm} 1$ and $\operatorname{Prdm} 14$ are essential for pluripotent primordial germ cells (PGCs) generation and Prdm 1 mutants and Prdm 14 mutants were not able to generate PGCs. ${ }^{228-231} \operatorname{Prdm} 14$ is also required to maintain mouse ESCs at pluripotent state even 
though $\operatorname{Prdm} 14$ is not required for mouse ESC derivation. Knockdown of Prdm 14 in mouse ESCs led to downregulation of ESC genes and activation of extraembryonic endoderm program. ${ }^{232-234}$ Aberrant expression of PRDM14 was detected in breast cancer due to gene amplification on chromosome $8 \mathrm{q}_{13} 3^{235}$ and human lymphoid neoplasms. Gain of function assay of $\mathrm{Prdm} 14$ in transplanted mice provided the evidence of the association of Prdm 14 with lymphoid leukemia initiation in vivo. ${ }^{236}$

\section{PRDM16 protein domain}

PRDM16, which is also named as MEL1 (MDS1/EVI1 like gene 1), was first characterized from $\mathrm{t}(1 ; 3)(\mathrm{p} 36 ; \mathrm{q} 21)$-positive MDS/AML patient leukemia cells. ${ }^{237}$ MEL1 is highly homologous to MDS1/EVI1 with first 222 amino acid sequence homologous to the PR domain of MDS1 and remaining sequence homologous to the EVI1 (ecotropic viral integration site 1). ${ }^{237}$ There is a PR domain at the N-terminal of MEL1 protein, repressor domain (RD) in the middle of the protein and two DNA binding domains which contain either 7 or $3-\mathrm{C} 2 \mathrm{H} 2$ type zinc figure repeats. PR domain of MEL1 protein is $52 \%$ identical to the N-terminal PR domain of the MDS1 protein and the RD contains a consensus binding sequence for the CtBP $2 .{ }^{237}$ Later study found out there were actually three transcription start sites in $M E L$ gene, one in exon 1 and two in exon2, which generated two protein products, 170kD MEL1 and 150kD MEL1S. ${ }^{238}$ MEL1S is the main isoform expressed in $\mathrm{t}(1 ; 3)(\mathrm{p} 36 ; \mathrm{q} 21)$-positive AML cells and is translated from the internal initiation codon ATG579 in exon $4 .{ }^{238}$ MEL1S protein lacks most of the PR domain compared with MEL1 protein. ${ }^{238}$ Aberrant expression of MEL1S gene due to chromosome translocation and vector insertional activation was associated with MDS/AML and T-cell leukemia. ${ }^{223-226,238-240} \operatorname{Prdm} 16$ is mouse homolog of human PRDM16/MEL1 and its protein structure is shown in Figure 1-5.

\section{PRDM16 expression pattern and functions}

In mouse embryo Prdm 16 mRNA was first detected on E9.5 predominately in the neuroepithelum lining the telencephalic vesicle (TV), mesenchyme of the first branchial arch and dorsal aspect of somites and further expanded to the neuroepithelium of the hindbrain and in the optic stalk (OS), mesenchyme of the second and third branchial arches, limb bud (LB) and the facio-acoustic preganlion complex (FACP) on E10.5. ${ }^{241}$ During E11.5 to E12.5 Prdm16 expression expanded to liver, heart, lung epithelium stomach, duodenum and brain, especially in neuroepithelium lining the third ventricle, Meckel's cartilage (MC) and future nasal spetum (NS) in the orofacial region and dorsal root ganglia (DRG). ${ }^{241}$ Prdm 16 showed much broader expression pattern on E14.5, encompassing mutli tissues including brain, olfactory epithelium (OE), testis, pancreas, kidney adrenal gland (AG), lung, DRG, cartilage primordial of the ribs (CPR), limbs, retina and in the lens also. ${ }^{241}$ Widespread expression of Prdm16 in mouse embryo suggests role of Prdm 16 in tissue/organ specification or growth. 
Denis, R.W et al identified PRDM16 as a SMAD3 binding protein mouse in orofacial tissue during E12.5 to E14.5, but PRDM16/SMAD3 interaction did not significantly alter TGF $\beta$ signaling. ${ }^{242}$ Although the molecular mechanism of Prdm 16 during orofacial development is not clear, great progress has been made to understand the role of Prdm 16 in BAT differentiation. There is evidence showing that PRDM16 functions as a co-regulatory protein controlling molecular switch between white fat and brown fat identity (Figure 1-5). ${ }^{185,201,202} \operatorname{Prdm} 16$ transcript is highly expressed in primary brown fat tissue and immortalized brown fat cell lines and overexpression of PRDM16 was able to induce BAT phenotype from PPAR- $\gamma$ expressing white fat precursors or fibroblasts. ${ }^{213}$ PRDM16-mediated identity switching between white and brown adipose tissue was achieved by binding partner switching. Mouse PRDM16 directly binds to CtBP-1 and CtBP-2 as co-repressors to transcriptionally repress WAT differentiation program through direct interaction with the promoter of white fat specific genes. ${ }^{214}$ When CtBP-1 and CtBP-2 are replaced by PGC- $1 \alpha$ (PPAR- $\gamma$-coactivator- $1 \alpha$ ) and PGC- $1 \beta$ (PPAR- $\gamma$-coactivator-1 $\beta$ ), transcription activity of PGC- $1 \alpha$ and PGC-1 $\beta$ are increased and activate brown fat genes. ${ }^{214}$ However, switching cell identity between skeletal muscle and BAT mediated by PRDM16 in $M y f 5^{+}$myoblasts is not involved in white adipogenesis, suggesting independent molecular mechanism of repression of skeletal muscle differentiation. ${ }^{214}$ Enforced expression of PRDM16/C-EBP- $\beta$ complex in $m y f 5^{+}$ myoblasts is sufficient to initiate BAT differentiation program by inducing the PPAR $\gamma$ and PGC-1 $\alpha$ expression. ${ }^{243}$ Recent study showed euchromatic histone-lysinemethyltransferase 1(EHMT1), the enzymatic component of PRDM16 transcriptional complex, determines BAT fate versus muscle cell lineage by stabilizing PRDM16 protein and controlling $\mathrm{H} 3 \mathrm{~K} 9$ methylation status of the muscle-specific gene promoters. ${ }^{244}$

Besides PRDM16's role in promoting BAT identity, there is also evidence showing that PRDM16 executes important function in developing telencephalon through repressive regulation of Hes $1^{189}$ and functions as $\mathrm{H} 3 \mathrm{~K} 9 \mathrm{me} 1$ methyltransferase to maintain the integrity of mammalian heterochromatin. ${ }^{245}$ In the next two paragraphs I will focus on the Prdm16's role in normal and leukemic hematopoiesis.

\section{PRDM16's role in normal hematopoiesis}

Prdm 16 transcript is preferentially expressed in purified LT-HSC, ST-HSCs and MPPs. ${ }^{215,216}$ Enforced expression of Prdm 16 on mouse adult CD $150^{+} \mathrm{CD}^{2} 8^{-} \mathrm{Lin}^{-}$cells led to enhanced HSC reconstitution capacity and poorly differentiated myeloid cells in recipients' bone marrow, which developed splenomegaly and myeloproliferation 20 weeks post transplantation. ${ }^{246}$ Loss of Prdm 16 depleted fetal liver and new born liver HSPCs, leaving remaining neonatal and adult HSCs with compromised long-term reconstitution capacity and $\mathrm{Prdm} 16^{-/}$HSCs contain increased proportion of HSCs undergoing apoptosis/cell death and active cell division. ${ }^{215,216}$ Gene expression data from Prdm 16 ${ }^{-/}$HSCs suggested that $\operatorname{Prdm} 16$ regulates multiple pathways/regulators to maintain HSC function, including $p 53$ related apoptotic pathway, cell cycle regulator $C d k n l a$, transcription factors critical for HSC maintenance and Wnt signaling pathway components Wnt3a and Ryk206. Gathering all the data, it suggests Prdm16 is required 
for fetal and adult HSC maintenance and overexpression of Prdm16 is able to enhance HSC activity in vivo. However, the fact that enforced expression of Prdm16 in HSCs led to undifferentiated HSCs in vitro and myeloproliferation in recipients unveiled Prdm16's oncogenic potential, which may be related to the function of human PRDM16 in AML. The role of PRDM16/sPRDM16 in hematopoietic malignancies will be discussed in the following paragraphs.

\section{PRDM16's role in leukemic hematopoiesis}

Most evidences of association between PRDM16 and hematopoietic malignancies come from the studies of human AML involved chromosome translocation. Since PRDM16/MEL1 was first isolated from $\mathrm{t}(1 ; 3)(\mathrm{p} 36 ; \mathrm{q} 21)$-positive MDS/AML patient leukemia cells, ${ }^{237}$ there are growing evidences showing $s P R D M 16 / M E L 1 S$, lacking most of PR domain, is the major isoform highly expressed in $\mathrm{t}(1 ; 3)(\mathrm{p} 36 ; \mathrm{q} 21)$-positive MDS/AML as fusion partner with $R P N 1^{238,239}$ and $\mathrm{t}(1 ; 21)(\mathrm{p} 36 ; \mathrm{q} 22)$-positive therapyrelated MDS/AML as fusion partner of RUNX1. ${ }^{224,225,247}$ The RUNX1-PRDM16 gene is highly homologous to MDS1/EVII gene, ${ }^{237}$ suggesting RUNX1-PRDM16 may share similar mechanism in leukemic progress. sPRDM16 is also overexpressed in adult T cell leukemia with normal karyotype leukemias due to hypomethylation of regions surrounding $S P R D M 16$ transcription start site ${ }^{223}$. Instead, the full-length PRDM16 is silenced by DNA hypermethylation of its promoter. ${ }^{223}$ This provided alternative epigenetic mechanism of aberrant and predominant expression of $S P R D M 16$ in T-cell leukemia. Overexpression of $S P R D M 16$ but not full length of $P R D M 16$ led to myeloid leukemia in $p 53$ null mice, indicating the cooperative effects of $S P R D M 16$ and loss of p53 in murine AML pathogenesis. ${ }^{248} \mathrm{Du}$. Y et al showed Prdm16 is one of the frequent MSCV integration spots resulting in aberrant expression of sPRDM16, but not PRDM16, which led to immortalization of murine bone marrow progenitors. ${ }^{227}$ Upregulation of sPrdm 16 due to insertional activation by $\gamma$-retroviral SIN vector is sufficient to induce leukemia in mice. ${ }^{240}$ In summary, sPRDM16 plays role in leukemogenesis through chromosome translocation, promoter hypomethylation or vector insertional mutagenesis although the molecular mechanism is still unclear.

\section{Thesis}

Unlike oncogenic HOX genes like HOXA9 and HOXA10, HOXB4 overexpression can mediate murine HSC expansion in vivo without leading to hematopoietic malignancies. Though many studies were carried out to identify HOXB4 target genes and these studies offered some insights regarding the mechanism of HOXB4-mediated HSPC expansion in vitro, survival and specification of ES cells, there are still questions listed below remaining unknown regarding HOXB4-mediated HSC expansion. 


\section{Questions}

1. What are HOXB4's transcriptional target genes in transplanted mice?

2. What are the pathways HOXB4 functions through to achieve robust HSC expansion in vivo?

3. What is the mechanism by which HOXB4 avoids leukemogenesis in transplanted mice?

\section{Hypothesis}

By reviewing previous literatures about HOXB4-mediated HSC expansion; Prdm 16's role in HSC maintenance; association of $s$ Prdm 16activation with leukemia in HOXB4 transplanted animals, we generated our hypothesis to answer the questions above. The hypothesis is:

HOXB4 downregulates Prdm16 to avoid leukemia in transplanted mice, cooverexpression of sPrdm16 and HOXB4 will lead to hematopoietic cell transformation.

\section{Rationales}

The Rationales of the hypothesis are listed as follows:

1. Overexpression of HOXB4 leads to murine HSC expansion without causing hematopoietic malignancies.

2. Activation of $s P r d m 16$ due to vector integration promotes leukemogenesis in HOXB4-overexpressing cells in large animals.

3. Growth of leukemia cells from HOXB4-transplanted animal is dependent on the $\operatorname{sidm} 16$ expression.

4. PRDM16 expression is upregulated in HOXA9-asscoated leukemias.

5. Aberrant expression of $s P R D M 16$ due to chromosome translocation or promoter hypomethylation is frequently detected in MDS/AML or adult T cell leukemias.

6. Overexpression of sPRDM16 led to myeloid leukemia in p53-null mice.

7. Enforced expression of $s$ Prdm 16 blocks G-CSF induced granulocytic differentiation in L-G3 cells.

\section{Specific aims}

1. Identify target genes and pathways regulated by HOXB4 in LSK cells from recipient mice at 6,12 and 20 weeks post transplantation.

2. Find out and validate the mechanism by which HOXB4 avoid leukemia in vivo. 


\section{Approach}

\section{Specific aim 1}

To identify HOXB4 transcriptional target genes and its regulated pathways we will perform gene expression profiling on HOXB4-overexpressing LSK cells from transplanted mice at 6,12 and 20 weeks following transplantation. A two-factor ANOVA analysis will be applied to identify differentially expressed target genes and Gene Set enrichment assay (GSEA) and MetaCore pathway analysis will be used to identify pathways regulated by HOXB4. Quantitative real-time PCR will be performed to validate differential gene changes in CD $34^{-/ \text {low }}$ LSK cells, which are highly enriched with HSCs.

Gene expression array provided by Hartwell Center for Bioinformatics and Biotechnology of St. Jude Children's Research Hospital requires at least 100ng mRNA with concentration of at least $33 \mathrm{ng} / \mathrm{ul}$. The major challenge of perform gene expression array on rare population is inadequate mount of RNA. In this study we plan to apply expression on LSK cells, which is about $0.3 \%$ of total bone marrow cells. Our HOXB4 retroviral vector can achieve about $30 \%$ transduction and around $90 \%$ for GFP vectors. Trial experiments showed that we are able to extract enough RNA for microarray from 35 mice.

\section{Specific aim 2}

To identify the mechanism by which HOXB4 avoids leukemia in transplanted animals we will screen the differentially regulated transcriptional target genes generated from microarray discussed above. They can be genes (1) critical for HSC differentiation or apoptosis, (2) tumor suppressors upregulated by HOXB4, (3) oncogenes downregulated by HOXB4. To validate the potential factor involved in avoiding leukemia, target gene will be overexpressed or knockdown using lentiviral vector under the context of HOXB4 overexpressing to screen leukemia in transplanted mice.

We have generated and well characterized lentiviral overexpression and knockdown vectors that can be used in this study. We also developed efficient HSC or HPC transduction protocol which allows us to generate enough double positive cells for transplantation. Flow cytometry core, Pathology department and Animal Resource Center of St. Jude Children's Research Hospital are able to offer us professional assistance for cell sorting and analysis, animal necropsy, animal care. 


\section{CHAPTER 2. IDENTIFICATION OF HOXB4 TRANSCRIPTIONAL TARGET GENES IN PRIMITIVE HEMATOPOIETIC CELLS IN VIVO}

\section{Introduction}

As mentioned earlier overexpression of HOXB4 leads to marked murine HSC expansion in vivo without causing hematopoietic malignancies. Little is known about the mechanism how HOXB4 expands HSCs and at meanwhile prevents leukemogenesis. To understand the regulatory role of HOXB4 in HSCs expansion, many studies were carried out to screen HOXB4's direct or indirect target genes and pathways important for HSCs maintenance, proliferation and differentiation. Microarray data from either enriched adult HSCs or embryonic derivatives expressing HOXB4 demonstrated that HOXB4 transcriptionally activates c-Myc ${ }^{249}$ and down-regulates genes involved in TNF- $\alpha$ and FGF signaling in bone marrow cells, ${ }^{250}$ thus proving beneficial for HSCs self-renewal. Gp49a, Laptm4b and Hemgn were identified as HOXB4 downstream targets and suggested to be potentially important for HSCs' stemness or expansion of myeloid progenitors in vitro. ${ }^{251,252}$ These earlier studies suggested that HOXB4 controls HSC or myeloid progenitors through multiple mechanisms such as apoptosis and symmetric division/proliferation. However, it is noticed that none of the previous studies focused on HOXB4 targets in primary primitive hematopoietic populations in vivo. To overcome the limits of previous screenings we decide to screen HOXB4's target genes in LSK cells during different phases of the reconstitution in vivo.

To screen HOXB4 target genes and pathways in LSK cells from transplanted mice, we plan to transplant HOXB4 overexpressing bone marrow cells to lethally irradiated mice and extract RNA of LSK cells from recipients at 6, 12 and 20 weeks following transplantation for expression array assay. Compared with previous studies done on manipulated cells lines or by using in vitro system, target genes identified from transplant mouse model will be more informative about how HOXB4 expands HSCs in vivo.

\section{Materials and Methods}

\section{Vector cloning}

The retroviral vectors MSCV-ires-GFP and MSCV-HOXB4-ires-YFP have been described previously. ${ }^{251}$

\section{Retroviral vector preparation}

Retroviral vectors of MSCV-ires-GFP or MSCV-HOXB4-ires-YFP were prepared as described before. ${ }^{251}$ The helper-free recombinant retroviral vector supernatant 
was freshly collected from the GPE +86 producer cells and filtered through $0.45 \mu \mathrm{m}$ pore filter for transduction.

\section{Bone marrow cells isolation, culture, transduction and transplantation}

All animal experiments were carried out according to protocols approved by the St. Jude Institutional Animal Care and Use committee. To harvest primitive bone marrow cells, 5-Fluorouracil (5-FU) (150 mg/kg) was injected intravenously into 6 to 8 -week-old female c57BL/6J mice. 5 days after injection bone marrow cells were flushed with 21 gauge needles from both femurs and tibias in to ice cold DPBS containing 2\% FBS. 5-FU treated were cultured in DMEM supplemented with 15\% fetal bovine serum (FBS), 50 units $/ \mathrm{ml}$ penicillin, 50 units $/ \mathrm{ml}$ streptomycin, $2 \mathrm{mM} \mathrm{L-glutamine,} 20 \mathrm{ng} / \mathrm{ml}$ recombinant mouse IL-3, $50 \mathrm{ng} / \mathrm{ml}$ human IL-6, and $50 \mathrm{ng} / \mathrm{ml}$ mouse SCF (R\&D Systems, Minneapolis, $\mathrm{MN})$.

To transduce bone marrow cells with gamma retroviral vector, 5-FU treated BM cells were prestimulated with cytokine mixture mentioned in the previous paragraph for 48 hours to induce quiescent hematopoietic stem cells to enter cell cycle. After two days' prestimulation $1 \times 10^{6}$ bone marrow cells were transduced with fresh unconcentrated supernatant from GPE+86-derived producer cells containing MSCV-HOXB4-ires-YFP or MSCV-ires-GFP retroviral vectors in 6-well plates preloaded with $25 \mathrm{ug} / \mathrm{ml}$ retronectin (Takara). After two to four daily exposures to vector, 1 to 2 million transduced cells were injected into the lateral tail vain of lethally irradiated (1100cGy) female C57B1/6L mice. Transplanted mice were under Baytril water administration for 3 weeks post transplantation and Baytril water was changed weekly.

\section{Cell separation}

BM cells were stained with Sca-1-PE, c-Kit-APC and a PE-Cy7 conjugated lineage antibody mixture (CD4, CD8, Gr1, Ter119 and NK1.1) (BD Bioscience). GFP ${ }^{+}$ or $\mathrm{YFP}^{+} \mathrm{LSK}$ cells were isolated by flow cytometry.

\section{Colony forming assay}

Total 500 vector positive LSK cells from HOXB4 or GFP transplanted mice at 12 and 20 weeks post transplantation were into $1 \mathrm{ml}$ MethoCult GF M3434 methylcellulose medium (StemCell Technologies, Vancouver, BC, Canada) containing various cytokines supporting growth of clonogenic myeloid progenitor cells. After 7 days, primary $\left(1^{\circ}\right)$ colonies were scored, collected, resuspended, and re-plated at $1 \times 10^{4}$ cells $/ \mathrm{ml}$ density into the M3434 methylcellulose medium for 14 days culture. The generated secondary $\left(2^{\circ}\right)$ colonies were scored to quantitate the expansion of myeloid progenitors. 


\section{Microarray expression analyses and real-time RT-PCR}

Total RNA from sorted LSK cells was extracted using RNAqueous ${ }^{\circledR}-$ Micro kit (Life Technologies). Approximately 20ng total RNA was processed using NuGEN WTA pico v2 and Encore Biotin labeling modules and 6ug of biotin-labeled cDNA was hybridized overnight to HT_MG-430_PM microarray chips (Affymetrix). Hybridized arrays were scanned using the Gene Titan system and signals were summarized using the RMA method (Partek Genomics Suite v6.5). Differentially expressed transcripts between GFP+ LSK and HOXB4+ LSK cells were identified from triplicate, independently derived from pools of cells at various time points using a two-factor ANOVA model. The Benjamini-Hochberg method ${ }^{253}$ was used to estimate the False Discovery Rate (FDR).

Pathways activated or repressed by HOXB4 expression were identified by GSEA ${ }^{254}$ using curated pathways obtained from the MolSigDB (Broad Institute) and Ingenuity Pathways (Ingenuity, Inc.). MetaCore ${ }^{\mathrm{TM}}$ software (GeneGo, Philadelphia, PA) was also used to identify significant biological pathways defined by specific differentially expressed genes.

To validate gene expression changes detected in microarray study, $\mathrm{GFP}^{+}$or HOXB4-YFP ${ }^{+} \mathrm{CD}^{-/ \text {low }}$ LSK cells were sorted from transplanted mice 20 weeks post transplantation. Total RNA sorted cells were reverse transcribed using SuperScript VILO cDNA Synthesis Kit (Life Technologies). Resulting cDNAs were quantitated by qRTPCR assay using Power SYBR ${ }^{\circledR}$ Green PCR Master Mix (Life Technologies). The relative expression levels for specific transcripts were calculated using the $2^{\Delta \Delta \mathrm{CT}}$ method normalized to the internal control gene Gapdh.

\section{Results}

\section{HOXB4 ${ }^{+}$LSK cells demonstrated enhanced hematopoietic progenitor cell self- renewal in vitro}

To demonstrate HOXB4-enhanced hematopoietic progenitors' clone forming capacity in vitro, $500 \mathrm{HOXB}_{-} \mathrm{YFP}^{+}$LSK cells or control GFP ${ }^{+}$LSK cells from 12 and 20 weeks transplant mice were plated into MethoCult GF M3434 to score the secondary colony numbers. HOXB-LSK from 12 week transplanted mice generated 169 secondary colonies, about 9 times more than GFP control LSK cells from the same time point $(\mathrm{P}<0.01)$ (Figure 2-1). HOXB4-LSK cells from 20 weeks transplant mice generated 395 secondary colonies, 15 times more than GFP control LSK cells at same time point $(\mathrm{P}<0.01)$ (Figure 2-1). Increased secondary colony number generated from HOXB4-LSK at both 12 and 20 weeks post transplantation indicated enhanced hematopoietic progenitor cell self-renewal in vitro. It also suggested RNA samples collected from the same batch of cells for microarray were validated. 


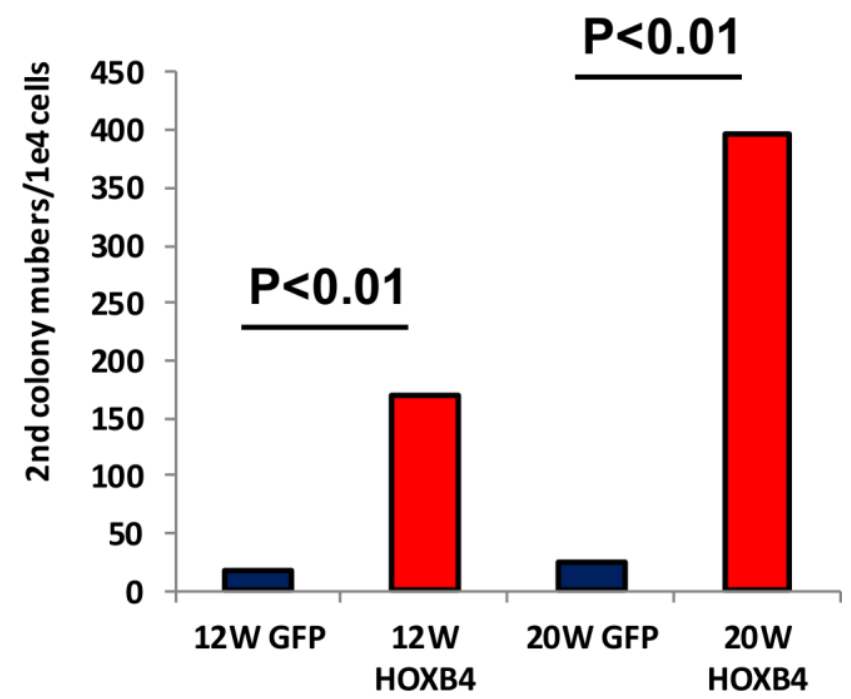

Figure 2-1. Secondary colony numbers generated from $1 \times 10^{4}$ GFP-LSK cells or HOXB4-LSK cells at 12 or 20 weeks post transplantation. 


\section{Global gene expression changes in HOXB4-transduced LSK cells at various time points after transplant}

To identify both direct and indirect transcriptional target genes of HOXB4 in HSCs, we generated a MSCV-HOXB4-ires-YFP vector and a control GFP vector based on the MSCV $\gamma$-retroviral vector (Figure 2-2A). Groups of mice were transplanted with transduced bone marrow cells and then euthanized at 6, 12, and 20 weeks after transplant for gene expression analysis (Figure 2-2B). Gene expression profiling was performed on HOXB4-YFP or control GFP-expressing bone marrow LSK cells isolated by cell sorting (Figure 2-2C). At most time points, sufficient amounts of RNA were isolated for microarray analysis (Table 2-1). For all time points, three independent transplant experiments and cell isolation procedures were performed to establish reproducibility.

Principal component analysis (PCA) using all expression profiles measured by the microarray revealed significant clustering within experimental groups (Figure 2-3A). The GFP samples and the HOXB4 samples separated across the first principal component indicating the most significant source of expression variation was associated with presence or absence of HOXB4 expression. Within the GFP treatment arm, the 12 and 20 week samples grouped closely together. Within the HOXB4 treatment arm, the 12 and 20 week samples also grouped together and were statistically distinct from the GFP-control groups (Figure 2-3A). The 6 week samples from the HOXB4 treatment arm were separated from the 12 and 20 week HOXB4 samples, demonstrating distinct transcriptional profiles exist at these two time points. A heat map representation of all expression profiles shows reproducible gene expression changes in HOXB4-transduced LSK cells that evolve between 6 and 20 weeks after transplant (Figure 2-3B). These gene expression patterns were consistent in triplicate samples from both groups and were highly reproducible within each experimental arm.

\section{Identification of specific changes in gene expression in HOXB4-transduced LSK cells}

To identify either direct or indirect HOXB4 target genes, we applied a two-factor ANOVA model to identify probe sets with $>0.5 \log$ difference and with a FDR of $<0.05$ in the HOXB4 versus the GFP groups at given time points. In the HOXB4-LSK cells, a total 520 and 1712 probe sets were identified with significant differential expression at 12 weeks and 20 weeks when compared with control GFP-LSK cells (Figure 2-4A, B). When 6 week HOXB4 samples were compared with the 12 and 20 week HOXB4 samples, 379 and 622 probe sets showed $>0.5 \mathrm{log}$ deference and FDR $<0.05$ respectively (Figure 2-4C, D). However, no significant differential gene expression was noted between 12 and 20 week in HOXB4 ${ }^{+}$LSK cells, demonstrating that the major transcriptional events in HOXB4 transduced cells occur by 12 weeks after transplantation. Heat maps of the top 35 over and under-expressed genes at 20 weeks show reproducible and significant differences in HOXB4samples versus GFP samples (Figure 2-4E). 
A.

MSCV-IRES-GFP

MSCV LTR ires GFP MSCV LTR

MSCV-HOXB4-IRES-YFP

MSCV LTR HOXB4 ires YFP MSCV LTR

B.

5-FU BMC

Injection isolation

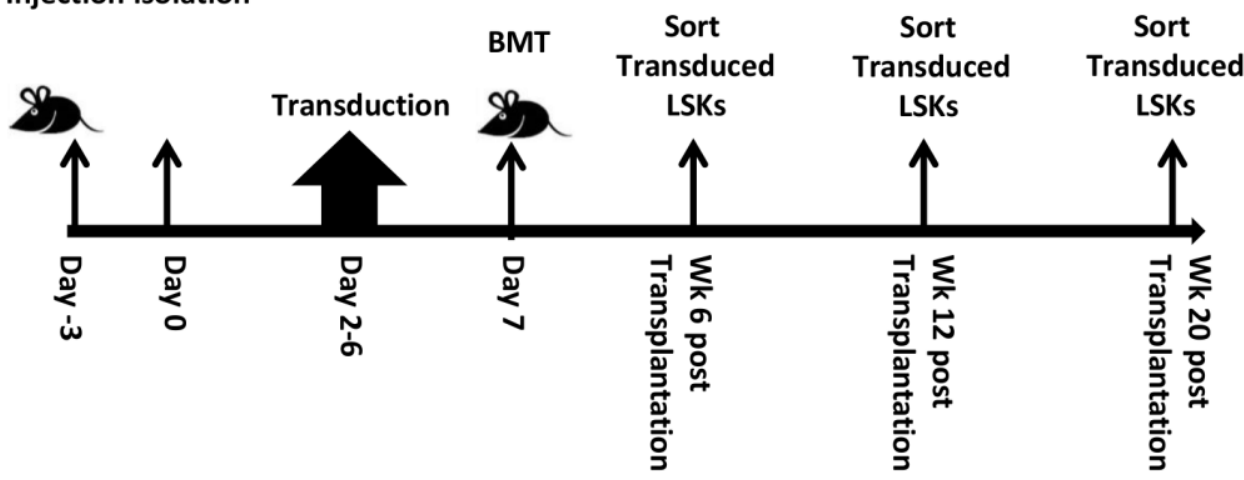

c.
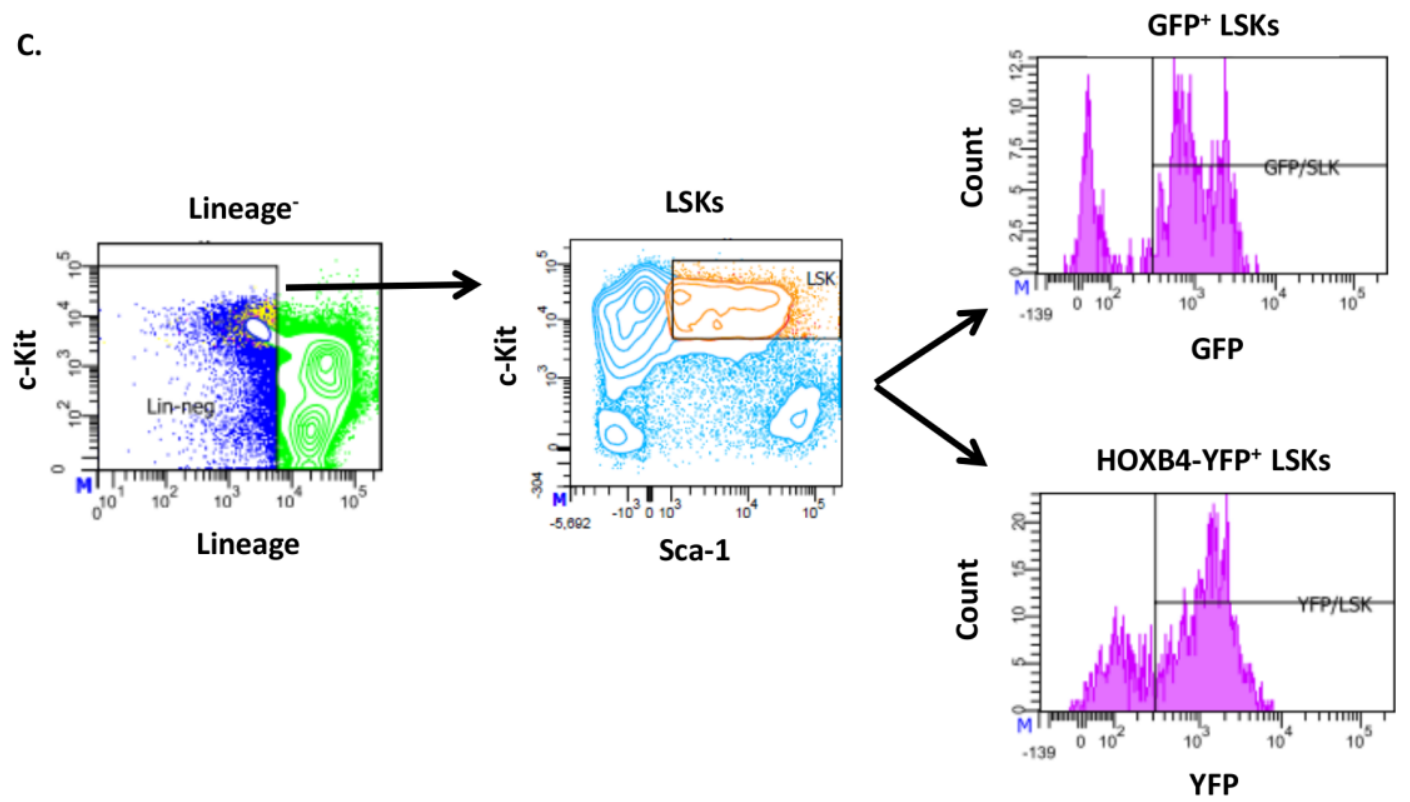

Figure 2-2. Experimental design of HOXB4 target gene analysis in transplanted murine HSCs.

(A) Schematic diagram of HOXB4 retroviral and GFP control vectors used to transduce 5-FU treated bone marrow cells. (B) Time line of transduction and mouse transplantation assay. (C) Flow cytometry sorting strategy for transduced LSKs. Bone marrow cells isolated from recipients were first gated for lineage negative cells, then for $\mathrm{cKit}^{+}, \mathrm{Sca}-1^{+}$ marker status, and then for vector expression based on GFP or YFP expression. Sorted GFP/YFP ${ }^{+}$LSK cells were used for subsequent gene expression analyses. 
Table 2-1. Summary of RNA samples submitted for microarray from GFP or HOXB4 group at 6 weeks, 12 weeks and 20 weeks.

\begin{tabular}{|c|c|c|c|c|}
\hline Group & Time Point & $\begin{array}{c}\text { Recipient } \\
\text { No. }\end{array}$ & LSK No. & $\begin{array}{c}\text { Amount of RNA } \\
\text { (ng) }\end{array}$ \\
\hline HOXB4 & 6 weeks & 2 & 12653 & $38.045^{*}$ \\
\hline HOXB4 & 6 weeks & 2 & 33599 & $51.2 *$ \\
\hline HOXB4 & 6 weeks & 4 & 178567 & $65.15^{*}$ \\
\hline GFP & 12 weeks & 2 & 103797 & $24.365^{*}$ \\
\hline GFP & 12 weeks & 2 & 132398 & $43.09 *$ \\
\hline GFP & 12 weeks & 3 & 32196 & $37.505^{*}$ \\
\hline HOXB4 & 12 weeks & 2 & 232034 & $153.7^{*}$ \\
\hline HOXB4 & 12 weeks & 2 & 145500 & $158.35^{*}$ \\
\hline HOXB4 & 12 weeks & 3 & 280349 & $209.8^{*}$ \\
\hline GFP & 20 weeks & 2 & 25532 & $33.782 *$ \\
\hline GFP & 20 weeks & 2 & 261449 & 221.4 \\
\hline GFP & 20 weeks & 2 & 11181 & $93.76^{*}$ \\
\hline HOXB4 & 20 weeks & 2 & 139257 & 172.54 \\
\hline HOXB4 & 20 weeks & 2 & 166132 & 252.2 \\
\hline HOXB4 & 20 weeks & 2 & 200269 & 411 \\
\hline
\end{tabular}


A.

PCA Mapping (44.1\%)

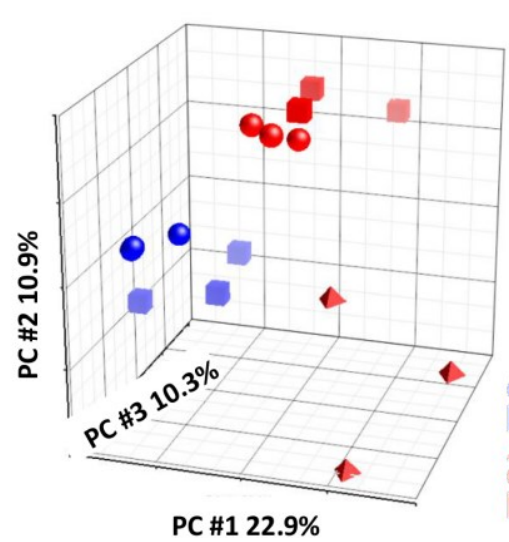

GFP 12Wk GFP 20Wk

HOXB4 6Wk

HOXB4 12Wk

HOXB4 20Wk
B.

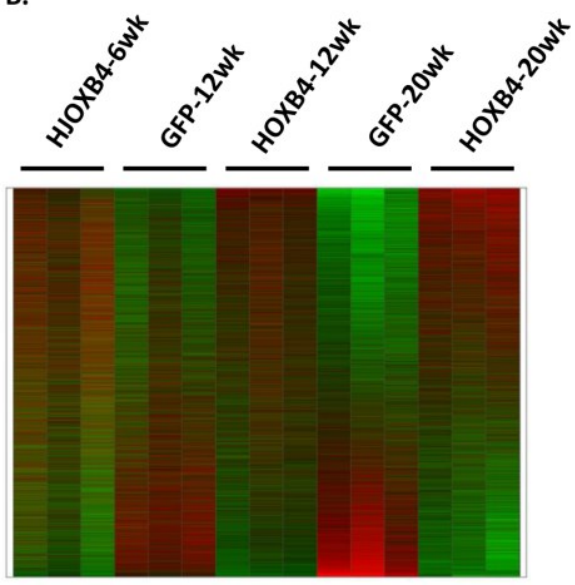

Figure 2-3. PCA and heat map representation of HOXB4-induced expression profiles in bone marrow LSK cells.

(A) PCA using all probe sets where each symbol represents an separate experiment as indicated by the legend, with red symbols denoting HOXB4 samples and blue symbols the GFP controls. The $\mathrm{GFP}^{+}$LSK cells and HOXB4 ${ }^{+}$LSK cells segregate into statistically separate groups across PC1 axis. HOXB4-LSK cells harvested at 6 weeks are distinct from 12wk and 20wk HOXB4-LSKs across the PC2 axis. (B) Heat map of gene expression alterations seen across all probe sets, with triplicate samples per experimental arm and time points as indicated by the labels. Red indicates over-expressed probe sets relative to GFP controls while green indicates under-expressed probe sets. 
A.

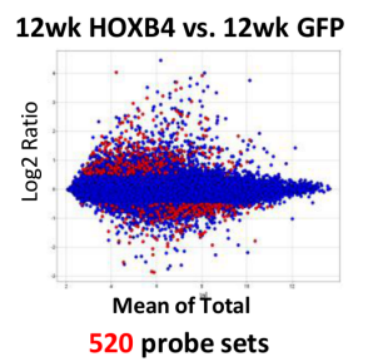

c.
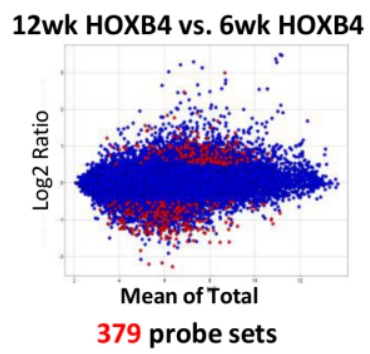

E.

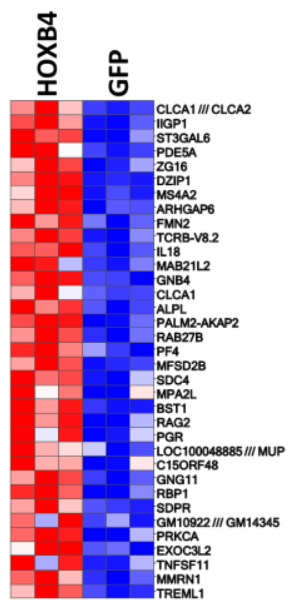

B.

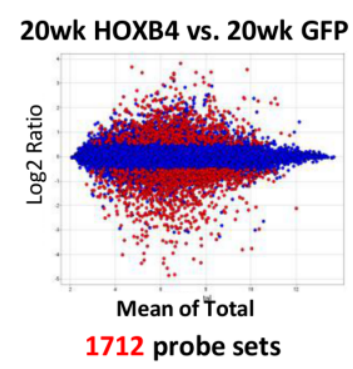

D.
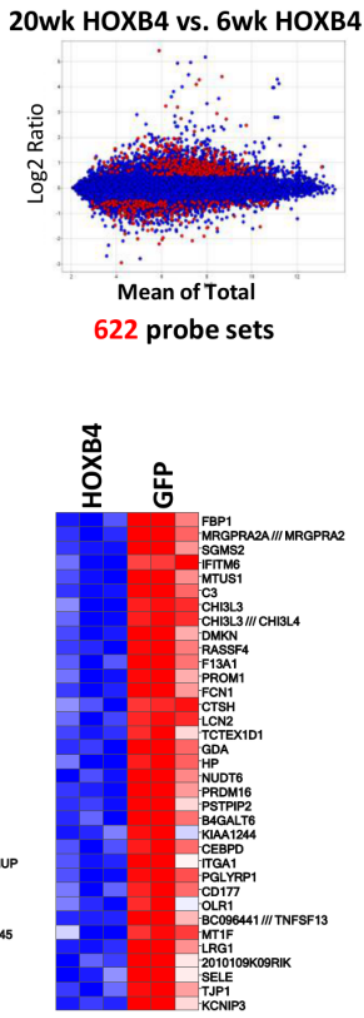

F.

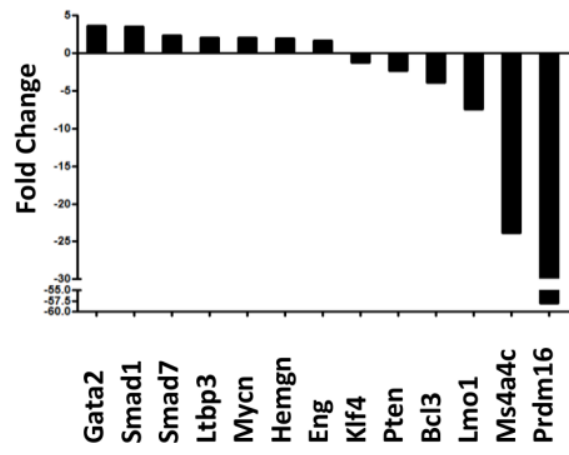

G

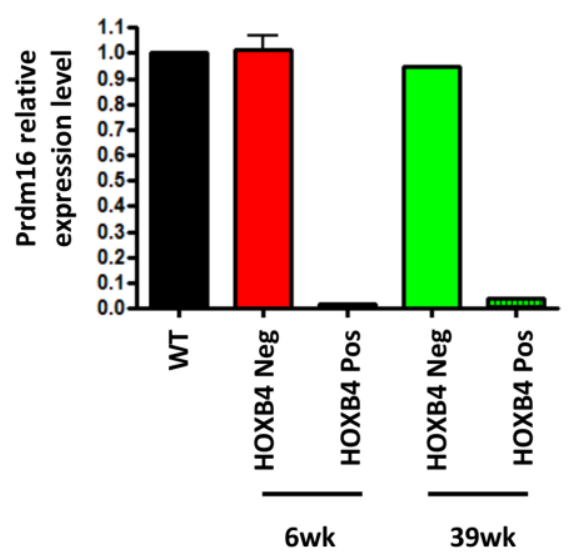

Figure 2-4. Identification and validation of HOXB4 target genes.

Bland-Altman plots showing comparative gene expression between (A) 12wk HOXB4LSKs and 12wk GFP-LSKs (B) 20wk HOXB4-LSKs and 20wk GFP-LSKs (C) 12wk HOXB4-LSKs and 6wk HOXB-LSKs and (D) 20wk HOXB4-LSKs and 6wk HOXB4LSKs. The $y$-axis indicates the magnitude of change and the $\mathrm{x}$-axis the average of the signal. The red dots represent differentially expressed probe sets with a $>0.5 \log$ differential expression and false discovery rate (FDR) $<0.05$. (E) Heat map of the top upregulated and downregulated genes from 20wk HOXB4-LSKs compared with 20wk GFP-LSKs. Red represents upregulated genes and blue boxes represent downregulated genes. (F) Quantitative RT-PCR measurement of differential gene expression for 13 HOXB4-regulated genes in CD34-/low LSK cells at 20 weeks post transplantation. The fold change is indicated for HOXB4 cells relative to GFP cells. (G) Quantitative RT-PCR for expression of Prdm 16 mRNA in LSK cells harvested from transplanted mice at 6 weeks and 39 weeks post transplantation. 
CD34 ${ }^{-/ \text {low }}$ LSK cells were isolated 20 weeks after transplant. Reverse qRT-PCR was used to measure mRNA levels for a subset of differentially expressed transcripts potentially involved in HSC regulation (Figure 2-4F). GATA2 is a zinc-finger transcription factor that is required for adult HSC development ${ }^{44}$ and was increased 4 fold in HOXB4 transduced HSCs. Hemgn was identified as a HOXB4 direct target in HOXB4 transduced hematopoietic progenitor cells in our previous study ${ }^{251}$ and was increased 3.0 fold relative to that seen in GFP control cells (Figure 2-4F). TGF-beta signaling is a negative regulator of HSC proliferation and utilizes SMAD proteins as downstream effector molecules ${ }^{84}$. Expression of Smad1 and Smad7 were 3.6 and 2.4 fold up-regulated by qRT-PCR in HOXB4-transduced CD34 ${ }^{-/ \text {low }}$ LSK cells (Figure 2-4F).

A 3-18 fold down-regulation of $\operatorname{Prdm} 16$ was noted in all 4 microarray probes from the HOXB4 samples at 20 weeks. PRDM16 is a SET domain-containing protein associated with epigenetic remodeling and is required for brown fat determination ${ }^{197,213,214}$ and adult murine HSC and neural stem cell maintenance. ${ }^{215} \mathrm{We}$ noted a 58.1 fold down-regulation of Prdm 16 mRNA by qRT-PCR in HOXB4 samples compared with GFP controls at 20 weeks (Figure 2-4F). A time course of Prdm 16 mRNA expression showed large decreases in Prdm 16 transcripts in HOXB4-LSK cells at 6 weeks and 39 weeks respectively (Figure 2-4G), demonstrating that downregulation of Prdm16 occurs at both early and late times after transplant.

\section{HOXB4 overexpression results in gene expression changes associated with HSC self- renewal and lymphoid-myeloid differentiation}

To further define the regulatory pathways underlying HOXB4-dependent HSC expansion, we performed GSEA with curated gene sets associated with signaling, metabolic, and differentiation pathways. HOXB4 expression at 20 weeks after transplant produced significant activation of genes associated with HSC function (Figure 2-5A). ${ }^{255}$ This trend was also seen at 12 weeks in the HOXB4 arm but did not reach statistical significance. Activation of genes associated with long term hematopoietic stem cell potential was also observed in HOXB4-transduced LSK cells at 20 weeks but not at 12 weeks (FDR < 0.05). The highest over-expressed genes in the HSC pathways included Jun, Evt1, Smad7, Angpt1, Laptm4b, Hoxa5, and Hoxa10 (Figure 2-5B).

The top canonical pathways activated and repressed in HOXB4-LSK cells at 20 weeks (Tables 2-2, 2-3) include multiple signaling, differentiation, and metabolic pathways. In particular, a distinct B cell differentiation pathway (Ingenuity, Inc.) was activated in HOXB4+ LSK cells at both 12 and 20 weeks (Figure 2-5C). Rag2, Rag1, Pax 5, Bcl11 a and other genes important for B cell differentiation were activated in HOXB4 ${ }^{+}$LSK cells (Figure 2-6A). Pathway analysis of differentially expressed transcripts using MetaCoreTM software identified several myeloid differentiation pathways as being activated by HOXB4 in LSK cells (Figure 2-5D). These included Development_Transcription regulation of granulocyte development and Development_GCSF-induced myeloid differentiation as the top two most significantly activated pathways in 12 and 20 weeks HOXB4-LSK cell samples (Figure 2-5D). Overexpressed genes in 
A.

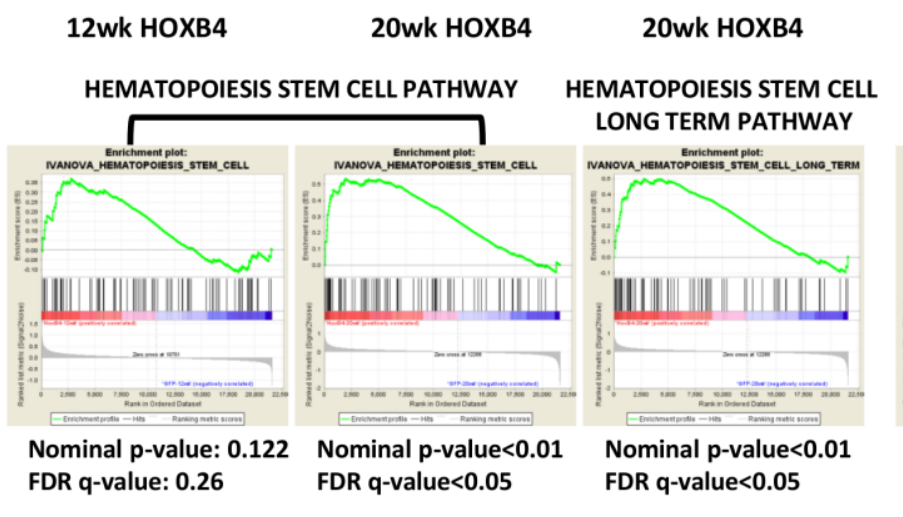

B.

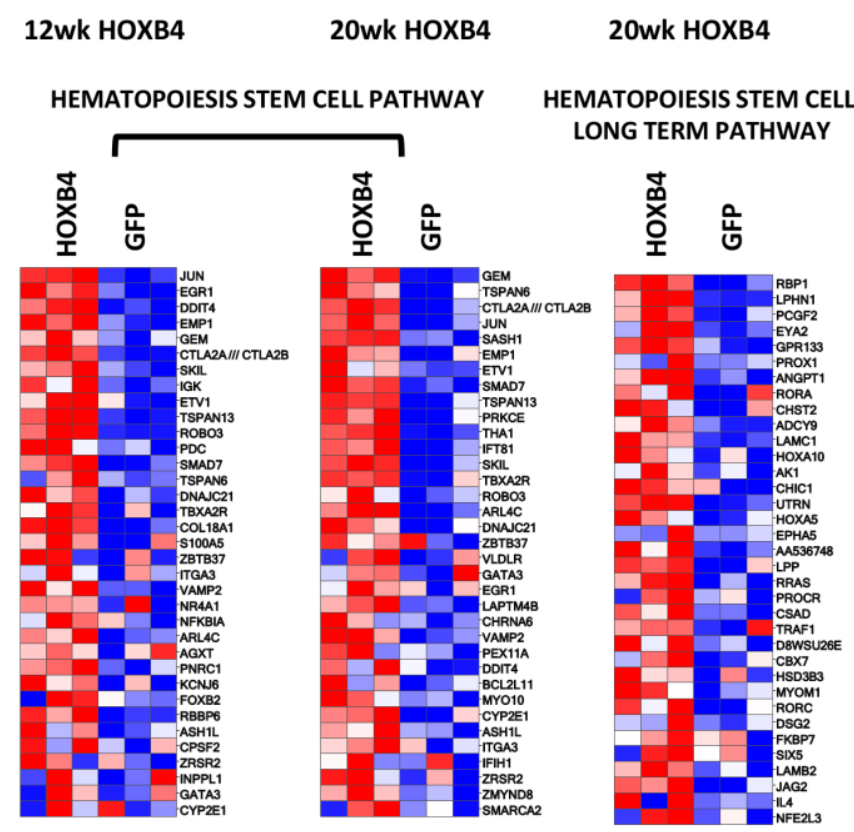

C.

12wk HOXB4 20wk HOXB4

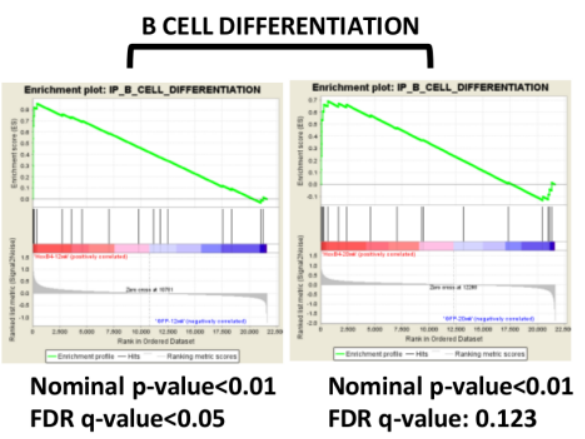

D.

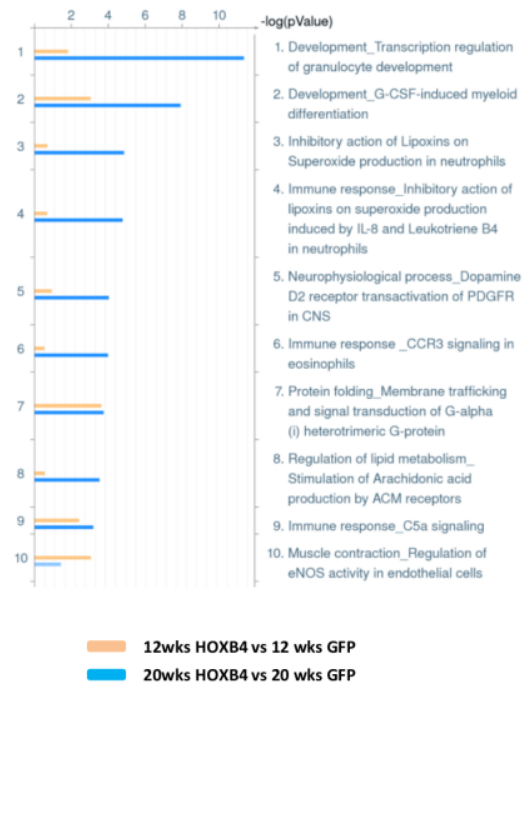

Figure 2-5. GSEA and MetaCore pathway analysis of HOXB4 responsive genes. (A) GSEA analyses for HOXB4 regulated genes identified for hematopoietic stem cell pathways. For each GSEA, the nominal p-value and FDR q-value are shown below each pathway graph. Statistical significance was validated for both pathways in 20 week samples. (B) Heat map gene expression changes in individual gene probe sets within the Hematopoiesis Stem Cell pathway and Hematopoiesis Stem Cell Long Term pathway in LSKs analyzed at 12 and 20wks after transplant. Red boxes represent upregulated genes and blue boxes represent downregulated genes. (C) GSEA analysis B cell differentiation pathway genes altered by HOXB4 in 12 and 20 week LSK samples. Statistical parameters are listed below each analysis. (D) Pathway analysis of differentially expressed probe sets using MetaCore ${ }^{\mathrm{TM}}$ program analysis showing the top 10 pathways regulated by HOXB4 at 12 (yellow) and 20 weeks (blue) post transplantation. The x-axis scale shows the $10 \log (\mathrm{p}$-value) of each pathway. 
Table 2-2. Pathways activated in HOXB4-LSKs at 20 weeks.

\begin{tabular}{|c|c|c|c|c|c|}
\hline Name & Size & $\mathbf{E S}$ & NES & NOM p-val & FDR q-val \\
\hline KEGG_PRIMARY_IMMUNODEFICIENCY & 35 & 0.67463 & 2.02402 & 0 & 0.013965 \\
\hline $\begin{array}{l}\text { REACTOME_THROMBIN_SIGNALING_THR } \\
\text { OUGH_RPOTINASE_ACTIVATED_RECEPT } \\
\text { ORS }\end{array}$ & 27 & 0.63399 & 1.84257 & 0.00168 & 0.16151 \\
\hline IP_B_CELL_DIFFERENTIATION & 20 & 0.690918 & 1.83225 & 0 & 0.12314 \\
\hline BIOCARTA_RHO_PATHWAY & 32 & 0.605903 & 1.82660 & 0.00543 & 0.102322 \\
\hline $\begin{array}{l}\text { KEGG_VASCULAR_SMOOTH_MUSCLE_CO } \\
\text { NTRACTION }\end{array}$ & 108 & 0.479403 & 1.82660 & 0 & 0.166082 \\
\hline BIOCARTA_BCR_PATHWAY & 33 & 0.59260 & 1.77393 & 0.00347 & 0.151108 \\
\hline BIOCARTA_PGC1A_PATHWAY & 22 & 0.64729 & 1.75932 & 0.00355 & 0.156613 \\
\hline $\begin{array}{l}\text { REACTOM_INHIBITION_OF_INSULINE_SE } \\
\text { CREATION_BY_ANDRENALINE_NORADR } \\
\text { ENALINE }\end{array}$ & 30 & 0.59687 & 1.74797 & 0.001694 & 0.159273 \\
\hline BIOCARTA_CTCF_PATHWAY & 23 & 0.62738 & 1.747828 & 0.005300 & 0.141576 \\
\hline REACTOME_SIGNAL_AMPLIFICATION & 31 & 0.582232 & 1.74742 & 0.003442 & 0.127858 \\
\hline
\end{tabular}


Table 2-3. Pathways activated in GFP-LSKs at 20 weeks.

\begin{tabular}{|c|c|c|c|c|c|}
\hline Name & Size & ES & NES & NOM p-val & FDR q-val \\
\hline KEGG_LYSOSOME & 116 & -0.59061 & -2.2849 & 0 & 0.00143257 \\
\hline $\begin{array}{l}\text { REACTOME_P53_INDEPENDENT_DNA_DAM } \\
\text { AGE_RESPONSE }\end{array}$ & 42 & -0.68597 & -2.2615 & 0 & $7.16 \mathrm{E}-04$ \\
\hline $\begin{array}{l}\text { REACTOME_CDT1_ASSOCIATION_WITH_TH } \\
\text { E_CDC6_ORC_ORIGIN_COMPLEX }\end{array}$ & 45 & -0.67419 & -2.2377 & 0 & 4.78E-04 \\
\hline KEGG_PROTEASOME & 44 & -0.67331 & -2.2255 & 0 & $7.14 \mathrm{E}-04$ \\
\hline $\begin{array}{l}\text { REACTOME_ELECTRON_TRANSPORT_CHAI } \\
\mathrm{N}\end{array}$ & 62 & -0.62747 & -2.2045 & 0 & 5.71E-04 \\
\hline $\begin{array}{l}\text { REACTOME_METABOLISM_OF_CARBOHYD } \\
\text { RATES }\end{array}$ & 110 & -0.5745 & -2.1961 & 0 & $4.76 \mathrm{E}-04$ \\
\hline REACTOME_STABILIZATION_OF_P53 & 45 & -0.6662 & -2.1781 & 0.002341 & 5.99E-04 \\
\hline $\begin{array}{l}\text { REACTOME_SCF_SKP2_MEDIATED_DEGRA } \\
\text { DATION_OF_P27_P21 }\end{array}$ & 50 & -0.6433 & -2.1659 & 0 & 8.47E-04 \\
\hline $\begin{array}{l}\text { REACTOME_CYCLIN_E_ASSOCIATED_EVEN } \\
\text { TS_DURING_G1_S_TRANSITION_ }\end{array}$ & 56 & -0.62507 & -2.1562 & 0 & $7.53 \mathrm{E}-04$ \\
\hline KEGG_PENTOSE_PHOSPHATE_PATHWAY & 26 & -0.7229 & -2.1551 & 0 & $6.78 \mathrm{E}-04$ \\
\hline
\end{tabular}


A.

B CELL DIFFERENTIATION PATHWAY

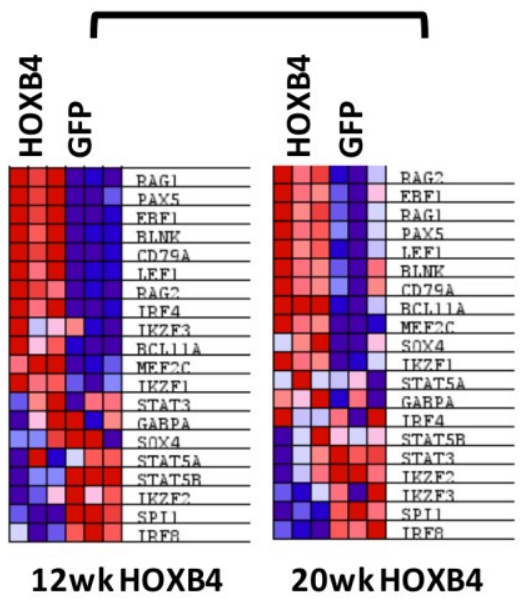

B.

Development_Transcriptional Regulation of granulocyte development

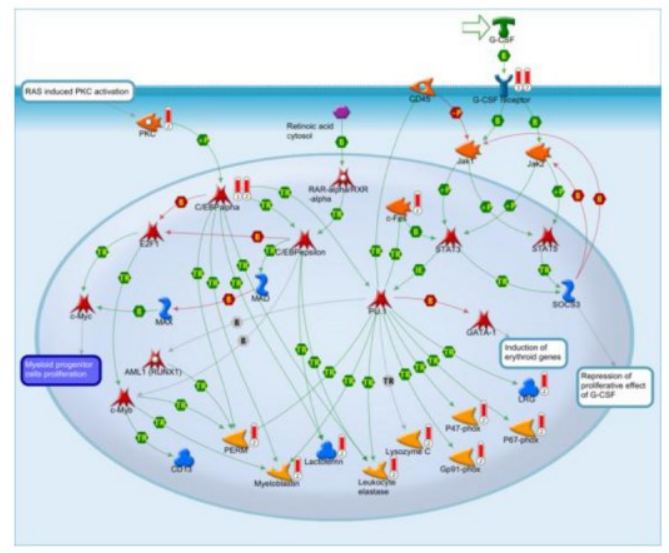

Development_G-CSF-induced myeloid differentiation

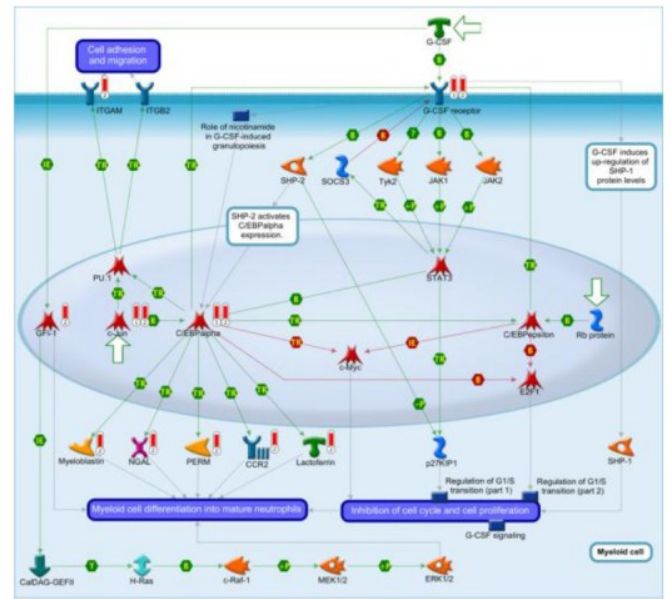

Figure 2-6. Activation of B cell differentiation and Granulocyte development/ GCSF-induced myeloid differentiation in 12wk and 20wk HOXB4-LSKs.

(A) Heat map of genes of B CELL DIFFERENTIATION pathway activated in $12 \mathrm{wk}$ and 20wk HOXB4-LKSs. (B) Demonstration of activated components in granulocyte development and G-CSF-induced myeloid differentiation signaling pathways in $12 \mathrm{wk}$ and 20wk HOXB4-LKSs. 
these pathways included genes encoding the G-CSF receptor as well as C/EBPalpha, Myeloblastin, Lactoferrin, Leukocyte elastase, and Gp91-phox (Figure 2-6B).

Altogether, these results suggest HOXB4's function may also include lineage-specific priming events in committed cells that are present within the heterogeneous LSK population.

\section{Discussion}

\section{Advantage of gene profiling study in LSK cells from HOXB4 transplanted mice}

Enforced expression of HOXB4 causes a controlled expansion of murine HSCs in vivo until HSC numbers approximate that seen in untransplanted mice. The genes that control this expansion have not been previously identified and are likely relevant for the physiologic regulation of HSC pool size or leukemogenesis in transplanted mice. Prior gene expression profiling studies have identified various HOXB4 target genes in different systems $^{250-252,256,257}$ yet these studies show little overlap in identified target genes. None of these previous studies included analyses of primary adult HSCs derived directly from HOXB4 transplanted animals. Even less is known about how HOXB4 transcriptional events are dynamically regulated at various times during HSC reconstitution. This study addresses these questions by defining the HOXB4 transcriptional program directly in LSK cells (HSC-enriched population) derived from the bone marrow of transplanted mice at multiple time points. Although majority of LSKs are composed of restricted progenitors, expression changes of genes critical for HSCs function were validated on CD $34^{-/ / \text {low }}$ LSK cells, which are high enriched with HSCs. Quantitative real-time PCR on CD $34^{-/ \text {low }}$ LSK cells confirmed the gene expression changes of key HSC regulators shown in gene expression array.

\section{Mechanism of well-maintained HSC pool size by HOXB4}

Our gene expression array data (PCA and heat map) showed that the HOXB4 gene expression pattern in transplanted LSK cells was fully established by 12 weeks after transplant. This is consistent with the known kinetics of HOXB4-induced HSC expansion in which HSC numbers in the bone marrow were normal and stable by 12 weeks after transplant, but not at 8 weeks. ${ }^{181,182}$ The fact that the kinetics of the gene expression changes correlate with the plateauing of HSC numbers further establish that HOXB4 mediated HSC effects are transcriptionally mediated. GSEA showed that both short and long term HSC expression profiles were activated by HOXB4 expression and involved regulation of a number of genes known to be important for HSC function and selfrenewal.

When taking close look at the genes critical for HSC proliferation, quiescence and apoptosis, Smads, Gata2, Bcl3 and Prdm 16 would be executors which regulate HSC pool size in HOXB4 transplanted mice. SMAD-1 (R-Smad) and SMAD-7 (I-Smad) are 
mediators of TGF- $\beta$ /BMP signaling pathway, which execute ligand-specific regulation of HSC proliferation, differentiation and apoptosis and in some cases bone marrow niche is required. Smad1 and Smad7 were upregulated in HOXB4-LSK/CD34-/low LSK cells. Smad7 increases HSC self-renewal in vivo while effect of Smadl on adult HSC is not clear, although Smad1 was required for embryonic hematopoiesis. Considering Smad1 is activated by TGF- $\beta 1$ receptor which has negative effect on HSC self-renewal and differentiation and promotes HSC apoptosis, we assume that activation of Smad1 in HOXB4 transplanted mice had negative regulation of HSC pool size. The ultimate outcome is decided by the balanced effect of Smad1 and Smad7. GATA2, a transcription factor inhibiting hematopoietic precursor from differentiation and maintaining HSC/HPC at quiescent state, ${ }^{45,46}$ was upregulated by HOXB4 in LSK cells. It suggests GATA2's role in maintaining HSC pool size. Another mechanism for HOXB4 to regulate HSC pool size is downregulation of $B c l 3$. BCL3 is a potential anti-apoptotic effector in HSCs and repression of $\mathrm{Bcl} 3$ results in increased apoptosis in HSCs, thus prevent HSC 'runaway' expansion. PRDM16 is another positive regulator of HSC which was marked downregulated by HOXB4. Prdm 16 null mice are embryonic lethal because of loss of fetal liver HSCs due to enhanced apoptosis. ${ }^{215,216}$ Repression of Prdm16 expression can introduce apoptosis in HOXB4-LSKs, thus maintain HSC pool at physiological level. (Figure 2-7)

In addition to these HSC signatures, network analyses revealed gene sets enrichment associated with B-cell and myeloid differentiation programs. Activation of these differentiation programs could reflect expression of lineage-associated genes that result from asymmetric HSC divisions producing committed progenitor cells. This is consistent with the widely held viewpoint that HSC pool size is regulated by establishing equilibrium between commitment/differentiation versus HSC self-renewal fates. The LSK cell pool contains seven functionally distinct subpopulations of HSCs and MPPs with myeloid-biased or lymphoid-biased or myeloid-lymphoid balanced reconstitution capacity in transplanted mice. ${ }^{258}$ Our data suggest that HOXB4 overexpression can induce both symmetric and asymmetric HSC replication in a balanced manner that leads to a stable HSC pool size by 12 weeks.

To validate the hypothesis of the regulation of HSC pool size in HOXB4 transplanted mice discussed above, limiting dilution assay to assess the HSC frequency should be performed.

\section{Mechanism how HOXB4 prevents leukemia in transplanted mice}

Overexpression of HOXA9 or HOXA10 can lead to myeloid leukemia because of increased HSC/HPC pool size and blocked downstream differentiation, during which collaboration of MEIS1 is required. ${ }^{138-140}$ It is known that HOXB4, unlike oncogenic gene HOXA9 and HOXA10, doesn't cause hematopoietic malignancies in transplanted mice. Differentiation permission of proliferating HSCs is the key to prevent leukemia. GSEA and MetaCore pathway analysis indicate HOXB4 activates B cell and myeloid lineage differentiation, thus preventing accumulation of undifferentiated HSCs in bone 


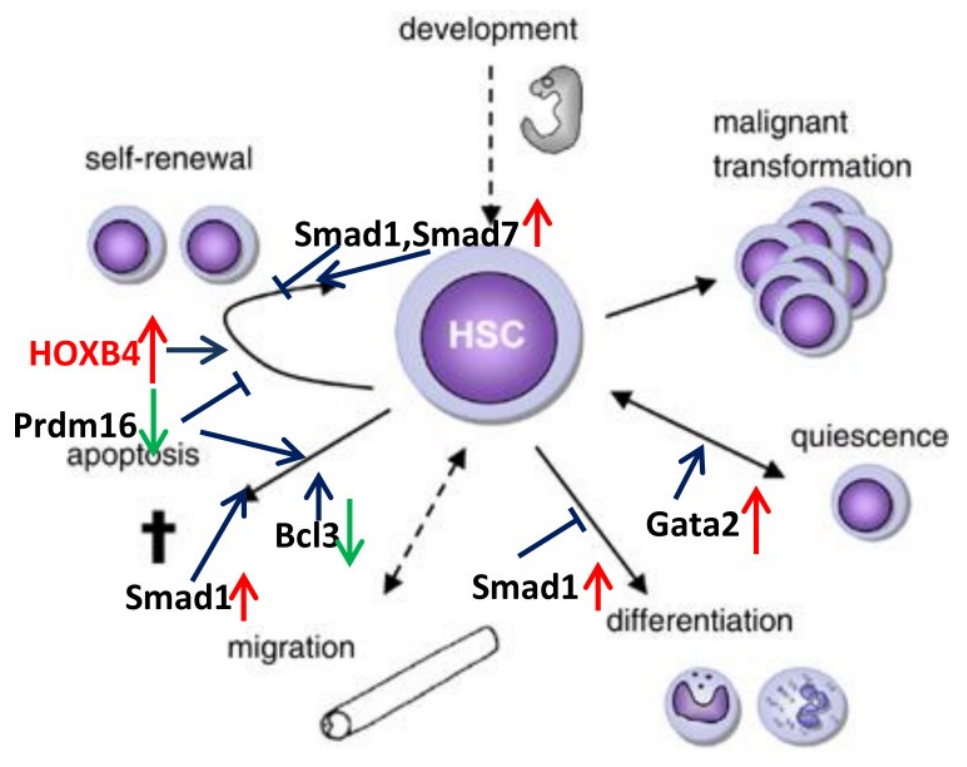

Figure 2-7. Regulation of HSC pool size in HOXB4 transplanted mice. Genes known important for HSC function, Smad1, Smad7, Gata2, Bcl3 and Prdm16, were regulated to maintain HSC pool size at physiological level. Maintenance of HSC pool size was achieved through regulation of HSC self-renewal, differentiation and apoptosis.

Modified with permission. Larsson J, Karlsson S. The role of Smad signaling in hematopoiesis. Oncogene. 2005;24(37):5676-5692. ${ }^{12}$ 
marrow niche which could potentially lead to leukemia. However the molecular mechanism of the differentiation permission in HOXB4 expressing HSCs is not clear. PRDM16 as mentioned before is required for HSC maintenance duringembryogenesis ${ }^{215,216}$ and its aberrant expression is frequently detected in MDS/AML or T-ALL patients. ${ }^{223-225,237-239,247,259,260}$ Downregulation of Prdm16 by HOXB4 can serve a mechanism to prevent leukemia in transplanted mice. Due to chromosome translocation or vector insertional activation, short form of PRDM16 (sPRDM16) lacking most of the PR domain is the major isoform expressed in leukemia patients. Overexpression of sPRDM16 but not full length of PRDM16 led to myeloid leukemia in $p 53$ null mice. ${ }^{248}$ To prove the hypothesis that repression of Prdm16 is the mechanism to prevent leukemogenesis in HOXB4 transplanted mice, we plan to screen leukemia in transplanted mice receiving HOXB4 and sPRDM16 co-expressing cells in mice. We expect to detect myeloid leukemia in transplanted mice. It may require long latency or we will not detect any leukemia, which suggests additional genetic lesions are required to initiate leukemic transformation. 


\section{CHAPTER 3. DOWNREGULATION OF PRDM16 PREVENTS LEUKEMIA IN HOXB4 TRANSPLANTED MICE}

\section{Introduction}

Enforced expression of HOXB4 leads to increased HSC self-renewal without causing hematopoietic malignancies in transplanted mice ${ }^{141,181}$. Benign HSC expansion in vivo makes HOXB4 a perfect tool to expand HSCs for blood diseases. To identify the mechanism how HOXB4 prevents leukemogenesis, we investigated the expression profiling of HOXB4 expressing LSKs from transplanted, which suggested activated lineage priming and downregulation of AML-associated gene Prdm16 would be the reason. We focused on the study of $s \operatorname{Prdm} 16$ because of the association of sPrdm16 with human MDS/AML and T-TALL. ${ }^{239,247,260}$ Humphries group also reported the vector insertional activation of sPrdm 16 which plays role in the myeloid leukemia developed in large animals transplanted with HOXB4 transduced CD34+ ${ }^{+}$cells. ${ }^{187}$ At meanwhile Forget's group screened 52 HOXB4 transplanted mice and 2 mice developed AML at 4 and 9.5 months, respectively, following transplantation. Both leukemias were passaged by serial transplantation of leukemic bone marrow or spleen cells into WT recipient mice. Leukemia 1(L1) showed expression of Mac-1 and Gr-1 myeloid markers as well as CD16/32 and Sca1 (Figure 3-1A) and the second leukemia case (L2) demonstrated expression of myeloid (Gr-1, Mac-1), erythroid (Ter119), and B lymphoid (B220) markers (Figure 3-1B). Interestingly, identification of vector insertion sites revealed an intragenic integration in intron 1 of the $\operatorname{Prdm} 16$ gene and another integration event approximately 50kb upstream of Notch1 gene (Figure 3-2A). Increased levels of sPrdm 16 and Notch 1 mRNA were confirmed in L2 leukemia cells (Figure 3-2B). Activation of sPrdm16 in large animals and mice suggested sPrdm16's role in initiation or maintenance of leukemia in HOXB4 transplanted animals. However, we do not exclude other molecular changes which also can contribute to the leukemic transformation, because other pro-oncogenes were also activated by vector integration together with sPrdm16. In the following studies we decided to focus on $\operatorname{srdm} 16$ to initiate the further exploration of the mechanism how HOXB4 avoids leukemia in transplanted mice.

Building on our gene expression array data, independent leukemia screening by Forget's group and previous literature reports, we generated the hypothesis that downregulation of Prdm 16 is the specific mechanism for HOXB4 to prevent leukemia in transplanted mice. To test the hypothesis we overexpressed HOXB4 and sPrdm 16 in hematopoietic primitive cells and screen leukemia in the transplanted mice receiving the co-expressing cells. If repression of Prdm 16 serves as a major factor limiting hematopoietic cell transformation, we expect to detect leukemia developed from HOXB4 and SPRDM16 co-overexpressing cells. Leukemias to be detected are most likely myeloid leukemias. We do not expect leukemia in sPRDM16 only group because both p53 loss and $s$ Prdm 16 activation are required for leukemogenesis. ${ }^{248}$ If we did not detect any leukemia, it can due to the following reasons. (1) Additional genetic lesions are required for leukemic transformation. (2) sPrdm 16 overexpression vector induces an inappropriate 
A. L1

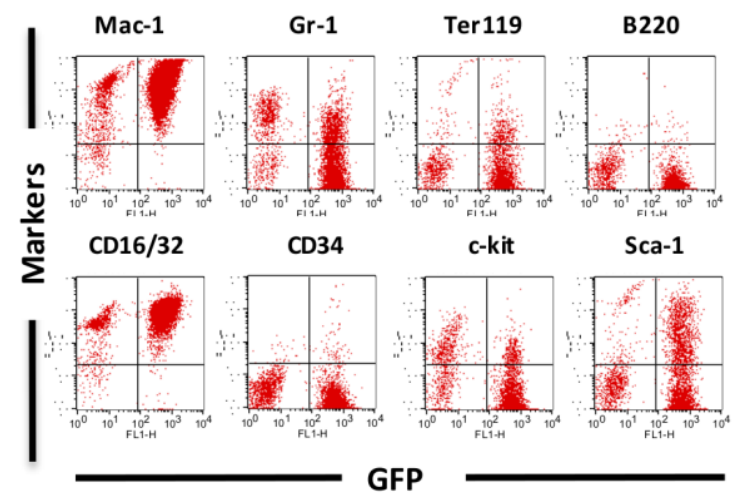

B. L2

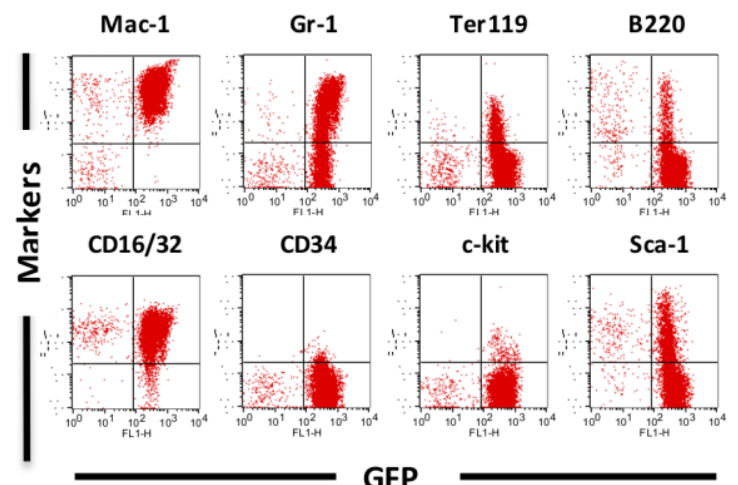

Figure 3-1. HOXB4-mediated leukemia in transplanted mice.

(A) Immunophenotying of bone marrow cells from recipients displaying leukemia cases L1 and L2. The y-axis shows the indicated cell surface markers identified by antibody staining and the x-axis shows GFP expression associated with the HOXB4 vector. Expression of mono-myeloid cell surface markers (Mac-1, CD16/32, Gr-1) and also erythroid (Ter119) and B lymphoid (B220) markers are shown. A smaller percentage of the leukemic cells also expressed the stem cell markers c-Kit and Sca1. (B) Retroviral insertion sites in L2 were identified by splinkerette PCR. The location of the 2 insertion sites in the adjacent to Notch1 and within Prdm16 and shown schematically along with the orientation of the vector at the insertion site. 
A.

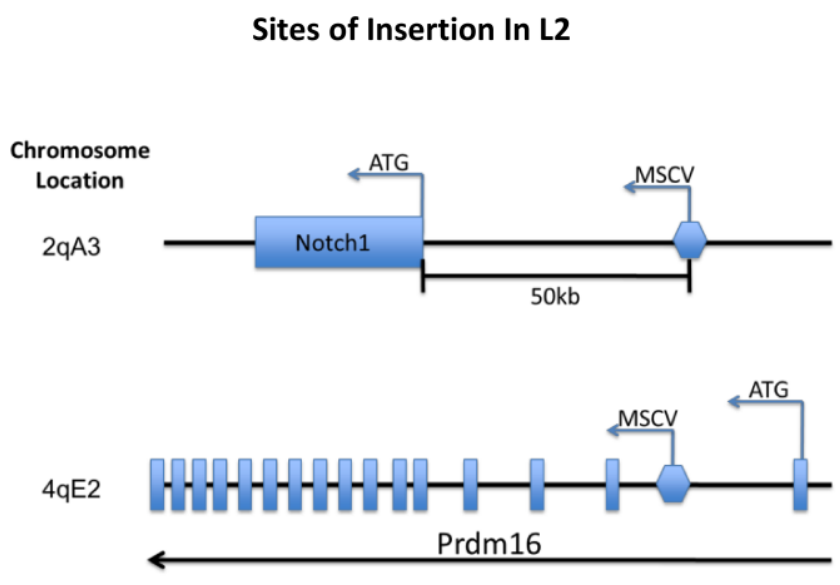

B.

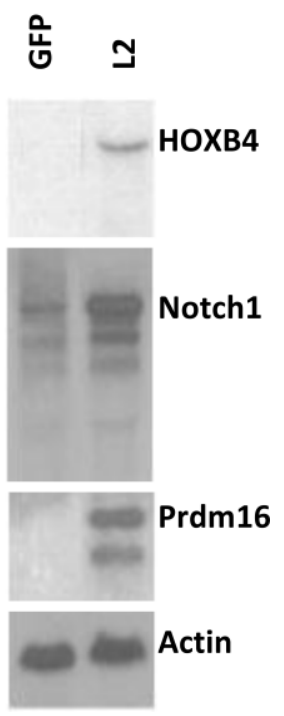

Figure 3-2. Vector insertion site-mediated oncogene activation in HOXB4mediated leukemia 2 in transplanted mice.

(A) Retroviral insertion sites in L2 identified by splinkerette PCR. The location of the 4 insertion sites were identified in intervening sequences and 6 insertion sites were between genes. (B) Northern blot analysis of relevant oncogenic mRNAs from control and HOXB4 leukemic cells from L2. The control cells consisted of bone marrow cells of mice transplanted with normal BM cells transduced with the "empty" MSCV/GFP vector; lane labeled GFP. There is a markedly increased level of Prdm16 mRNA and a mild increase ( 2 fold) of Notch1 mRNA in L2 BMCs compared to control BMCs. Actin serves as an internal loading control. 
overexpression level of sPrdm16 or (3) repression of sPrdm16 is not the reason why HOXB4 doesn't cause leukemia in transplanted mice.

\section{Materials and Methods}

\section{Vector cloning}

The retroviral vectors MSCV-HA-HOXA9-ires-GFP and MSCV-HOXA10-iresGFP were kindly provided by Dr. Keith Humphries (Terry Fox Laboratory, Vancouver, Canada).

Murine sPrdm16 cDNA (nucleotide 664-3822 of Mus musculus Prdm16 transcript, NM_00117795) was generated by PCR using a mouse Prdm16 cDNA (Thermo, Clone ID 6409778) as template. The sPrdm16 cDNA was inserted into the CL20 MSCV-ires-mCherry lentiviral vector using EcoRI site to generate the CL20 MSCV-sPrdm16-ires-mCherry vector. The CL20 MSCV-ires-mCherry vector was derived originally from pCL20c MSCV-GFP vector. ${ }^{23}$

The CL20i4r-EF1 $\alpha$-HOXB4-P2A-GFP lentiviral vector was generated by PCR using the MSCV-HOXB4-ires-YFP retroviral vector as template. An EcoRI-HOXB4P2A-GFP-NotI fragment was generated by PCR and was digested with EcoRI and NotI

and used to replace the hgcOPT fragment in CL20i4r-EF1 $\alpha$-hgcOPT lentiviral vector ${ }^{261}$

To generate MSCV-HOXB4-ires-mCherry vector, HOXB4 cDNA was released from MSCV-HOXB-ires-YFP retro viral vector using EcoRI site and ligated into MSCVires-mCherry lentiviral backbone between MSCV LTR and Internal ribosome entry site (IRES) fragment.

\section{Retroviral vector preparation}

A polyclonal ecotropic retroviral producer GPE+86 cells of MSCV-HA-HOXA9ires-GFP and MSCV-HOXA10-ires-GFP was generated using calcium phosphate transient transfection. $1 \mu \mathrm{g}$ pCAGGS-VSVG and $5 \mu \mathrm{g}$ old-gap-pol helper plasmids were co-transfected with $10 \mu \mathrm{g}$ MSCV-HA-HOXA9-ires-GFP or MSCV-HOXA10-ires-GFP vector into $10 \times 10^{6}$ cycling $293 \mathrm{~T}$ cells using $50 \mathrm{ul} 2.5 \mathrm{M}$ calcium chloride $\left(\mathrm{CaCl}_{2}\right)$ and $1 \mathrm{x}$ HBSS. Retroviral vector supernatant was harvested 48 hours post transfection, pooled, filtered through $0.45 \mu \mathrm{m}$ PVDF filter (Millipore, Billerica, MA), and used to transduce the cycling GPE +86 cells in the presence of $5 \mu \mathrm{g} / \mathrm{ml}$ protamine. The transduced GFP positive GPE +86 cells were sorted and expanded in culture to establish stable retroviral vector producer lines. 


\section{Calcium phosphate transfection and vector concentration for lentiviral vector}

Six hours before transient transfection approximately 10x $10^{6}$ HEK293T cells were seeded in 10-cm non-treated plates in 10ml DMEM supplemented with 10\% FBS, 50 units $/ \mathrm{ml}$ penicillion, 50 units $/ \mathrm{ml}$ streptomycine and $2 \mathrm{mM}$ L-glutamine. For production of 10 plates of lentiviral vectors 10ug transgene containing vector, 60ug CAG-Kgp1-1, 20ug pCAGG-VSVG and 20ug CAG-RTR2 were mixed with water and $500 \mathrm{ul} 2.5 \mathrm{M} \mathrm{CaCl}_{2}$ up to $5 \mathrm{ml}$ final volume. $2 \mathrm{x}$ HBSS was added to $\mathrm{DNA}-\mathrm{CaCl}_{2}$ mix drop wise while vortexing. $1 \mathrm{ml}$ DNA- $\mathrm{CaCl}_{2}$-HBSS mix was added into to each HEK293T plate and cells were incubated at $37^{\circ} \mathrm{C}$ in $5 \%$ carbon dioxide. Medium was changed with $8 \mathrm{ml} \mathrm{X}$-vivo-10 for each plate 18 hours after transfection and supernatant containing lentiviral vector was harvested 42 hours post transfection. Supernatant was filtered with $0.45 \mathrm{um}$ low protein binding filter (Millipore, Billerica, MA). One day before vector concentration Beckman Coulter SW28 rotor, swinging buckets with centrifuge tube adaptors and rotor tubes were kept at $4^{\circ} \mathrm{C}$. Centrifugation tubes were sprayed with $70 \%$ ethanol and $30 \mathrm{ml}$ of the virus supernatant was added into centrifuge tube. Supernatant was spin at $25 \mathrm{~K} \mathrm{rpm}$ at $4^{\circ} \mathrm{C}$ for 90 minutes. As soon as spin was over, supernatant was removed and 250ul X-vivo-10 was added to each centrifugation tube. And centrifuge tubes were kept on ice overnight. Next day lentiviral particles were collected, aliquot into certain volume desired and stored at $-80^{\circ} \mathrm{C}$.

\section{Bone marrow cells isolation, culture and transduction}

Harvest and retroviral transduction of 5-FU treatment bone marrow cells were carried out as described in Chapter 2. 5-FU treated bone marrow cells were transduced with MSCV-ires-GFP, MSCV-HOXB4-ires-YFP, MSCV-HA-HOX9-ires-GFP or MSCV-HOXA10-ires-GFP retroviral vector, respectively.

To harvest lineage-depleted bone marrow cells, whole bone marrow cells were isolated from 6 to 8 week old c57BL/6J mice and Lineage positive cells were depleted with Lineage Cell Depletion Kit, Mouse (Miltenyl Biotec) and LS columns (Miltenyl Biotec) according to manufacture instructions.

To co-overexpress $s P r d m 16$ and $H O X B 4$, lineage-depleted bone marrow cells from 6-8 weeks-old female $\mathrm{C} 57 \mathrm{Bl} / 6 \mathrm{~L}$ mice were transduced with concentrated CL20i4rEF1 $\alpha$-HOXB4-P2A-GFP and CL20-MSCV-sPrdm16-ires-mCherry vectors concurrently for 4 continuous days. Transduced bone marrow cells were subjected to colony forming assay and transplantation assay described in following paragraphs.

\section{In vitro expansion assay}

After transduction, transduction efficiency was evaluated by flow cytometry 2 days post last transduction. The transduction efficiencies were about $50 \%$ for GFP control vector, $10-30 \%$ for HOXB4 vector, $20-50 \%$ for HOXA9 vector and $20-30 \%$ for 
HOXA10 vector. For liquid culture and CFU-C assays, cells were diluted with nontransduced cells to achieve the similar GFP percentage (about 10\%), and then $5 \times 10^{5}$ normalized cells were cultured in cytokine-supplemented medium. YFP or GFP percentage was determined by flow cytometry and cells were counted every 3 days during the culture to evaluate growth advantage. 500 normalized cells cultured in M3434 methylcellulose medium for colony forming assay. Colony replating and colony scoring were described in Chapter 2.

For HOXB4 and sPrdm 16 co-overexprssion experiment fluorescence-activated cell sorting (FACS) was used to sort HOXB4 and sPRDM16 double positive cells, which were subjected into colony forming assay in vitro. Plating of cells and colony scoring were described in chapter 2 .

\section{Transplantation assay}

To evaluate Prdm 16 expression level in $\mathrm{GFP}^{+}, \mathrm{HOXB}^{+}, \mathrm{HOXA}^{+}$and $\mathrm{HOXA10}{ }^{+}$ primitive bone marrow cells at 6 weeks post transplantation, transplantation of transduced bone marrow cells were conducted as described in Chapter 2.

To screen leukemia in HOXB4 and sPrdm 16 co-expressing bone marrow cells $5 \times 10^{5}$ or $1 \times 10^{6}$ unsorted co-transduced bone marrow cells were injected into lethally irradiated B6.SJL-PtprcaPepcb/BoyJ mice (Jackson Lab, stock number: 002014).

\section{Fluorescence-activated cell sorting}

To isolate each compartments of BM cells, whole BM cells were stained with different antibody cocktails. For CD $34^{-/ \text {low }}$ LSK cells, CD $34^{\text {high }}$ LSK cells, CMP, GMP and MEP, cell were stained with CD34-PE, Sca-1-Percp5.5, c-Kit-APC, FceR-Alexa700 and a PE-Cy7 conjugated lineage antibody mixture (CD4, CD8, Gr1, Ter119 and NK1.1) (BD Bioscience). To separate mature BM cells from each lineage, cells were stained with Gr1-APC-Cy7, Mac1-Alexa700, B220-FITC, CD3-APC, NK1.1-PE, and Ter119-PE-Cy7 (BD Bioscience).

To isolate LSK cells, staining was carried out in the same way described in Chapter 2.

To isolate vector positive $\mathrm{CD} 150^{+} \mathrm{CD} 48^{-} \mathrm{LSKs}, \mathrm{CD} 150^{+} \mathrm{CD} 48^{+} \mathrm{LSKs}$ and $\mathrm{CD} 150^{-}$ CD48 ${ }^{+}$LSKs from GFP, HOXB4, HOXA9 or HOXA10 transplanted mice, bone marrow cells from recipients were stain with Sca1-PE (BD Bioscience) and PE-conjugated microbeads (Miltenyl Biotec). Sca1-PE positive cells were selected by LS column (Miltenyl Biotec) and further stained with CD150-PE-Cy7, CD48-APC, c-kit-APC-e780 and lineage cocktail-e605 antibodies (BD Bioscience). 
Stained cells were subject to FACS and each population was identified and sorted according to isotype and single color controls.

\section{Real-time PCR assay}

Total RNA extraction, reverse transcription and qRT-PCR assay were conducted as described in Chapter 2.

\section{Western blot}

Total protein lysate extraction of HOXB4 or $s$ Prdm 16 transduced NIH-3T3 cells and western blot were carried out as described ${ }^{28}$. HOXB4 protein was probed using $0.1 \mathrm{ug} / \mathrm{ml}$ I12 rat anti-mouse HOXB4 antibody (Developmental Studies Hybridoma Bank, University of Iowa) followed by HRP-conjugated goat anti-rat secondary antibody (Santa Cruz Biotechnology) and sPRDM16 protein was probed with $0.1 \mathrm{ug} / \mathrm{ml}$ sheep anti-mouse PRDM16 polyclonal antibody (R\&D systems) followed by HRP-conjugated donkey antisheep secondary antibody (R\&D systems).

\section{Immunofluorescence staining and confocal imaging}

Murine erythroid leukemia (MEL) cell line was transduced with MSCV-sPrdm16ires-mCherry vector to achieve $100 \%$ transduction efficiency. Cells transduced with MSCV-ires-mCherry vector were used as control. 2x10 6 transduced MEL cells were plated into Retronectin (Takara) coated cover slides 48 hours after transduction for 24 hours. Next day MEL cells were washed with $1 \mathrm{ml}$ warm DPBS twice gently and fixed with 4\% paraformaldehyde at room temperature (RT) for 1 hour. Cells were gently washed with DPBS twice before permeabilized with $0.1 \%$ triton 100 in DPBS for 15 mins at RT. After permeabilization cells were treated with Image-it TX signal enhancer (Invitrogen) for 30 mins at RT. Cells were stained with $10 \mathrm{ug} / \mathrm{ml}$ sheep anti-mouse PRDM16 polyclonal antibody (R\&D systems) in DPBS containing 10\% normal donkey serum (Sigma) at $4{ }^{\circ} \mathrm{C}$ overnight. Cells were washed by ice cold DPBS for 5 mins for three times next day before incubation with donkey-anti-sheep Alexa488 at 1:100 dilution in DPBS with 10\% normal donkey serum at RT for 1hour. Cells were rinsed with DPBS 3 times for 5mins each and stained with DAPI for 10 mins at RT and further rinsed with DPBS and were mounted for confocal image capture with Zeiss LSM 510 confocal microscope.

\section{Southern blot}

NIH-3T3 cells were transduced with CL20-MSCV-sPrdm16-ires-mCherry vector to achieve $\sim 60 \%$ transduction efficiency. Mock or CL20-MSCV-ires-mCherry control vector transduced cell were used as control. Genomic DNA was extracted with 
ArchivePure DNA Cell/Tissue kit (5 PRIME). 10ug genomic DNA was digested with 40 unites of DraI (NEB) at 344bp upstream of rev response element (RRE) and 102bp downstream of mCherry element to release $7.18 \mathrm{~kb}$ fragment. Genomic DNA migrated in $0.8 \%$ agarose gel at $20 \mathrm{~V}$ overnight, was transferred to Nytran SPC membrane (Fisher Scientific) and was hybridized dCTP-P32 labeled probe fragment complementary to RRE at $42^{\circ} \mathrm{C}$ overnight. Blot was washed and image was scanned next day.

\section{Results}

\section{Endogenous Prdm16 expression in LT-HSC and ST-HSCs}

The endogenous $\operatorname{Prdm} 16$ expression pattern consists of relatively high expression in CD34 ${ }^{-/ \mathrm{low}} \mathrm{Sca}^{+} \mathrm{c}-\mathrm{kit}^{+} \mathrm{Lin}^{-}$long term hematopoietic stem cells (LT-HSCs) and to a lesser extent in $\mathrm{CD}_{3} 4^{\text {high }} \mathrm{Scal}^{+}{ }^{+}$-kit ${ }^{+} \mathrm{Lin}^{-}$short term HSC cells (ST-HSCs) (Figure 3-3).

Endogenous expression levels were about 120 fold higher in LT-HSCs than in total bone marrow cells. Prdm 16 mRNA was down-regulated in myeloid and lymphoid progenitors at various stages of development as well as in mature blood cell lineages (Figure 3-3). These results showed that Prdm 16 expression is largely restricted to LT-HSCs where Prdm16 is required for HSC maintenance and function ${ }^{215,216}$.

\section{Overexpression of HOXA9 and HOXA10 led to growth advantage and enhanced colony forming capacity in vitro}

To exam whether overexpression of HOXA9 or HOXA10 was able to expand hematopoietic progenitors and enhance their self-renewal capacity in vitro, we transduced 5-FU treated bone marrow cells with retroviral vector containing either HOXA9 or HOXA10 cDNA. Cells overexpressing HOXB4 were included as positive control and control GFP transduced cells as negative control. During 15 days' culture HOXB4, HOXA9 and HOXA10 expressing cells showed significant grow advantage over control transduced cells. GFP or YFP percentage of HOXB4, HOXA9 or HOXA10 increased from about $10 \%$ to $85.87 \%, 93.83 \%$ and $96.47 \%$, respectively (Figure 3-4A). Colony forming capacity was also enhanced in HOXB4, HOXA9 and HOXA10 expressing cells (Figure 3-4B).

\section{Prdm16 is not expressed in cultured LSKs}

Endogenous Prdm 16 was shown to be highly expressed in WT murine LT-HSC and ST-HSC in previous experiment. We also assessed Prdm 16 transcript level in HOXB4, HOXA9 and HOX10 transduced LSK cells at day 4, 8 and 13 days post transduction in vitro. Compared with WT LSK cells, there wasn't detectable Prdm 16 transcript in GFP control transduced LSK cells in vitro and overexpression of HOXB4, 


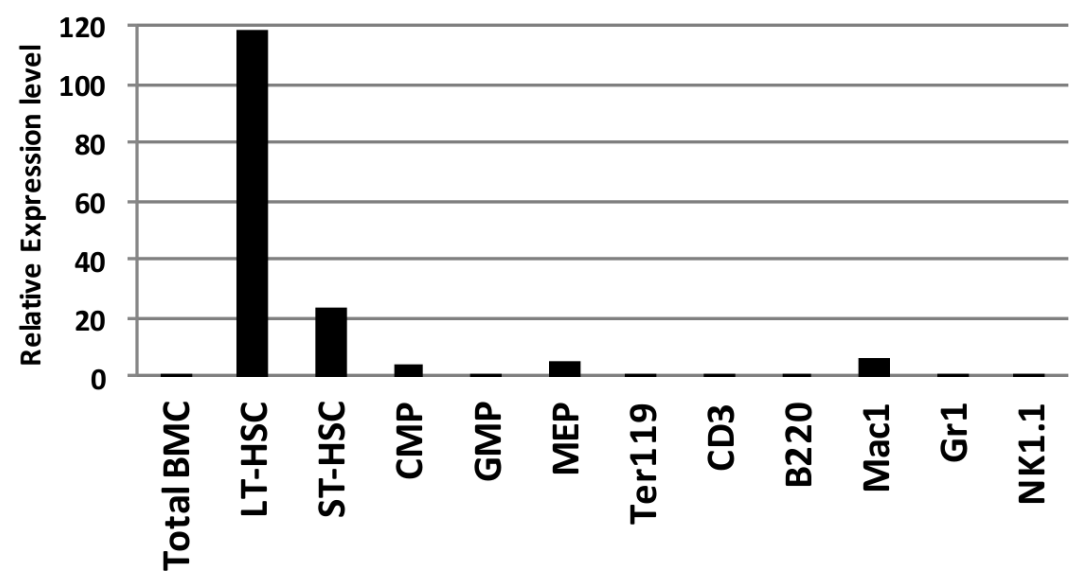

Figure 3-3. Quantitative real-time PCR of endogenous Prdm16 mRNA levels in various sorted progenitor and stem cell populations.

Endogenous Prdm16 was highly expressed in LT-HSCs and maintained relative high expression level in ST-HSCs. No or low level of Prdm16 transcript was detected in downstream progenitors and mature cells in bone marrow. Gapdh mRNA was used as a loading control and the level of Prdm16 mRNA expression in whole bone marrow was set at a relative value of 1.0. CMP: common myeloid progenitor; GMP: granulocytemonocyte progenitor; MEP: magakarocyte-erythroid progenitor. 
A.

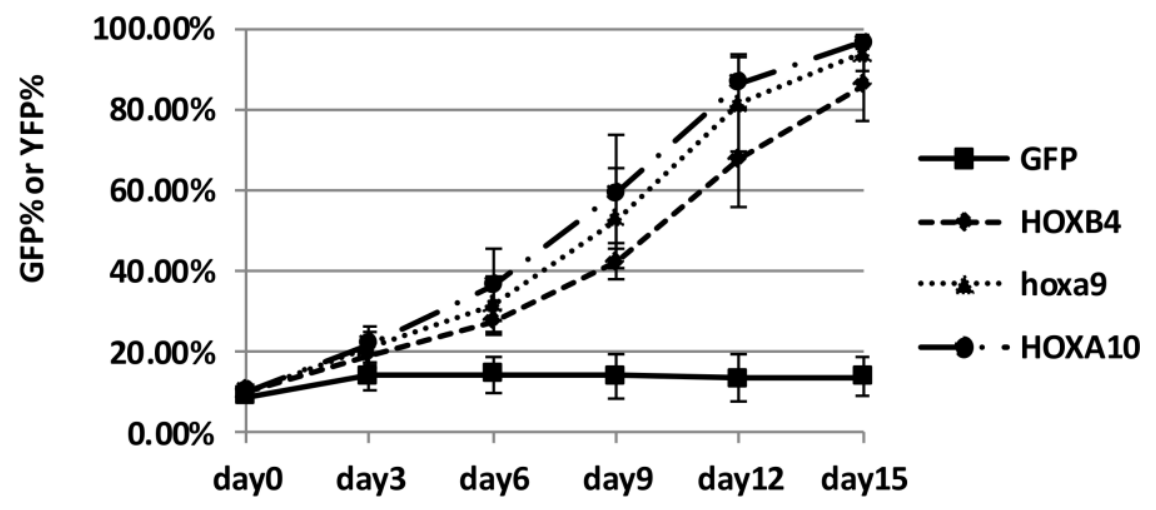

B.

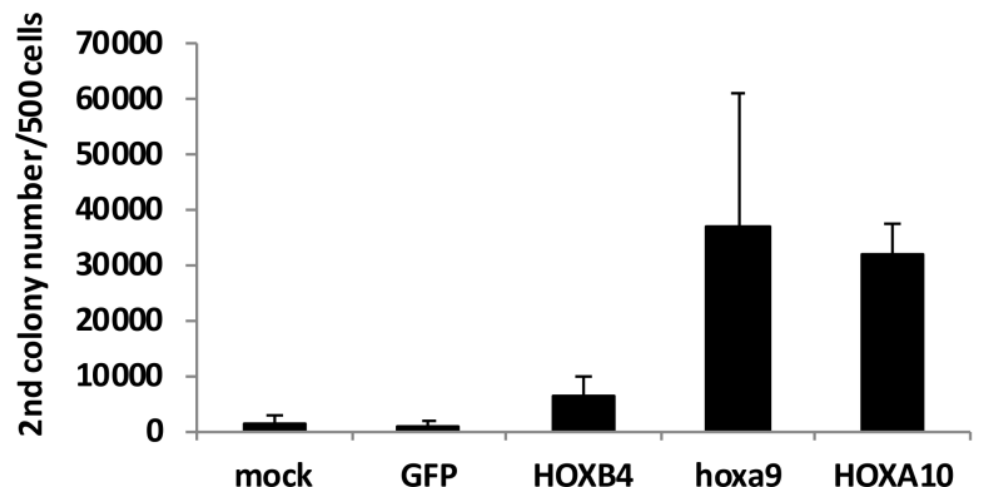

Figure 3-4. Growth advantage and enhanced myeloid progenitor self-renewal observed in HOXB4, HOXA9 and HOXA10 transduced cells in vitro.

(A) HOXB4, HOXA9 and HOXA10 demonstrated significant growth advantage over GFP control transduced cells during 15 days' culture. (B) HOXB4, HOXA9 and HOXA10 generated significant more secondary colonies than untransduced or GFP transduced cells. HOXA9 and HOXA10 demonstrated more robust colony forming capacity than HOXB4. 
HOXA9 and HOXA10 were not able to alter Prdm16 expression as they did in transplanted mice (Figure 3-5).

\section{Prdm16 downregulation is restricted to HOXB4-expressing HSCs in vivo}

HSC positive regulator, Prdm16, was downregulated in HOXB4-LSKs during the reconstitution, suggesting downregulation of $\operatorname{Prdm} 16$ can be a counter-regulatory signal which controlls HSC pool size or prevents leukemia in vivo. HOXA9 and HOXA10 are known oncogene, overexpression of which causes unlimited HSC expansion and leukemia in transplanted mice. We compared Prdm 16 expression level in HOXB4, HOXA9 or HOXA10 expressing CD150 ${ }^{+}$CD $48^{-}$LSKs (SLAM), CD $150^{+} \mathrm{CD}^{4} 8^{+} \mathrm{LSK}$ s and CD150 CD $48^{+}$LSKs (restricted progenitors) in transplanted mice at 6 weeks post transplantation. Compared with control GFP-SLAMs, Prdm 16 was 13.5 fold downregulated in HOXB4-SLAMs, but 5 fold upregulated in HOXA9-SLAM and 1.8 fold upregulated in HOXA10-SLAMs (Figure 3-6). Both HOXA9-SLAMs and HOXA10-SLAMs showed opposite trend of Prdm 16 expression compared to HOXB4SLAMs (Figure 3-6), indicating HOXB4 specifically downregulates Prdm16 to limit HSC pool size or to prevent leukemia. At meanwhile Prdm 16 expression level was not detachable in $\mathrm{CD} 150^{+} \mathrm{CD} 48^{+} \mathrm{LSKs}$ and $\mathrm{CD} 150^{-} \mathrm{CD} 48^{+} \mathrm{LSKs}$ in all groups (Figure 3-6), suggesting HOXB4 specific downregulation of Prdm16's was restricted in HSC compartment but not in downstream differentiated compartments.

\section{Lentiviral sPrdm16 construct and sPRDM16 protein expression}

To overexpress HOXB4 and sPRDM16 in mouse bone marrow cells, we generated lentiviral vector containing either HOXB4 with a GFP reporter gene driven by Efl $\alpha$ promoter or sPrdm16 with a linked mCherry gene downstream of MSCV promoter (Figure 3-7A). Western blot of vector transduced NIH-3T3 cells confirmed robust expression of HOXB4 and sPRDM16 protein (Figure 3-7B) and sPRDM16 protein was localized in nucleus when overexpressed in MEL cell line (Figure 3-7C).

\section{Low transduction efficiency of sPrdm16 overexpression vector}

To generate HOXB4 and sPRDM16 co-expressing cells, Lin ${ }^{-}$cells were transduced with HOXB4 and sPrdm16 vectors concurrently for 4 days (Figure 3-8A). Transduction efficiency of the bone marrow cells was evaluated 5 days post last transduction. We observed low proportion of cells expressing sPRDM16-mCherry protein (Figure 3-8B).

We followed sPrdm16-mCherry marking of transduced WT and p53 null cells in culture for up to 20 days post transduction. Rapid depletion of sPrdm16-mCherry positive WT and $p 53$ null cells was detected as early as 4 days post transduction (Figure 3-9A). Depletion of sPRDM16 expressing cells could be due to cellular toxicity caused 


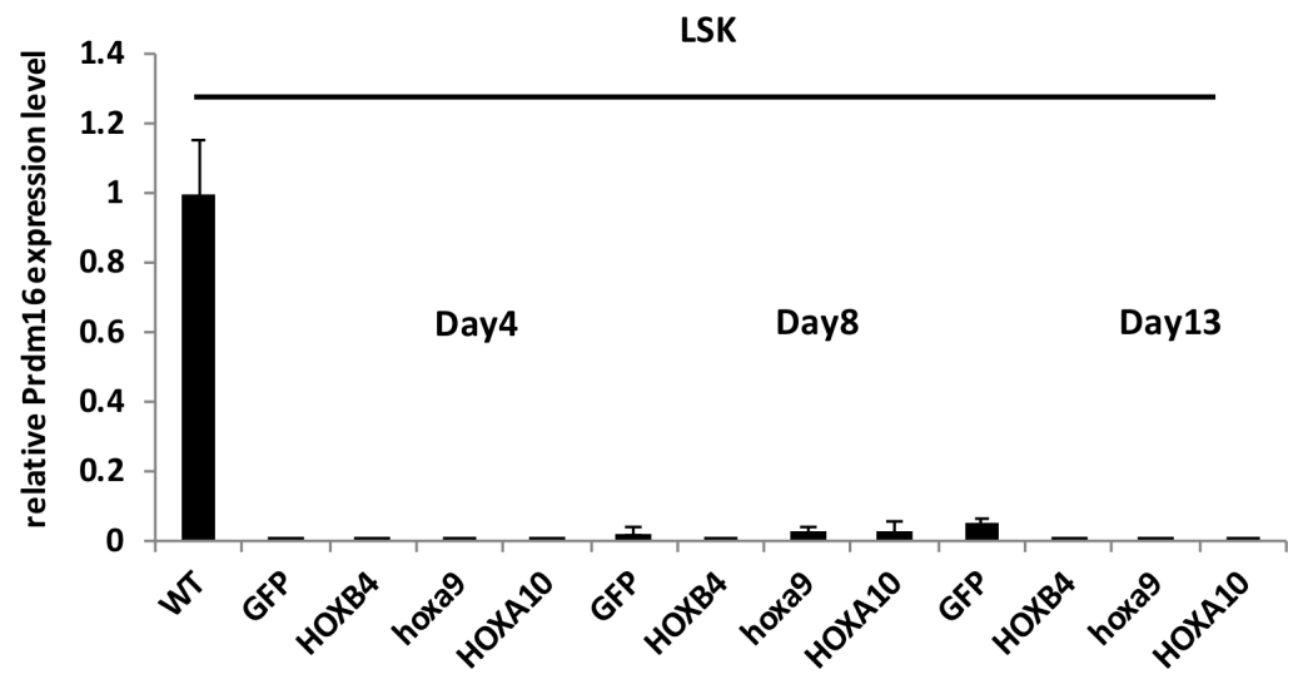

Figure 3-5. Relative Prdm16 expression in HOXB4, HOXA9 and HOXA10 transduced LSKs in vitro.

Prdm 16 transcript was measured in GFP, HOXB4, HOXA9 or HOXA10 transduced LSKs 4 days, 8 days and 13 days after transduction. Prdm 16 transcript was not detected in cultured LKSs in vitro compared with wild type LSKs. Gapdh mRNA was used as an endogenous control and the level of Prdm16 mRNA expression wild type LSKs was set at a relative value of 1.0 . 


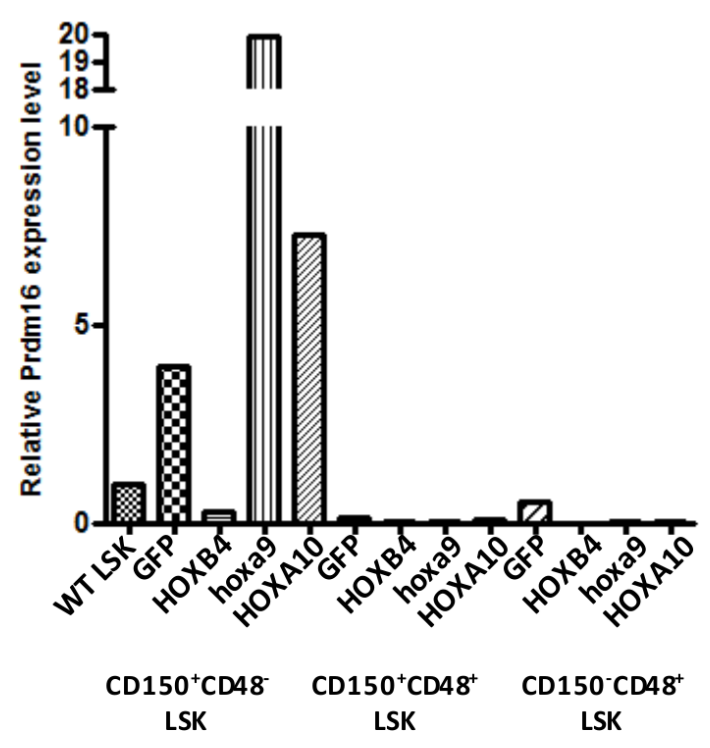

Figure 3-6. Downregluation of Prdm16 is restricted in HOXB4 expressing HSCs in vivo.

Prdm16 expression in HOXB4-SLAM was downregulated while upregulated in HOXA9SLAM and HOXA10-SLAM at 6 weeks post transplantation. Expression of Prdm 16 was not expressed in $\mathrm{CD} 150^{+} \mathrm{CD} 48^{+} \mathrm{LSKs}$ and $\mathrm{CD} 150^{-} \mathrm{CD} 48^{+} \mathrm{LSKs}$ in all groups. Gapdh mRNA was used as an endogenous control and the level of Prdm 16 mRNA expression wild type LSKs was set at a relative value of 1.0 . 
A.

CL20i4r-EF1 $\alpha$-GFP

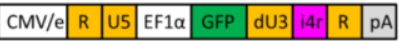

CL2014r-EF1 $\alpha$-HOXB4P2AGFP

\begin{tabular}{|l|l|l|l|l|l|l|l|l|l|l|}
\hline CMV/e & R & U5 & EF1 $\alpha$ & HOXB4 & P2A & GFP & dU3 & i4r & R & PA \\
\hline
\end{tabular}

CL20-MSCV-ires-mCherry

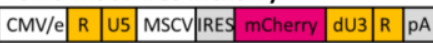

CL20-MSCV-sPRDM16-ires-mCherry

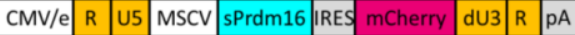

B.

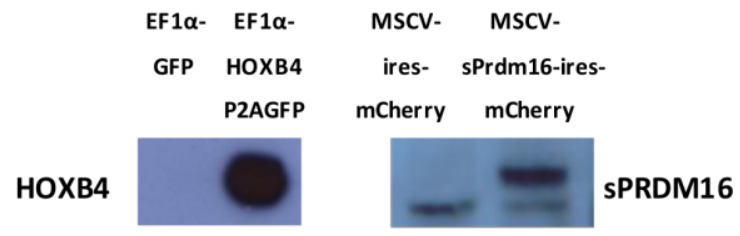

C.

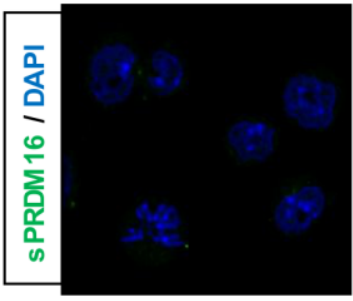

Control

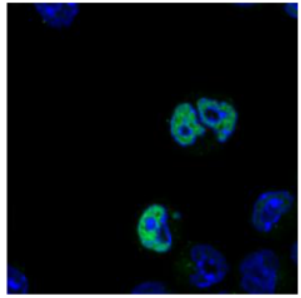

sPrdm16

Figure 3-7. Lentiviral vectors engineered to overexpress sPrdm16 and HOXB4. (A) Schematic representation of lentiviral vectors to overexpress HOXB4 and sPrdm16. The promoters and fluorescent reporter genes used are indicated. The $400 \mathrm{bp}$ chromatin insulator (i4r) was used as indicated to protect from position effects. (B) Western blot analysis of HOXB4 and sPrdm16 expression in NIH-3T3 cells transduced with the indicated vectors. Note the lower background band seen on the sPrmd 16 blot is also present with the "empty" vector control. (C) Immunofluorescence staining of sPrdm16 protein in MEL cell line.sPrdm16 protein was expressed in the nucleus. Green: sPrdm16; Blue: Dapi 
A.

\section{Concurrent Transduction}

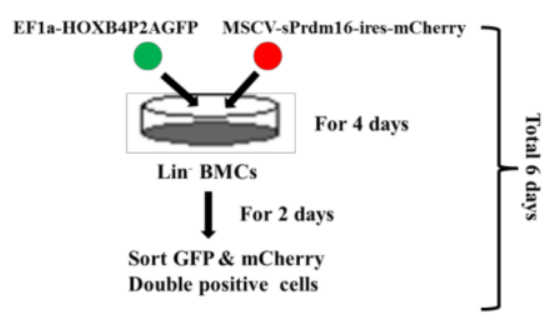

B.
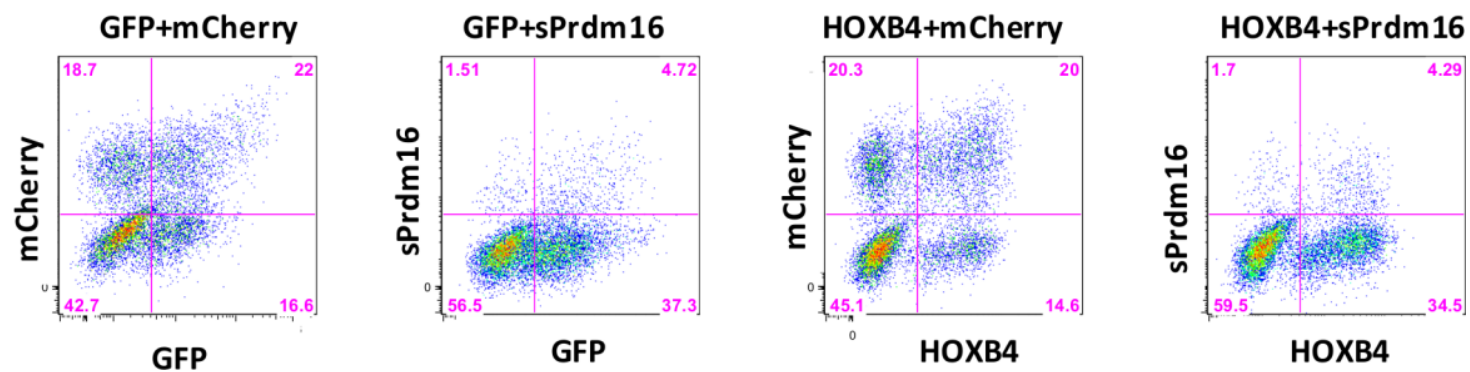

Figure 3-8. Low transduction of sPrdm16 vector in murine bone marrow cells. (A) Concurrent transduction includes 4 days transduction with two vectors simultaneously on Lin ${ }^{-}$bone marrow cells followed by FACS-based sorting 2 days after transduction. (B) Low co-transduction efficiency of sPrdm16 vector with GFP control vector or HOXB4 vector on BM cells evaluated 5 days post transduction. 
A.

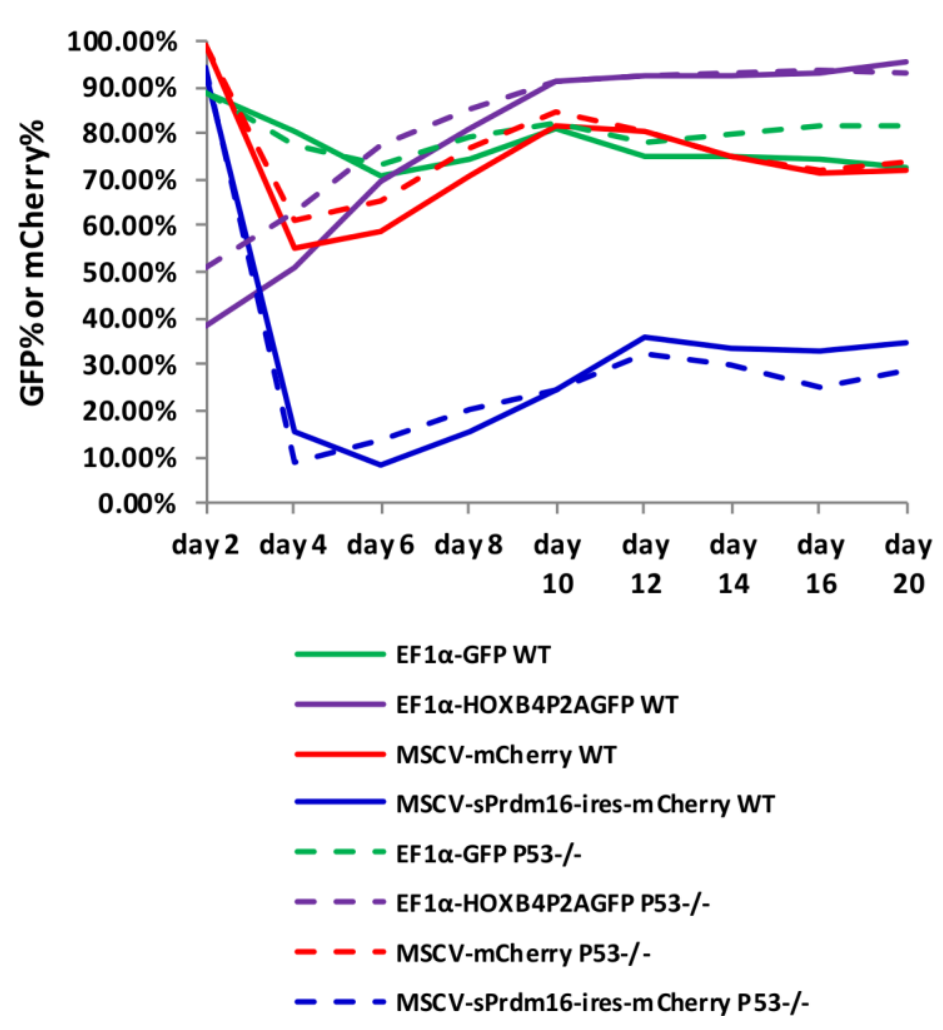

B.
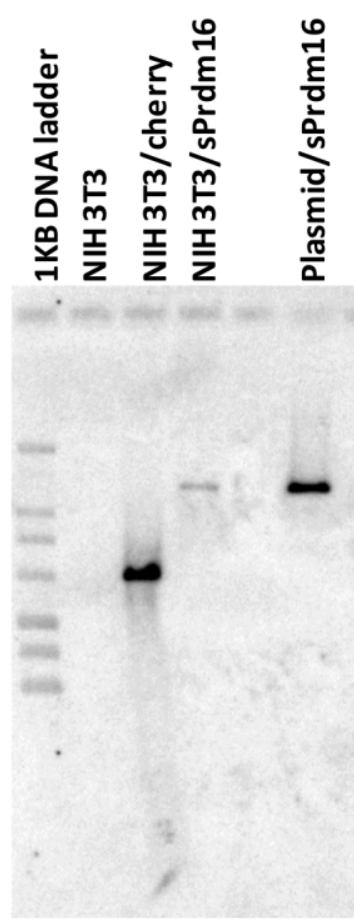

Figure 3-9. Depletion of sPRDMdfdf16 expressing cells was not due to vector rearrangement.

(A) GFP or mCherry marking of bone marrow cells in culture starting from 2 days post transduction. Experiment was done on either WT or P53 null mice. (B) Southern blot of NIH-3T3 cells transduced with CL20-MSCV-sPrdm16-ires-mCherry. CL20-MSCVsPrdm16-ires-mCherry plasmid was used as positive control. 
by unphysiologically high sPRDM16 protein level or vector instability during vector genome integration. So we tested sPrdm 16 vector rearrangement in transduced NIH-3T3 cells by southern blot. Compared with plasmid control, no rearrangement and deletion occurred between RRE and mCherry fragment in sPrdm16-mcherry transduced sample (Figure 3-9B), suggesting reduced sPrdm16-mcherry marking in bone marrow cells was not due to vector rearrangement.

\section{Increased colony forming capacity of HOXB4 and sPRDM16 coexpressing cells}

To test whether sPRDM16 and HOXB4 cooperate to enhanced hematopoietic progenitor cell self-renewal in vitro. Single and double transduced cells were sorted by flow sorting and analyzed for myeloid progenitor self-renewal using a serial replating assay. Cells transduced with the HOXB4 vector alone formed about 160 secondary colonies per $1 \times 10^{4}$ cells plated while sPrdm16-transduced cells formed about 70 secondary colonies (Figure 3-10). The HOXB4 and sPRDM16 double positive group formed over 300 secondary colonies, significantly more than the HOXB4 only group $(p<0.01)$ (Figure 3-10). This result demonstrates that HOXB4 and sPRDM16 synergize to enhance hematopoietic progenitor cell self-renewal.

\section{Enforced co-expression of sPRDM16 and HOXB4 causes preleukemia in mice}

We also transplanted co-transduced bone marrow cells into lethally irradiated recipient mice. Reconstitution with cells expressing the sPrdm 16 vector was generally very low, presumably reflecting the low titer of this vector or direct toxic effects of enforced sPrdm 16 expression. However, a large number of HOXB4 and sPrdm 16 double positive cells were detected in the peripheral blood in 2 of 12 recipients at 12 weeks after transplantation. In these two cases (\#890 and \#894), expansion of HOXB4 and sPrdm16 double positive blood cells occurred as early as 6 weeks after transplantation and further increased over time. When mouse \#894 was euthanized at 12 weeks post transplantation, $52.8 \%$ of peripheral blood cells and $97.1 \%$ of bone marrow cells co-expressed the HOXB4 and sPrdm 16 as determined by flow cytometry (Figure 3-11A). These double positive cells showed the Gr- $1^{+}$, Mac- $1^{+}$myeloid phenotype.

Bone marrow cells from mice $\# 890$ and $\# 894$ were used for secondary transplantation experiments. Secondary recipients from case \#894 did not engraft with donor cells. In contrast, secondary recipients generated from \#890 (\#10037, \#10038 and \#10040) were all repopulated with HOXB4 and sPrdm16 co-expressing cells in peripheral blood and bone marrow. At 12 and 16 weeks after transplant, pathological examination revealed prominent myeloid dysplasia with increased myeloid blasts in the bone marrow; consistent with myelodysplasia and pre-leukemia (Figure 3-11B). Taken together, this experiment directly shows that HOXB4 and sPrdm 16 overexpression collaborate to promote preleukemia in a fraction of secondary transplant recipients. 


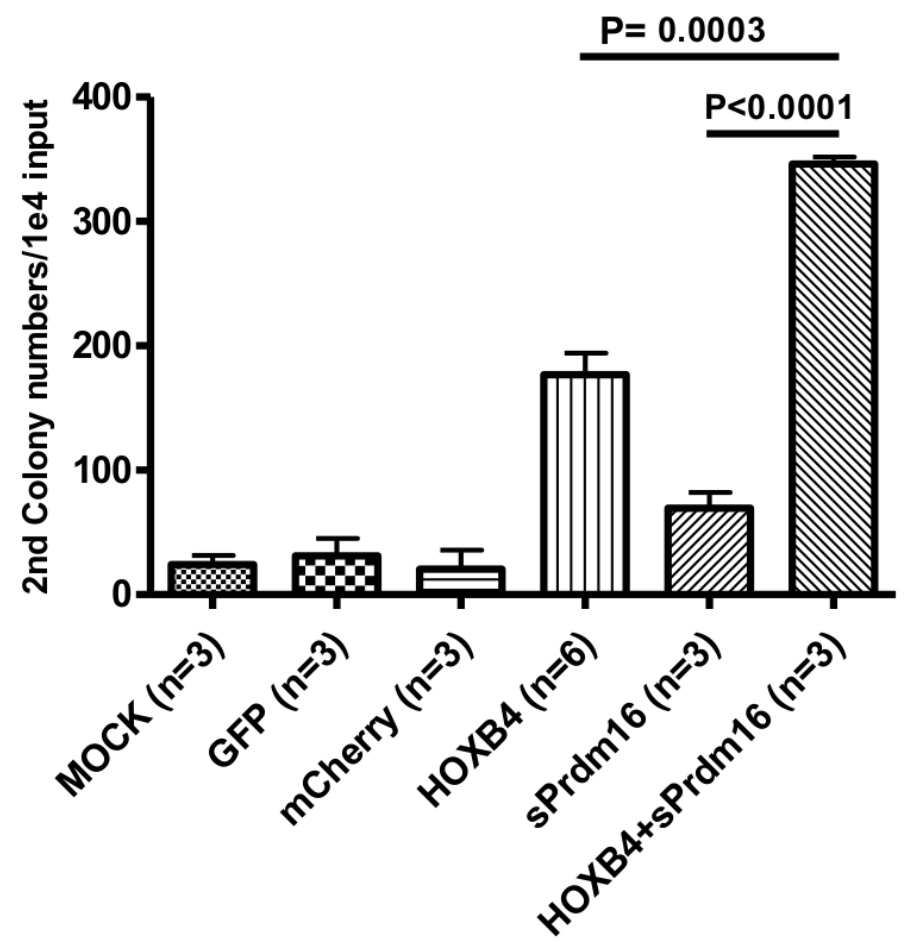

Figure 3-10. Secondary colony numbers by myeloid CFU-C assay on sorted transduced cells as a measure of self-renewal capacity.

Cells were transduced with control vectors or mock transduced, or with single HOXB4 or sPrdm 16 vectors, or co-transduced with both HOXB4 and sPrdm16 vectors as indicated. After a primary CFU-C assay, cells were replated and colonies scored based on 10,000 cell inputs. Error bars show the standard deviation for multiple experiments and statistical comparisons are indicated above the histograms. 
A.
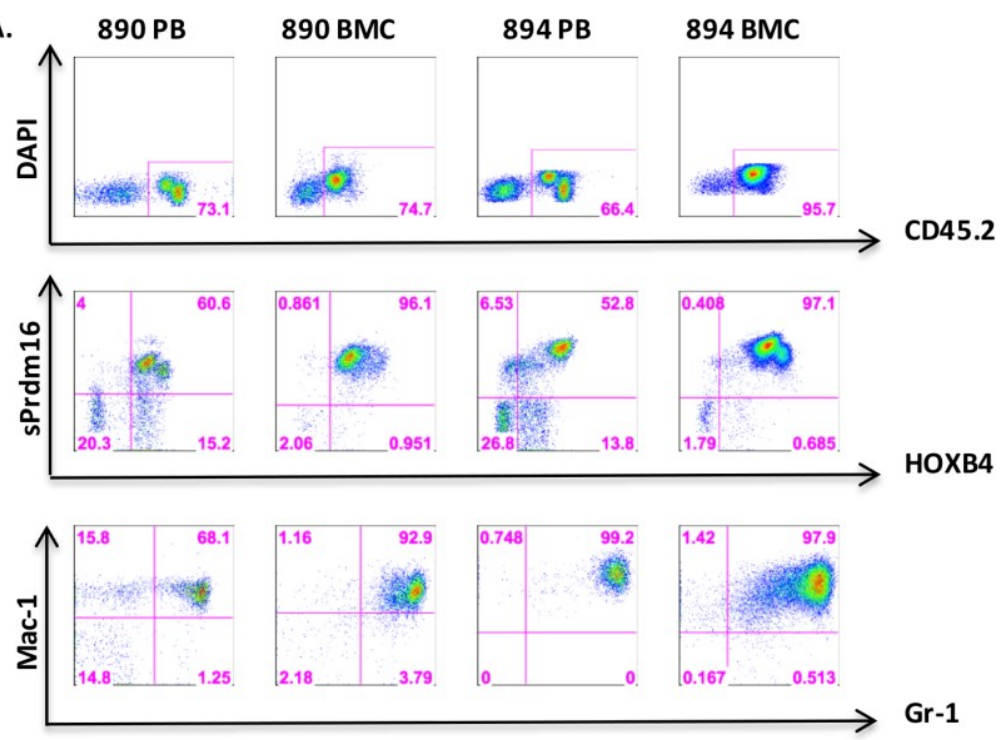

B.
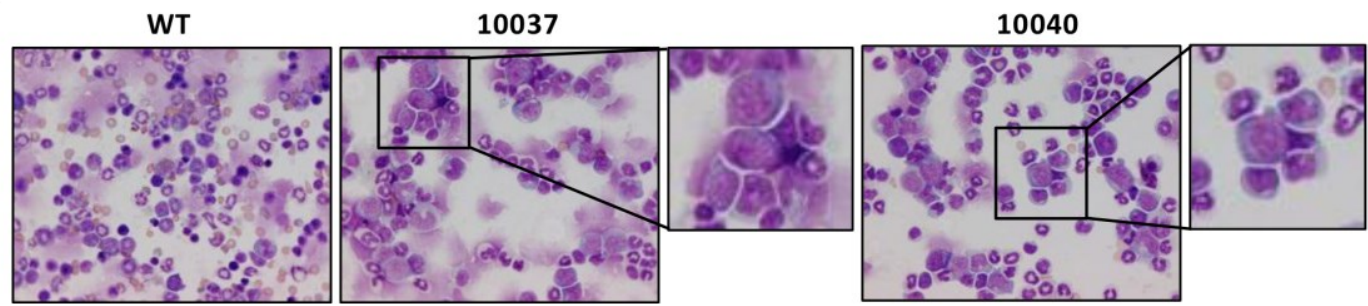

Figure 3-11. Enforced co-expression of HOXB4 and sPrdm16 enhances HPC selfrenewal in vitro and induces myeloid pre-leukemic transformation in transplanted mice.

(A) Flow cytometry analyses of HOXB4 and sPrdm16 double positive cells in peripheral blood (PB) and bone marrow cells (BMC) of two primary recipients, \# 890 and \# 894. The top row shows staining for the donor background CD45.1, the middle row shows expression of the sPrdm 16 vector marker (mCherry) and the HOXB4 vector marker (GFP), and the last row shows expression of the Mac-1 and Gr-1 myeloid markers. Note the expansion of double positive myeloid cells in each of these cases. (B) Bone marrow cytospin photomicrographs of secondary recipients (\#10037 and \#10040) derived by transplantation from donor \#890. Insets show higher magnification of the myeloid blasts seen in these cases, which comprised $30-50 \%$ of the cells on these slides. 


\section{Discussion}

Our gene expression array data from HOXB4 expressing LSK cells demonstrated marked downregulation of Prdm16. By reviewing previous literature and independent screening of HOXB4 transplanted mice, we hypothesized that downregulation of Prdm 16 is the specific mechanism how HOXB4 prevent leukemia in transplanted mice. Here we talk about 'HOXB4-specific mechanism' because HOXA9 and HOXA10, oncogenic HOX factors, upregulated Prdm 16 expression up to about 50 fold in HSCs compared with HOXB4. Differential regulation of Prdm16 by HOXB4, HOXA9 and HOXA10 was not seen in culture, as $\operatorname{Prdm} 16$ expression was no longer detectable in vitro, suggesting extrinsic factors present in bone marrow niche are required to maintain $\operatorname{Prdm} 16$ expression in HSCs. However, there are no available methods for phenotypically identifying HSCs in these long term cultures. While we gated on LSK cells after various times in culture, published data ${ }^{262,263}$ suggests that these markers, or any other for that matter, are not adequate for prospective identification of HSC-enriched populations in vitro.

To further validate that downregulation of $\operatorname{Prdm} 16$ is responsible for preventing leukemic transformation in HOXB4 transplanted mice, we can co-overexpressed HOXB4 and sPRDM16 in murine primitive bone marrow cells to induce leukemia. At meanwhile knockdown Prdm 16 in HOXA9 or HOXA10 transplanted mice prior or after leukemia onset will be anther experiment to test sustained expression of Prdm 16 as a major player in leukemogenesis. But this part of experiment will not be included in this project.

We designed lentiviral vectors to overexpress HOXB4 and SPRDM16 in murine hematopoietic primitive cells. HOXB4 and sPRDM16 protein expression was confirmed in transduced NIH-3T3 cells. However, we noticed very low (less than 5\%) cotransduction efficiency of sPrdm 16 with control GFP vector and HOXB4 vector. This may reflect the low titer of the sPrdm16 vector. But it also can be due to vector rearrangement during integration. Southern blot done on sPrdm16 transduced NIH-3T3 cells detected intact transgene fragment, excluding the possibility of vector rearrangement. As mentioned above, Prdm16 is selectively expressed at high level in HSCs. sPRDM16 expression level of by overexpression vector probably was not favorable in transduced progenitor cells in culture. This explains why Prdm 16 was not detectable in culture cells and marked decreased proportion of Prdm16 transduced cells in vitro.

Though low transduction of Prdm16 vector makes it difficult to overexpress Prdm 16 using constitutive overexpressing vector, we were able to purify 500 double transduced cells for colony forming assay to test both factors' effect on the self-renewal capacity of myeloid progenitors. We injected bulk transduced cells for transplantation assay to screen leukemia in mice due to inadequate mount of double cells for transplantation. Since we hypothesize that downregulation of Prdm 16 prevents leukemogenesis inHOXB4 transplanted mice, we expect leukemia derived from only HOXB4 and sPrdm16 double positive cells but not any other populations. We detected two preleukemia cases (myeloid dysplasia) out of 12 primary recipients at 28 weeks and 
32 weeks following primary transplantation. Long latency of leukemogenesis reflects the low number of leukemia initiating cells due to the poor co-transduction efficiency or the requirement of additional mutations for the leukemic transformation. The mechanisms by which sPrdm 16 cooperates with HOXB4 are yet undefined. Expression of sPRDM16 blocks granulocyte-colony stimulating factor (G-CSF)-induced myeloid differentiation ${ }^{198,238,239}$ and over-expression of sPRDM16 in mice causes a myeloproliferative disorder and leukemia in $p 53$-null bone marrow cells. ${ }^{248}$ Therefore, in the context of HOXB4-induced HSC expansion, downregulation of Prdm 16 functions to induce hematopoietic differentiation, thus preventing leukemia in vivo.

To address the low transduction efficiency of sPrdm16 lentiviral vector, TetOn inducible vector which is able to activate transgene expression upon doxycycline induction at dose-dependent manner can be a great replacement of constitutive overexpression vector. We decide to establish TetOn-sPrdm16 transgenic mouse to further study the role of Prdm 16 in HSC expansion and leukemogenesis. The advantage of transgenic mouse over the vector transduction is the stable and predictable transgene integration rather than random vector integration between one transduction and another. We plan to transplant $s$ Prdm 16 transgenic bone marrow cells into wild type recipients to avoid side effect of sPRDM16 overexpression in other organs. 


\section{CHAPTER 4. DOWNREGULATION OF PRDM16 IS REQUIRED FOR HOXB4-MEDIATED HSC EXPANSION}

\section{Introduction}

In previous chapter downregulation of $\operatorname{Prdm} 16$ was shown as HOXB4-specific mechanism to prevent leukemia in transplanted mice. Besides avoiding leukemic transformation, well-regulated HSC pool size in HOXB4 recipient mice is another feather of HOXB4-mediated HSC expansion, ${ }^{141,181,182}$ mechanism of which is not clear. Induction of differentiation, apoptosis or quiescence in HSCs post dramatic proliferation can contribute to maintain HSC pool size at physiological level. Prdm 16 is selective expressed in LT-HSCs. ${ }^{215,216}$ Sustained expression of Prdm16 HSCs can maintain HSC's quiescence, and loss of Prdm16 may drive HSC into cell cycle, thus allowing proliferation and expansion. Given the fact that loss of Prdm 16 indeed increased the frequency of HSCs in S/G2/M phase of the cell cycle, ${ }^{215,216}$ and Prdm16 was markedly repressed by HOXB4 in vivo, we hypothesize that HOXB4 downregulates Prdm16 to allow HSC to undergo proliferation. To test the hypothesis we will assess CRU frequency in HOXB4 transplanted mice present or absent of $s$ Prdm 16 overexpression. Considering the poor transduction of sPrdm16 lentiviral vector, we will generate TetOn-sPrdm16 transgenic mice, bone marrow of which will be used for experiments. We expect sPRDM16 protein expression upon doxycycline administration in transgenic bone marrow cells and lower CRU frequency in HOXB4 and sPRDM16 coexpressing cells than HOXB4 only expressing cells.

\section{Materials and Methods}

\section{Vector cloning}

To generate TetOminiCMV-sPrdm16-P2A-GFP-rGBpA vector, sPrdm16 cDNA was released from MSCV-sPrdm16-ires-mCherry vector using EcoRI and replaced in front of HOXB4 cDNA in EF1a-HOXB4-P2A-GFP vector to generated EF1a-sPrdm16HOXB4-P2A-GFP vector. Synthesize partial sPrdm16-P2A fragment was generated by Biomatik with DraIII in 5'end and BsmBI in the 3' end, which was replaced with partial sPrdm16-HOXB4-P2A fragment in EF1a-sPrdm16-HOXB4-P2A-GFP vector. In the end Efla-sPrdm16-P2A-GFP vector was established. Fragment sPrdm16-P2A-GFP was further released from backbone with EcoRI and NotI and EcoRI site in the 5' end was blunted using Klenow. Lentiviral vector pHAGE2-TetOminiCMV-Oct4F2AK1f4-iresSox2E2AcMyc-W was offered by Charles Sherr in St. Jude Children's Research Hospital. Fragment Oct4F2AK1f4-ires-Sox2E2AcMyc was removed from backbone using NotI and ClaI and NotI site present in backbone was blunted by Klenow. sPrdm16P2A-GFP fragment was ligated into the pHAGE2-TetOminiCMV backbone to generate pHAGE2-TetOminiCMV-sPrdm16-P2A-GFP lentiviral vector. rGBpA fragment was amplified by PCR from Prdm16 knockdown vector and further digested by HindIII to 
release the exact rGBpA fragment. Previous generated pHAGE2-TetOminiCMVsPrdm16-P2A vector was digested with NotI and blunted by Klenow. HindIII digested rGBpA was also blunted by Klenow and replaced into pHAGE2-TetOminiCMVsPrdm 16-P2A after P2A fragment to generate pHAGE2-TetOminiCMV-sPrdm16-P2ArGBpA vector. pHAGE2-TetOminiCMV-sPrdm16-P2A-rGBpA circular vector was digested by XhoI and NotI to release linearized TetOminiCMV-sPrdm16-P2A-rGBpA for microinjection.

CMV-rtTA lentiviral vector was kindly provided by Dr. Charles Sherr in St. Jude Children's Research Hospital.

\section{Calcium phosphate transfection and vector concentration for lentiviral vector}

Calcium phosphate transfection and ultracentrifugation of TetOminiCMVsPrdm16-P2A-GFP lentiviral vector were carried out in the same way described in Chapter 3.

\section{SPRDM16 protein induction in vitro}

Prior to microinjection of linearized plasmid to mice zygotes, NIH3T3 cells were co-transduced with TetOminiCMV-sPrdm16-P2A-GFP and CMV-rtTA lentiviral vectors. 48 hours post transduction, NIH3T3 cells were treated with $0 \mathrm{ng} / \mathrm{ml}, 0.1 \mathrm{ng} / \mathrm{ml}, 1 \mathrm{ng} / \mathrm{ml}$, $10 \mathrm{ng} / \mathrm{ml}, 100 \mathrm{ng} / \mathrm{ml}$ and $1000 \mathrm{ng} / \mathrm{ml}$ of doxycycline for 48 hours and doxycycline was withdrew from medium. GFP fluorescence was evaluated by flow cytometery after 48 hours' induction and 5days' withdraw and protein expression was examined by western blot after 48 hours' induction.

\section{Mouse strain maintenance and breeding}

All mouse maintenance and breeding procedures were carried out according to protocols approved by the St. Jude Institutional Animal Care and Use committee. Transgenic founder mice were bred with B6.Cg-Gt(ROSA)26Sor $<\operatorname{tm} 1\left(\mathrm{rtTA}^{*} \mathrm{M} 2\right) \mathrm{Jae}>/ \mathrm{J}$ mice (Jackson Lab, stock number 006965) to generate N1 mice carrying both transgene and rtTA knockin gene and then further bred with B6.Cg$\mathrm{Gt}(\mathrm{ROSA}) 26 \mathrm{Sor}<\mathrm{tm} 1(\mathrm{rtTA} * \mathrm{M} 2) \mathrm{Jae}>/ \mathrm{J}$ mice to generate $\mathrm{rtTA}$ homozygote transgenices.

\section{Polymerase chain reaction (PCR) genotyping}

To confirm the presence of transgene in the genome of progenies, PCR to amplify the fragment between $s$ Prdm 16 cDNA and green fluorescence protein (GFP) was used for genotyping. Genomic DNA was extracted from tails and about 10ng of genomic DNA was subjected to PCR with HotMaster Mix (5 PRIME). PCR primer sequences were 
GCTGGAGGAAGAGGATGATG (forward) and GAACTCCAGCAGGACCATGT (reverse) and expected PCR product size was $892 \mathrm{bp}$. At meanwhile PCR to amplify endogenous Actin was carried out as control reaction.

To confirm presence of rtTA knockin sequence in the genome of progenies, PCR was carried out according to Jackson Lab's instruction. Mutant reverse primer oIMR8052-- GCG AAG AGT TTG TCC TCA ACC, WT reverse primer oIMR8546-GGA GCG GGA GAA ATG GAT ATG and common primer oIMR8545-- AAA GTC GCT CTG AGT TGT TAT were used for genotyping. Expected size of WT product is about $650 \mathrm{bps}$, size of rtTA heterozygote is $340 \mathrm{bp}$ and $650 \mathrm{bp}$ and rtTA homozygotes is $340 \mathrm{bp}$.

No template control and WT control were included for both PCR reactions.

\section{Doxycycline drinking water administration}

To induce transgene expression in vivo, transgenic mice or transplanted mice were subjected to $2 \mathrm{mg} / \mathrm{ml}$ doxycycline (Sigma) treatment in drinking water with $50 \mathrm{mg} / \mathrm{ml}$ sucrose (Sigma). Drinking water without doxycycline was used as control. Drinking water was filtered with Corning disposable sterile filter system (Corning) and changed every 3 days.

\section{Western blot}

PCR positive transgenic mice and control littermates were under $2 \mathrm{mg} / \mathrm{ml}$ doxycycline treatment for continuous 7days. GFP positive and negative BM cells were sorted. $1 \times 10^{6}$ sorted cells were washed with $1 \mathrm{ml}$ ice cold DPBS, resuspended with 50ul ice cold DPBS with 2x Halt Protease Inhibitor Cocktail with EDTA (Thermo Scientific Pierce) and 50ul Laemmli sample buffer (Bio-Rad) supplemented with 2Mercaptoethanol (Sigma) and boiled at $99{ }^{\circ} \mathrm{C}$ for 5 minuts before being placed on dry ice. 40ul cell lysate (from 4e5 cells) were load in 4\%-12\%SDS-PAGE gel (Invitrogen) for protein detection. sPRDM16 protein was probed with $0.1 \mathrm{ug} / \mathrm{ml}$ sheep anti-mouse PRDM16 polyclonal antibody (R\&D systems) followed by HRP-conjugated donkey antisheep secondary antibody (R\&D systems). GFP protein was probed with rabbit GFP antibody (Cell signaling) at 1:5000 dilution followed by HRP-conjugated goat anti-rabbit secondary antibody (Santa Cruz Biotechnology). GAPDH protein was probed with mouse anti-mouse GAPDH antibody (Millipore) at 1:50000 dilution followed by HRPconjugated goat anti-mouse $\operatorname{IgG}_{1}$ antibody (Santa Cruz Biotechnology).

\section{Southern blot}

About 3-10ug tail genomic DNA was digested with sphI-HF in 40ul reaction at 37 ${ }^{\circ} \mathrm{C}$ for 8 hours to check transgene copy number or digested with DraI and PshAI to check 
transgene integrity. DNA gel electrophoresis, transfer and hybridization and image detection was carried as described in chapter 3 . The probe used for hybridization was complementary to GFP sequence.

\section{Fluorescence in situ hybridization (FISH)}

Bone marrow cells from transgenic mice were submitted to Cytogenetic Shared Resource of St. Jude Children's Research Hospital to determine integration localization of transgene by FISH. Purified TetOminCMV-sPrdm16-P2A-GFP-rGBpA plasmid DNA was labeled with a green-dUTP by nick translation and chromosome control clone was labeled with a red-dUTP. Labled probes were combined with sheared mouse DNA and hybridized to matophase chromosomes derived from the bone marrow cells. The chromosomes were stained with DAPI and analyzed.

\section{Fluorescence flow cytometry analysis}

To detect phonotypical HSC and MPP frequency in transplanted mice, bone marrow cells were stained with CD150-PE-Cy7, CD48-APC, Sca-1-Percp5.5, c-KitAPC-eF780, CD45.2-V500 and eF605 conjugated-lineage cocktail (BD Bioscience). SLAM (CD150 ${ }^{+} \mathrm{CD}^{-} 8^{-} \mathrm{Sca}-1^{+} \mathrm{c}-\mathrm{Kit}^{+}$Lineage $\left.{ }^{-}\right)$and MPP (Sca- $1^{+} \mathrm{c}-\mathrm{Kit}^{-}$Lineage $\left.{ }^{-}\right)$population were gated according to isotype and single color controls.

\section{Limiting dilution assay}

For limiting dilution assay to assess CRU frequency, BM cells of transgenic mice were harvested 5 days after 5-FU treatment, prestimulated as describe in chapter 2. After prestimulation $5 \times 10^{5}$ bone marrow cells were transduced with MSCV-HOXB4-iresmCherry vector overnight. Control vector MSCV-ires-mCherry vector was included as control. Next day after transduction $5 \times 10^{5}$ or $1 \times 10^{6}$ were injected into lethally irradiated B6.SJL-PtprcaPepcb/BoyJ mice (Jackson Lab). Recipients were under Baytril water treatment for 3 weeks followed by doxycycline $(2 \mathrm{mg} / \mathrm{ml})$ treatment. Drinking water without doxycycline was used as control. Bone marrow cells were harvested from primary recipients at 18 weeks post transplantation and $320,1600,8000,4 \times 10^{4}$ and $2 \times 10^{5}$ bone marrow cells were injected into letherally irradiated B6.SJL-PtprcaPepcb/BoyJ mice (Jackson Lab) together with 2x10 5 B6.SJL-PtprcaPepcb/BoyJ (Jackson Lab) bone marrow cells. Recipients' PB was analyzed 18 weeks post secondary transplantation. Mouse with more than 1\% mCherry marking in each lineage (Gr1 and Mac1, CD3, B220, Ter119) was considered as a positive recipient. CRU frequency was calculated using LCalc $^{\mathrm{TM}}$ software (Stemsoft Sotfware Inc. version 1.1). 


\section{Results}

\section{Vector construction}

We generated lentiviral vector to overexpress murine $s$ Prdm 16 driven by TetOminiCMV promoter followed by P2A-GFP (Figure 4-1A). For microinjection to generate TetOminiCMV-sPrdm16-P2A-GFP-rGBpA transgenic mice, rBGpA fragment was ligated into vector after GFP. Linearized TetOminiCMV-sPrdm16-P2A-GFPrGBpA DNA fragment (Figure 4-1A) was injected in fertilized zygotes at singe cell stage.

\section{Dose-dependent induction of sPRDM16 and GFP protein}

NIH-3T3 cells were co-transduced with TetOminiCMV-sPrdm16-P2A-GFP and CMV-rtTA vectors (Figure 4-1A) and further induced by $0-1000 \mathrm{ng} / \mathrm{ml}$ doxycycline for 48 hours in vitro. $0 \mathrm{ng} / \mathrm{ml}, 0.1 \mathrm{ng} / \mathrm{ml}$ and $1 \mathrm{ng} / \mathrm{ml}$ doxycycline did not induce significant GFP expression (baseline), 10ng/ml doxycycline led to mediate GFP induction while $100 \mathrm{ng} / \mathrm{ml}$ and $1000 \mathrm{ng} / \mathrm{ml}$ doxycycline achieved maximum induction of GFP expression (Figure 4-1B). GFP expression decreased to baseline 5 days after doxycycline was withdrew (Figure 4-1B). sPRDM16 and GFP protein was detected in 10ng/ml, 100ng/ml and $1000 \mathrm{ng} / \mathrm{ml}$ samples 48 hours after induction by western blot, which was consistent with GFP fluorescence signal by flow cytometry (Figure 4-1C). sPRDM16 and GFP protein expression showed nice linear correlation (Figure 4-1C). Both protein inductions were maximized at dose of $100 \mathrm{ng} / \mathrm{ml}$ without no further increase of protein expression at 1000ng/ml doxycycline (Figure 4-1C).

\section{Generation and validation of TetO-sPrdm16 transgenic mice}

To achieve inducible sPRDM16 protein expression in mouse bone marrow cells, we generated TetO-sPrdm16 transgenic mice. Transgenic founder mice carrying one allele of transgene was bred with B6.Cg-Gt(ROSA)26Sor $<\operatorname{tm} 1\left(\mathrm{rtTA}^{*} \mathrm{M} 2\right) \mathrm{Jae}>/ \mathrm{J}$ mice (Jackson Lab, stock number 006965) to generate N1 mice, which were able to expression sPRDM16 protein under doxycycline induction (Figure 4-2). N1progenies were further bred with $\mathrm{rtTA}^{*} \mathrm{M} 2$ mice to achieve two allele of rtTA gene in genome.

PCR amplifying the transgene region was carried out on mouse tail genomic DNA to check the presence or absence of transgene in mouse genome. PCR primers were designed to amplify between sPrdm 16 and GFP region to yield $892 \mathrm{bp}$ product as shown in lane 1, 5, 9, 11 and positive control (Figure 4-3A, B). Total 21 PCR positive transgenic founders were identified, 7 of which were c57BL/6J strain and others were FVB strain. PCR were carried out on N1 genomic DNA and 17 out of 21 founders had germline transmission to $\mathrm{N} 1$ and following progenies. PCR was carried out according to Jackson Lab's instruction to detect WT, heterozygotes and homozygotes of rtTA gene. 
A.

B.

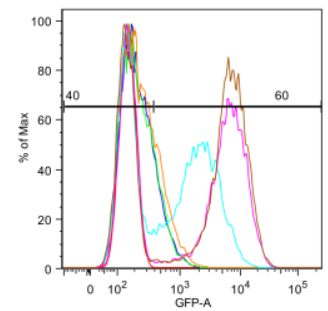

$$
\text { 48h post }
$$
Doxinduction

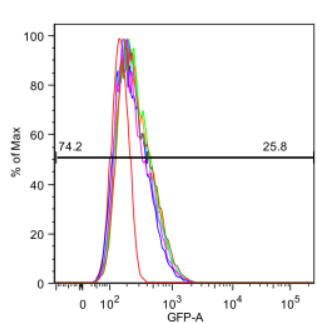

5 days post Dox withdraw

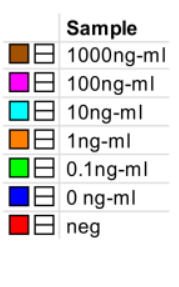

C.

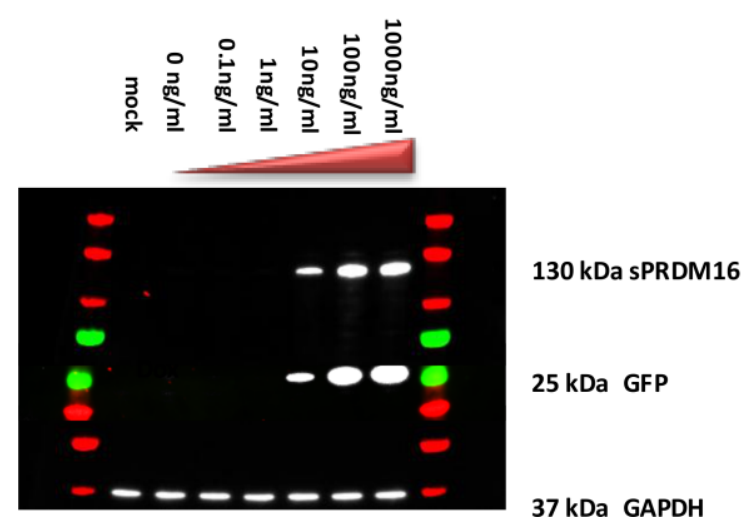

Figure 4-1. Dose-dependent induction of sPRDM16 and GFP protein with TetOn inducible system.

(A). Schematic diagram of TetOminiCMV-sPrdm16-P2A-GFP and CMV-rtTA lentiviral vectors and linearized TetOminiCMV-sPrdm16-P2A-GFP-rBGpA. (B) GFP fluorescence detected in transduced NIH-3T3 cells 48 hours post Doxcycline induction and decreased back to baseline 5 days after Doxycycline withdraw.(C): sPRDM16 and GFP protein was detected in induced NIH-3T3 cells at dose of 10, 100 and 1000ng/ml Doxycycline after 48 hours' induction. 


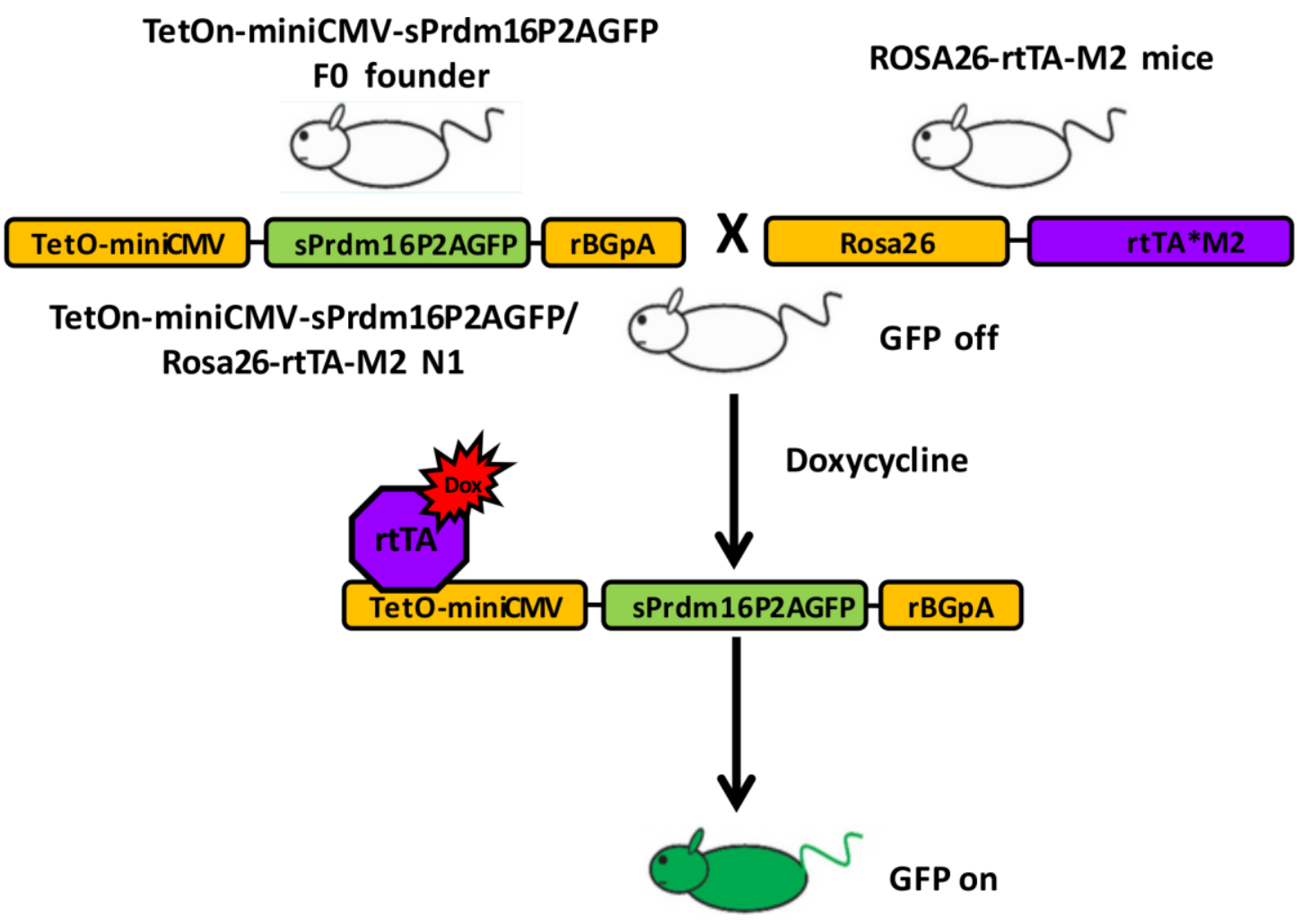

Figure 4-2. Schematic diagram of TetO-sPrdm16 transgenic mice generation.

Transgenic founder mice were Bred with B6.Cg-Gt(ROSA)26Sor $<\operatorname{tm} 1(\mathrm{rtTA} * \mathrm{M} 2) \mathrm{Jae}>/ \mathrm{J}$ mice to generate $\mathrm{N} 1$ mice carrying both transgene and rtTA knockin gene. 
A.

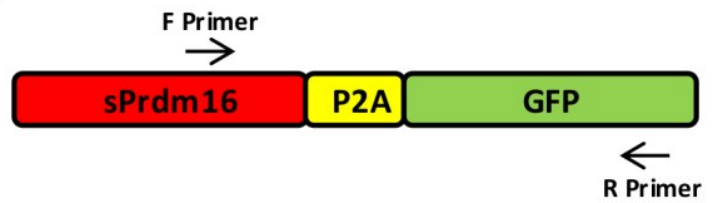

B.

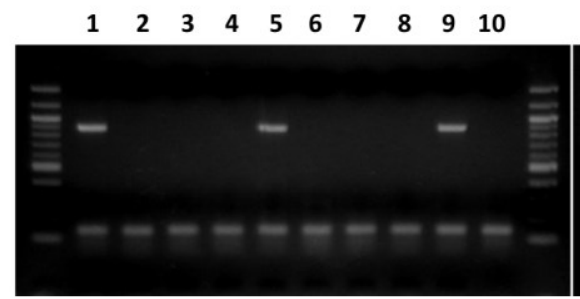

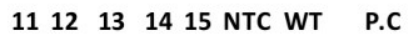

C.

$\begin{array}{llllllllll}1 & 2 & 3 & 4 & 5 & 6 & 7 & \text { NTC } & \text { WT rtTA } & \end{array}$

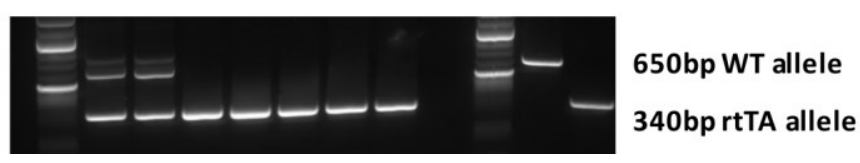

Figure 4-3. TetO-sPrdm16 transgenic mice PCR genotyping.

(A). Forward and reverse primers designed to amplify partial sPrdm16-P2A-GFP region to detect presence of transgene in mouse genome. (B). Example of sPrdm16 transgene PCR genotyping. Lane 1, 5, 9 and 11 are transgene positive compared with non-template control (NTC), wild type (WT) control and positive control (P.C). (C). Example of rtTA knockin gene PCR genotyping. Compared with NTC, WT and rtTA ${ }^{+/+}$control, lane 1 and 2 are $\operatorname{rtTA}^{+/-}$and lane 3-7 are $\mathrm{rtTA}^{+/+}$. 
Expected size of WT product is about $650 \mathrm{bps}$ (WT control lane), size of rtTA heterozygote is 340bp and 650bp (lane 1 and 2) and rtTA homozygotes is 340bp (lane 37) (Figure 4-3C). $2 \mathrm{mg} / \mathrm{ml}$ Doxycycline in drinking water was given to three litters of N1 mice with germline transmission for 7 days to detect GFP fluorescence in nucleated cells from PB.by flow cytometry. 9 out of 17 founders' N1 progenies demonstrated inducible and reversibl GFP expression in PB with different level of GFP induction efficiency

(Figure 4-4A, B).

To check transgene copy number and integrity in mouse genome, southern blot was carried out on founders $(8012,8005,8080,8018$ and 8017) and progenies with the best GFP induction. Southern blot of founder 8012, 8080, 8018 and 8017 showed single copy of transgene with one integration in mouse genome while founder 8005 had more than one integration and more than one copy of transgene (Figure 4-5A). We also detected rearranged or deletion of transgene from 8005 genome (Figure 4-5A). To segregate rearranged or deleted transgene we further bred 8005 with $\mathrm{B} 6 . \mathrm{Cg}$ $\mathrm{Gt}(\mathrm{ROSA}) 26 \mathrm{Sor}<\mathrm{tm} 1(\mathrm{rtTA} * \mathrm{M} 2) \mathrm{Jae}>/ \mathrm{J}$ mice and carried out southern blot on four N1 progenies $(8096,8098,8100$ and 8219$)$ of founder 8005 . Only one copy of transgene with one integration was detected in 8098 and 8219 (Figure 4-5B) and no rearrangement or deletion detected in 8098 progenies $(8489,8491$ and 8493) (Figure 4-5C). We named the transgenic line established from founder 8005 with on copy of transgene as $8005 \mathrm{~S}$. Finally we generated three independent TetO-sPrdm16 transgenic lines: 8012 8005S (with one copy, no rearrangement) and 8080.

To further identify transgene localization, bone marrow cells from each transgenic line was submitted for FISH. Integration site of transgene in 8012 line was chromosome 19B-C1 region, chromosome $3 \mathrm{C}-\mathrm{D}$ region for $8005 \mathrm{~S}$ line and chromosome $2 \mathrm{C}-1$ region in for 8080 line (Figure 4-6A).

Western blot was carried out on transgenic bone marrow cells to detect sPRDM16 and GFP protein expression. PCR positive transgenic mice and litter mate control were under $2 \mathrm{mg} / \mathrm{ml}$ doxycycline induction for 7 days. Sucrose water without doxycycline was used as control. Due to incomplete induction in bone marrow cells, GFP positive cells were sorted for western blot to increase the sensitivity. Singlets from control bone marrow cells were also sorted for protein detection. sPRDM16 and GFP were only expressed in transgenic bone marrow cells under doxycycline induction while sPRDM16 and GFP was not detected in control (Figure 4-6B) WT bone marrow cells in the presence or absence of doxycycline as well as non-induced transgenic bone marrow cells (Figure 4-6B). This result indicates specificity of gene induction and protein expression in TetO-sPrdm16 transgenic mice.

Transgenic mice from 8012 line were under doxycycline induction for 39 days and bone marrow was analyzed for GFP induction in different lineages. Overall GFP induction showed doxycycline dose-dependent induction in HSC, progenitors, B cell and myeloid cells, but very low induction in MEPs which can't be enhanced by higher dose of doxycycline (Figure 4-7). No GFP was detected in WT bone marrow present or absent of doxycycline (Figure 4-7). $2 \mathrm{mg} / \mathrm{ml}$ doxycycline reached the maximal induction which 
A.

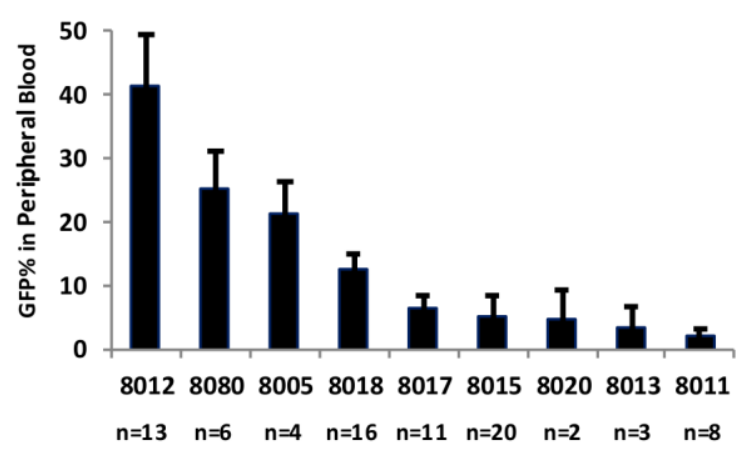

B.

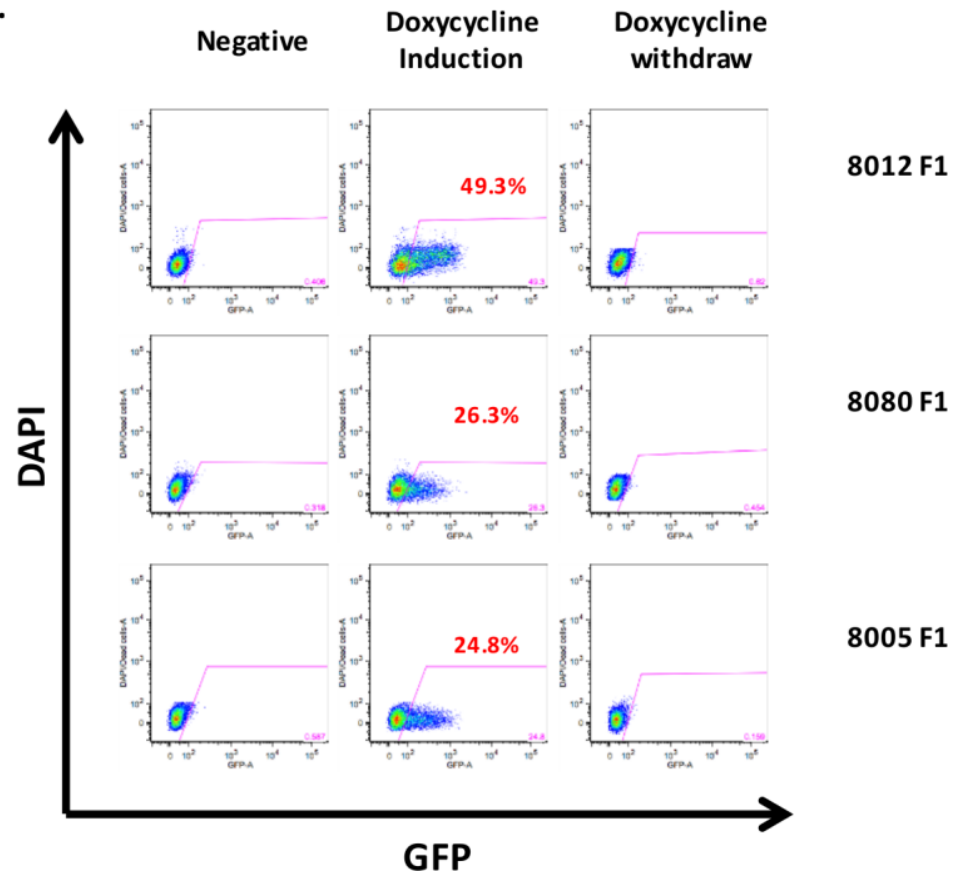

Figure 4-4. GFP expression in peripheral blood of N1 progenies.

(A). Different level of GFP\% of peripheral blood of 9 founders' N1 progenies 7 days post Doxycycline induction in drinking water. X-axis is founder ID and number of N1 of each founder tested. (B). Flow diagram of GFP expression in top 3 GFP induction N1s of each founder. Note GFP expression post doxycycline induction and diminished GFP expression once doxycycline was withdrew for 7 days. 
A.

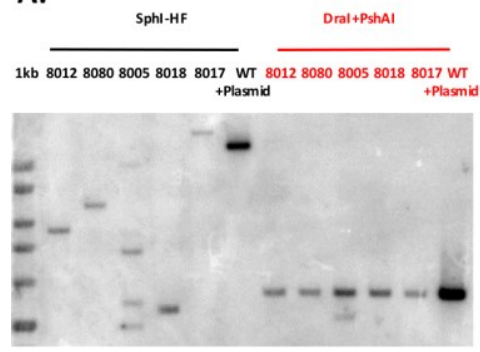

B.

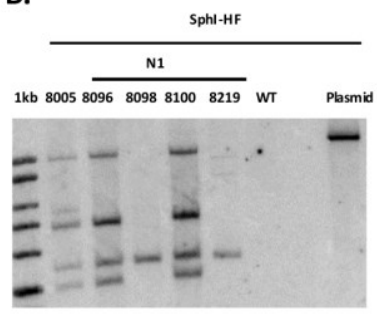

C.

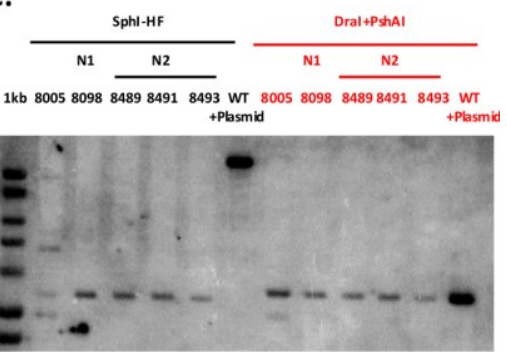

Figure 4-5. Southern blot of TetO-sPrdm16 transgenic mice.

(A). Southern blot on five transgenic founders showed 8005 carried more than one copy of transgene with multiple integrations. Rearrangement or deletion of transgene was also detected in 8005 genome. Other 4 transgenic founders demonstrated single copy of transgene with one integration. No rearrangement or deletion was found in those founders. (B). Southern blot on 8005 and its N1 progenies. Segregation of multiple integration occurred in N1 progenies. 8096 and 8100 carried the same integration pattern as founder 8005 while 8098 and 8219 carried only on copy of transgene with one integration. (C). Southern blot on $8005 \mathrm{~N} 1$ progeny (8098) and N2 progenies $(8489,8491$ and 8493). $\mathrm{N} 2$ progenies inherited transgene integration pattern from 8098. One copy of transgene with one integration was detected in N2 progenies, without rearrangement or deletion. 
A.

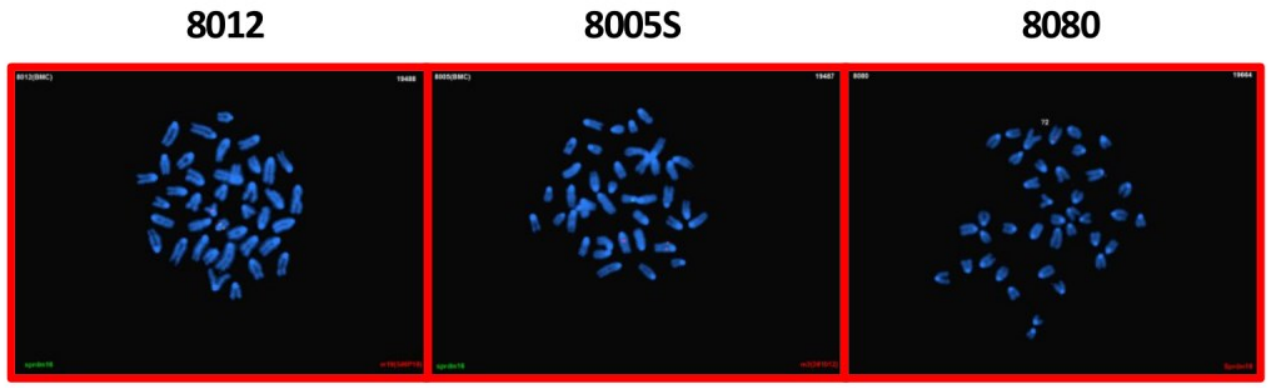

19B-C1

3C-D

2C-1

B.

8012

$8005 S$

8080

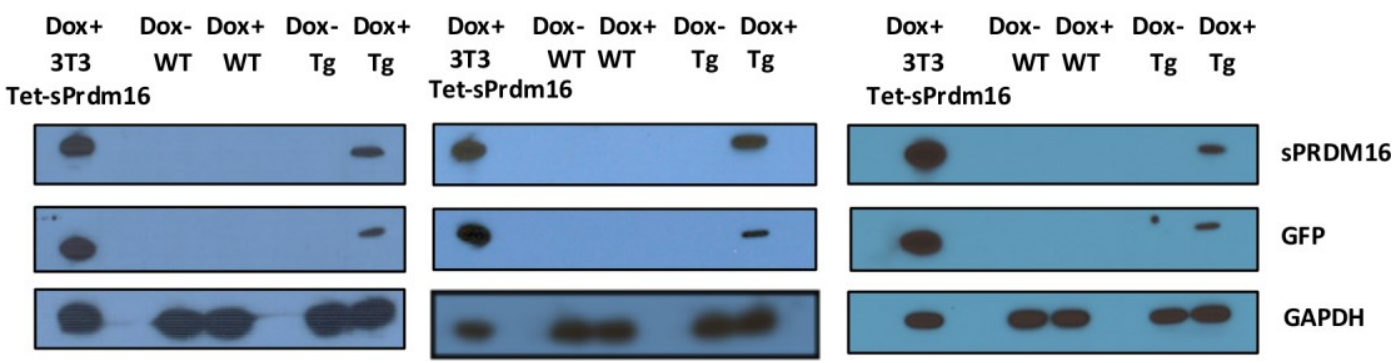

Figure 4-6. TetO-sPrdm16 transgene integration site by FISH and protein expression in transgenic bone marrow cells.

(A). FISH conducted in transgenic mouse bone marrow cells showed transgene localization in mouse genome. Integration site of transgene in 8012 line was chromosome 19B-C1 region, integration site in $8005 \mathrm{~S}$ line was chromosome3C-D region and chromosome $2 \mathrm{C}-1$ region in 8080 line. (B). sPRDM16 and GFP protein expression in transgenic bone marrow cells under the Doxycycline induction in vivo in all three founder lines. No sPRDMd16 and GFP protein expression was detected in wild type bone marrow cells and un-induced transgenic bone marrow cells. GAPDH was used as loading control for western blot. WT: wild type. Tg: Transgenics. Dox: Doxycycline. 
A.

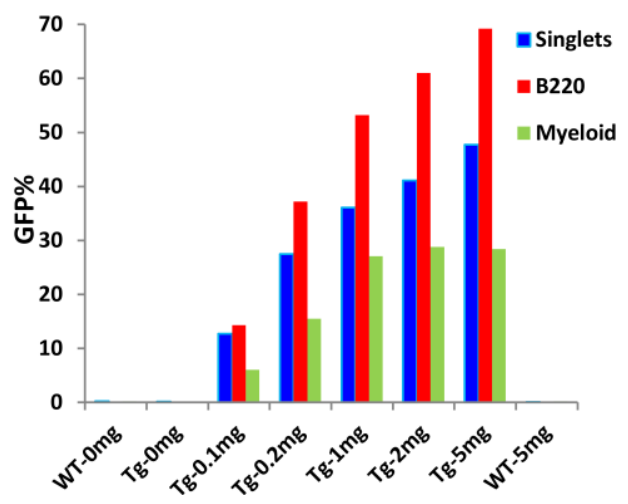

B.

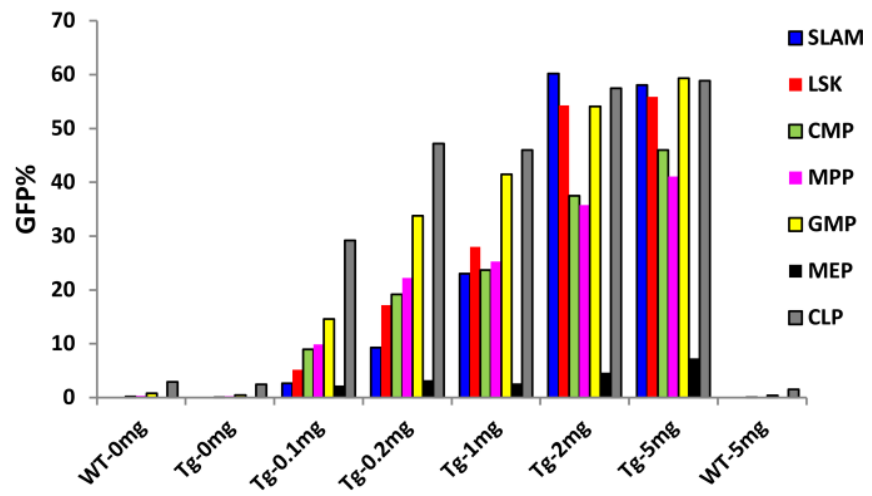

Figure 4-7. GFP percentage in HSC, progenitor and mature lineages in transgenic bone marrow 39 days post doxycycline induction.

(A). GFP percentage in singlets, B cells and Myeloid cells from transgenic bone marrow. (B). GFP percentage in HSC and progenitors from transgenic bone marro.

(A) and (B) both demonstrated doxycycline dose-dependent GFP induction in mature, HSC and progenitor cells. Note low GFP induction in MEPs. 
was not further increased by $5 \mathrm{mg} / \mathrm{ml}$ (Figure 4-7). As a result $2 \mathrm{mg} / \mathrm{ml}$ doxycycline will be used for the future experiment. TetOn-sPrdm16 transgenic mouse model is a useful tool to study role of sPrdm 16 on HSC activity because of efficient induction of sPrdm16 in HSCs.

\section{Overexpression of $s$ Prdm 16 abolishes HOXB4-mediated HSC expansion}

To evaluate $s$ Prdm 16's effect on phonotypical HSC (CD150 ${ }^{+} \mathrm{CD} 48^{-} \mathrm{Sca}-1^{+} \mathrm{c}-$ $\mathrm{Kit}^{+}$Lineage ${ }^{-}$) and MPP (Sca- $1^{+} \mathrm{c}-\mathrm{Kit}^{-}$Lineage ${ }^{-}$) frequency in HOXB4 expressing cells in vivo, bone marrow harvested from 18 weeks' recipients were subjected to SLAM and MPP surface markers staining. mCherry and mCherry + sPrdm 16 transplanted mice served as control. sPRDM16 alone didn't alter MPP frequency compared with mCherry control transduced bone marrow cells. HOXB4 significant increased MPP frequency up to about $5 \%$ of marking cells (Figure 4-8). Different from MPP, HSC frequency was doubled by sPRDM16 and further increased by HOXB4 (Figure4-8). However, sPRDM16 and HOXB4 double positive cells contained less HSCs, similar to control group (Figure 4-8). Results above suggest HOXB4 with or without sPRDM16 expression is able to increase MPP frequency. HOXB4, consistent with previous reports, increased the HSC frequency, which was diminished by sPRDM16 to control level. Lower HSC number but not MPP number implied that sustained expression of sPRDM16 is able to abolish HOXB4mediated HSC expansion but not MPP expansion. However, cell surface marker staining can be altered by certain transcription factor or microRNA expression ${ }^{86}$. The effect of HOXB4 and sPRDM16 on HSC frequency should be further confirmed by limiting dilution assay.

Limiting dilution assay was carried out to assess sPRDM16's effect on functional HSC frequency. Compared with mCherry only group, sPRDM16 didn't significantly alter HSC frequency in recipient mice but HOXB4 recipients had about 10 time more HSCs compared with control (Table 4-1). When sPRDM16 was overexpressed in HOXB4 expressing cells, HSC frequency was reduced about $25 \%$ relative to HOXB4 only group, indicating sustained expression of sPRDM16 in HOXB4 expressing cells indeed reduces HSC frequency and repression of Prdm 16 by HOXB4 is necessary for HOXB4-mediated HSC expansion.

\section{Discussion}

PRDM16 is a hematopoietic stem cell regulator. It plays role in fetal liver HSC and neonatal HSC maintenance. ${ }^{215,216}$ It is preferentially expressed in quiescent LTHSCs, ${ }^{215,216}$ indicating its potential role in maintaining LT-HSC cell at quiescent status. Downregulation of Prdm 16 was observed in HOXB4-expressing HSCs in vivo, raising the hypothesis that HOXB4 downregulates Prdm16 to drive HSCs to enter cell cycle to expand. To test the hypothesis we generated TetOn-sPrdm 16 transgenic mice. Unlike constitutive overexpression vector, stable transgenic mouse model can avoid variation of gene expression and reduce the risk of vector rearrangement due to multiple 

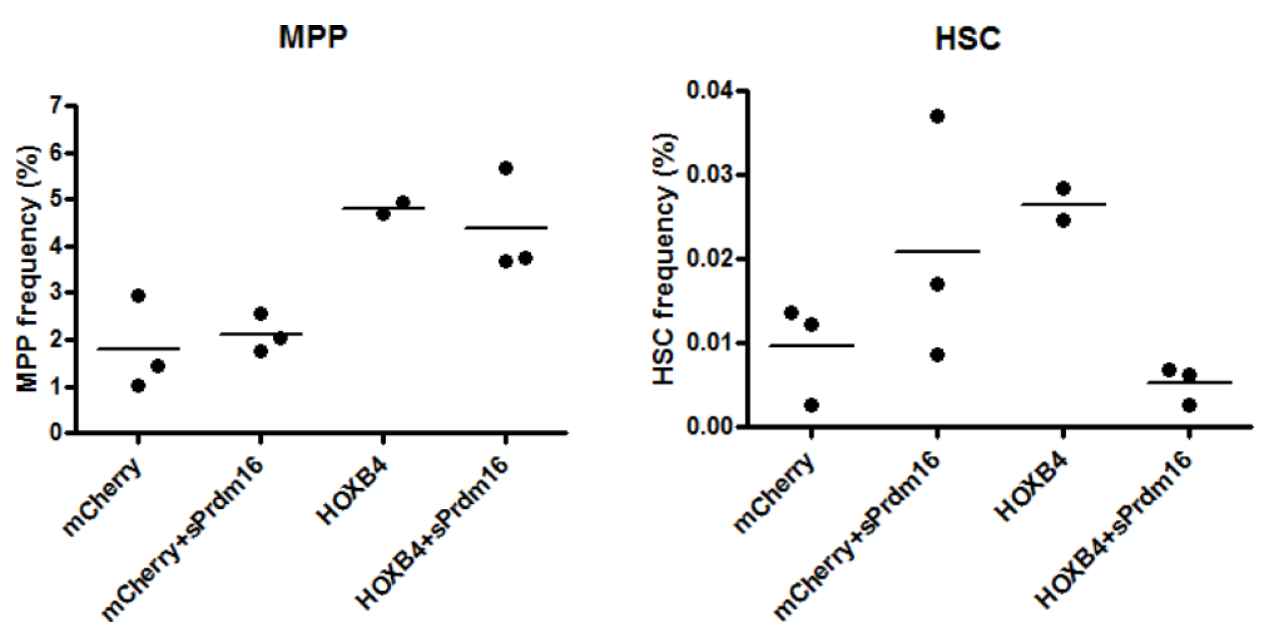

Figure 4-8. MPP and HSC frequency on marking cells.

HOXB4 but not sPrdm16 increases MPP frequency. Sustained expression of sPRDM16 in HOXB4 expressing cells maintain the same MPP frequency as $\mathrm{HOXB}^{+}$cells.

sPRDM16 alone and HOXB4 alone both are able to increase HSC frequency about 2-2.5 fold. Sustained expression of sPRDM16 in HOXB4 expressing cells decreased HSC frequency to control level. 
Table 4-1. HSC frequency in HOXB4 and SPRDM16 transplanted mice.

\begin{tabular}{lcccccc}
\hline Group & Dose & Response & Tested & $\begin{array}{c}\text { HSC } \\
\text { frequency }\end{array}$ & $\begin{array}{c}\text { HSC low } \\
\text { frequency }\end{array}$ & $\begin{array}{c}\text { HSC high } \\
\text { frequency }\end{array}$ \\
\hline mCherry & $2 \mathrm{e} 5$ & 3 & 7 & & & \\
& $4 \mathrm{e} 4$ & 0 & 6 & 1 in & 1 in & 1 in \\
& 8000 & 0 & 7 & 462,515 & $1,416,998$ & 150,967 \\
& 1600 & 0 & 6 & & & \\
mCherry+sPRDM16 & 320 & 0 & 7 & & & \\
& $2 \mathrm{e} 5$ & 3 & 7 & & & 1 in \\
& $4 \mathrm{e} 4$ & 0 & 4 & 1 in & 1 in & \\
& 8000 & 0 & 6 & 431,177 & $1,327,869$ & 140,009 \\
HOXB4 & 1600 & 0 & 4 & & & \\
& 320 & 0 & 7 & & & 1 in \\
& $2 \mathrm{e} 5$ & 7 & 7 & & 1 in & \\
& $4 \mathrm{e} 4$ & 5 & 7 & 1 in & & \\
& 8000 & 0 & 7 & 43,606 & 92,131 & \\
& 1600 & 0 & 6 & & & 1 in \\
& 320 & 0 & 6 & & & 26,256 \\
\hline
\end{tabular}


transductions. CMV promoter incorporated in TetOn construct allows moderate expression of sPRDM16 and may avoid cellular toxicity due to high level of PRDM16 expression driven by MSCV promoter used in previous study. TetOn-sPrdm16 construct allows dose-dependent gene activation by adding different mount of doxycycline, which can prevent cellular toxicity. Expression of GFP was linearly correlated with sPRDM16 expression level due to P2A element between $s$ Prdm 16 and GFP cDNA in the construct, which is usually not seen in IRES containing constructs. As a result, GFP intensity is a great indicator of sPRDM16 expression level. Since vector transduction resulted in limited number of Prdm 16 transduced cells, transgenic mouse model is a great tool for in vivo study as it doesn't require transductions and transgene can be activated in vivo directly.

We generated three TetOn-sPrdm16 transgenic mouse lines. When induced, sPrdm16-GFP showed different induction efficiencies among 3 lines as well as different lineages within each transgenic line. The variation of $s$ Prdm 16 activation efficiency in each line may be due to the unique integration site of plasmid. We observed dosedependent GFP induction in mouse bone marrow as well as PB with best induction in SLAM population and worst in MEPs. Best GFP induction in SLAM cells, which are highly enriched HSCs, is consistent with highest endogenous Prdm 16 expression compared with other lineages. However we noticed poor induction in MEPs though $\mathrm{rtTA}^{\mathrm{M} 2}$ was reported to have best induction efficiency in MEPs compared with other versions of rtTAs. ${ }^{264}$ Low induction efficiency of sPRDM16 on MEPs can be due to the sPRDM16 toxicity on MEPs which led to the elimination of MEPs from bone marrow. Although there was differential expression level of sPRDM16 cross the lineages, high induction efficiency of sPRDM16 on HSCs and progenitors except MEPs makes the TetOn-sPrdm16 transgenic mouse a great tool to study the role of sPRDM16 on HSC's activity.

To address why HOXB4 downregulates Prdm16 in transplanted mice, SLAM frequency and CRU were assessed in HOXB4 transplanted mice under the context of sustained expression of sPRDM16 through doxycycline induction. In HOXB4 only group SLAM frequency and CRU were increased compared to control group. There were reduced SLAM frequency and CRU in HOXB4 and sPRDM16 co-expressing group, indicating sustained expression of sPRDM16 can abolish HOXB4-mediated HSC expansion. Although changes in SLAM frequency and CRU in HOXB4 and SPRDM16 coexpressing group were in the same trend, decrease in CRU was less significant than SLAM frequency. SLAM frequency was calculated on gated HOXB4 and sPRDM16 double positive cells while CRU was assessed on the coexpressing group containing about 50\% HOXB4 single positive cells and only 10-30\% HOXB4 and sPRDM16 double positive cells. With less double positive cells and more HOXB4 cells, effect of double positive cells on CRU could be compensated by HOXB4 single positive cells, which led to less significant changes in CRU than SLAM frequency.

It is striking to detect less HSC when sPRDM16 was expressed in HOXB4 cells as PRDM16 was reported as a critical factor for HSC maintenance. We hypothesize that PRDM16 maintain HSC stemness by maintaining HSC quiescence. HOXB4 represses 
Prdm 16 to release HSC to cell cycle, thus expands HSC in vivo. To test this hypothesis cell cycle status can be evaluated in HOXB4 and sPRDM16 expressing cells from transplanted mice and we expect cell cycle arrest of double positive cells. At meanwhile enhanced apoptosis or downstream differentiation of HSCs also can be the alternative explanation of reduced HSCs by sustained sPRDM16 expression on HOXB4 cells.

In this chapter we generated TetOn-sPrdm16 inducible transgenic mouse model which demonstrated doxycycline dose-dependent sPRDM16/GFP expression in hematopoietic lineages except MEPs. Using this mouse model we showed that sustained expression of sPRDM16 on HOXB4 expressing cells can abolish HOXB4-mediated HSC expansion in transplanted mice. Mechanism how sPRDM16 decreases HSC frequency under the context of HOXB4 overexpression is not included in this chapter but can be explored in the future studies. 


\section{CHAPTER 5. DISCUSSION}

\section{Significance}

The object of this study is to understand molecular mechanism of HOXB4mediated HSC benign expansion in vivo. It started with gene expression array of HOXB4 expressing LSK cells from transplanted mice and then we focused on Prdm16, one of the most significant downregulated genes, to study its role on HOXB4-HSC benign expansion. Results of this study revealed some interesting aspects of HOXB4-mediated HSC expansion.

\section{HOXB4 is a global regulator of HSC self-renewal}

In this study gene expression prolife of HOXB4 expressing LSKs cells from transplanted mice revealed a dynamic transcriptional network of HOXB4 during the reconstitution. In order to expand HSCs, HOXB4 activated hematopoietic stem cell pathway and hematopoietic stem cell long term pathway. Expression of genes regulating HSC function were altered by HOXB4, such as Hemgn, Gata2, N-myc, Pten, Bcl-3, Prdm16, Jun, HOXA9 and many others, which led to balanced HSC expansion. Besides HSC expansion, HOXB4 also facilitate B cell lineage and myeloid lineage priming at LSK cell level. As a result of differentiation permission, HOXB4 is able to control the HSC pool size and prevent the leukemia, a great feature of HOXB4 but not seen in any other oncogenic HOX factors such as HOXA9 and HOXA10. In summary HOXB4 is a global regulator of HSC self-renewal. HOXB4-mediated benign HSC expansion is not achieved by a single downstream target or pathway. It is an outcome of highly complex regulation of genes and pathways playing roles in HSC proliferation, differentiation and apoptosis, which leads to balanced HSC expansion. Besides the knowledge we gained in this study, role of HOXB4 in HSC expansion in vivo is a very interesting topic in the hematology field and worthwhile for further exploration.

\section{Differential regulation of Prdm16 transcript by HOXB4 and HOXA9/A10}

Our study revealed that downregulation of $\operatorname{Prdm} 16$ is unique for HOXB4 but not for HOXA9 and HOXA10 in HSCs. HOXB4, HOXA9 and HOXA10 belong to HOX family. HOX genes all bind to a similar set of 'AT'-rich DNA binding site through their homeodomain and the non-homeodomain adjacent to the homeodomain offer additional HOX-DNA-binding specificity. A bacterial one-hybrid approach $(\mathrm{B} 1 \mathrm{H})$ and in vivo protein-binding microarrays (PBMs) showed that the 'AT'-rich DNA consensus sequences HOXB4 and HOXA9/A10 recognize and bind to are different (Figure 5-1), suggesting HOXB4 and HOXA9/A10 may bind to different region of Prdm 16 promoter

or enhancer. This can be one of the mechanisms of differential transcription regulation of Prdm16 by HOXB4 and HOXA9/A10. There is a study suggesting that HOX gene can 
B1H

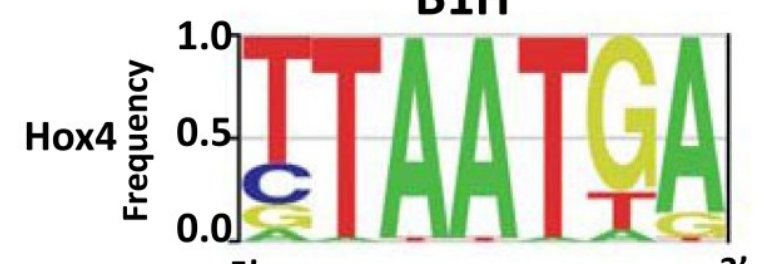

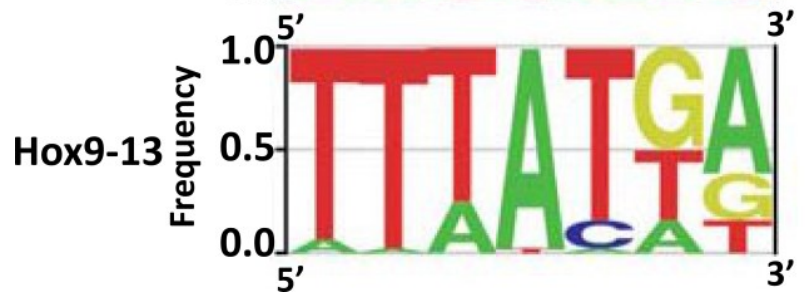

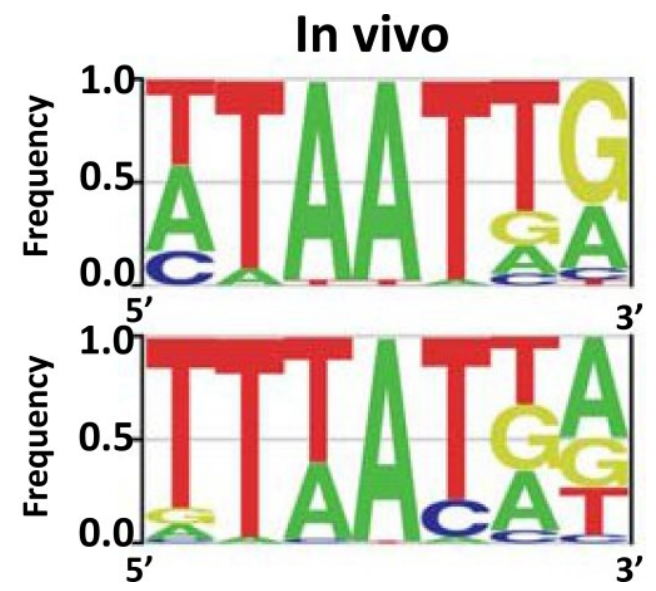

Figure 5-1. Comparison of in vitro and in vivo Hox-binding site preferences. Shown are LOGO diagrams summarizing Hox-binding site preferences for Hox4 and Hox9-13 groups. The column on the left lists the LOGOs generated using the binding sites identified by the $\mathrm{B} 1 \mathrm{H}$ method. The column on the right lists the LOGOs generated using the protein-binding microarrays. Y-axis is nucleotide frequency.

Modified with permission. Mann RS, Lelli KM, Joshi R. Hox specificity unique roles for cofactors and collaborators. Curr Top Dev Biol. 2009;88:63-101. 
use transcription repressors as HOX cofactors in gene repression. Selectively using repressor by HOXB4 but not HOXA9 and HOXA10 can be the explanation of HOXB4mediated Prdm 16 repression. HOX collaborators are another group of proteins which binds to HOX factors or HOX-targeted elements to determine target genes are activated or repressed. Protein-protein interaction between HOX factors and collaborators may form enhanceosome-like structure on $\mathrm{HOX}$-targeted region to activate target genes expression. HOXB4 and HOXA9/A10 may form transcription complex with different collaborators and determine whether to active or repress Prdm16 transcription. ${ }^{114}$

\section{HOXB4 doesn't cause leukemia unless pro-oncogenes are activated}

HOXB4 is always considered as a safe factor to expand HSCs, because overexpression of HOXB4 doesn't lead to hematopoietic malignancies. Safety issue was brought back to discussion when the two myeloid leukemia cases from a dog and a macque who received HOXB4 transduced CD $34^{+}$cells ${ }^{187}$ were reported. In our independent screening we also detected two myeloid leukemias derived from HOXB4 expressing cells in transplanted mice. Both studies revealed additional genetic lesions in leukemic cells due to vector integration and activation of $s$ Prdm 16 was common in both studies, indicating HOXB4 is doesn't transform hematopoietic cells unless additional oncogenes are activated and activation of sPRDM16 is one of the factors contributing to the leukemogenesis. Leukemic cells line generated from the leukemic dog demonstrated sPRDM16-dependent growth in vitro. However, it is possible the leukemias with sPrdm 16 activation may be purely due to sPrdm 16 expression and HOXB4 was not at all involved. In our study we didn't detect any hematopoietic malignancies when $s$ Prdm 16 was overexpressed alone. Shing D.C et al also claimed that $s$ Prdm 16 was not oncogenic in WT mice but in $p 53$ null mice. ${ }^{248}$ Both studies suggest $s$ Prdm 16 alone is not sufficient to cause leukemia. So our screening together with previous literature reports support the synergistic role of HOXB4 and sPRDM16 in leukemogenesis.

\section{Oncogenic potential of sPRDM16}

SPRDM16 is the short form of PRDM16 with partial deletion of N-terminal PR domain. sPRDM16 but not PRDM16 is considered as a proto-oncogene ${ }^{189}$ and is associated human hematopoietic malignancies. Absence of the PR domain may count for the oncogenic potential of sPRDM16. PR domain is 20-30\% identical to SET domain, indicating the potential role of PRDM16 as a methyltransferase. sPRDM16 is highly homologous to EVI1, zinc finger part of MDS1/EVI1 (PRDM3) protein. EVI1 is oncogenic and aberrant expression of EVI1 is detected in about $10 \%$ of AML cases ${ }^{265}$ and is able to induce MDS in mice. ${ }^{266}$ Similar to Prdm16, EVI1 gene expression is upregulated in NUP98-HOXA9-expressing leukemia cells. ${ }^{267}$ Several mechanisms have been identified to contribute to the leukemogenic activity of EVI1 which can be shared by sPRDM16. EVI1 exerts negative regulation on TGF- $\beta$ signaling pathway through the repression of Smad3 function, which promotes cellular proliferation and affects cellular differentiation. EVI1 also suppresses the JNK-1-mediated phosphorylation of c-JUN or 
inhibits interferon- $\alpha$ signaling to exert anti-apoptotic effects. ${ }^{268,269}$ Recent studies showed that EVI recruits and forms protein complex with several Polycomb proteins to PTEN locus and switch PTEN promoter from $\mathrm{H} 3 \mathrm{~K} 27 \mathrm{Ac}$ status to $\mathrm{H} 3 \mathrm{~K} 27 \mathrm{~m} 3$ status to repress Pten expression, which activates PI3K/ARK/Mtorc1 signaling in leukemia cells. ${ }^{270,271}$ Mechanisms of leukemogenic potential of EVI1 mentioned above suggest that EVI1 unique regulation of certain pathways and recruitment of epigenetic modifiers confer EVI1's oncogenic activity and lack of HMT activity from PR domain is not the reason. sPRDM16 can induce leukemia in the similar manner as EVI1 due to the structural and biology similarities between them.

\section{MDS/AML genetic lesion is missing from HOXB4/sPrdm16 preleukemia model}

When sPrdm 16 and HOXB4 were co-overexpressed, only 2 out of 12 recipients developed preluekmias at 28 or 32 weeks post transplantation. Two preleukemic cases represent MDS/AML phenotype with normal karyotype. Low incidence may be due to low cotransduction efficiency. However, the long latency for leukemogenesis indicates activation sPrdm16 and HOXB4 are not sufficient to cause leukemic transformation. MDS/AML is a heterogeneous group of hematopoietic stem cell disorders and acquired mutations such as NPM1 ${ }^{272}$, TET2 $2^{273}$ and FLT3 $3^{274}$ were well characterized in sporadic cases of AML. Submicroscopic deletion of $5 \mathrm{q}$ was recently identified in a patient with MDS presenting with normal karyotype resulting in loss of tumor suppressor CTNNA1 and HSPA9. ${ }^{275}$ ASXL1 frameshift/stop mutation $\left(591^{*}\right), E Z H 2$ point mutation $(\mathrm{R} 690 \mathrm{H})$, RUNX1 frameshift/ stop mutations (c.474dupT and c424_425ins11bp) and TET2 frameshift/stop mutations (c.1510_1513delAAAA and R $1465^{*}$ ) were also detected as MDS-associated mutations. ${ }^{275,276} \bar{R} U N X 1$ mutations are frequently detected in de novo AML with noncomplex karyotype and were proposed as clinical biomarkers for disease progression of MDS to AML. ${ }^{277}$ Harriet Holme et $\mathrm{al}^{278}$ reported genetic categorization of 27 families with familial MDS/AML, revealing heterozygous mutations in the GATA2 gene (c.121C $>$ G p.Pro41Ala; c1187G $>$ A p. Arg396Gln and c1061C $>$ T, p.Thr354Met), TERT mutations, TERC mutations and RUNX1 mutation. There are also MDS/AML cases with unidentified mutations. One or many of the mutations mentioned above may be required for the complete MDS/AML transformation in our HOXB4 and sPrdm16 transplanted animals, missing which led to delayed onset of the leukemias.

\section{Repression of Prdm16 may promote cell enter cell cycle and facilitate HOXB4- mediated HSC expansion}

Expansion of HSCs requires HSCs to enter cell cycle. PRDM16 shared high similarity of MDS1/EVI1 protein, suggesting the PRDM16 may share similar mechanism how MDS1/EVI1 regulate HSC self-renewal can. MDS1/EVI1 was shown to be negative cell cycle regulator, loss of which led to increased proportion of active dividing HSCs and HSC exhaustion. ${ }^{218}$ Endogenous Prdm 16 is highly expressed in quiescent LT-HSCs and loss of Prdm 16 increased the proportion of HSCs undergoing active cell

division. $^{215,216}$ We detected marked repression of Prdm 16 in HOXB4-HSCs. It is 
possible that HOXB4 represses Prdm 16 to release HSCs from cell cycle arrest state. When $s$ Prdm 16 expression is sustained in HOXB4 expressing cells, HSC proliferation can be suppressed, which results in reduced phonotypical and functional HSCs number observed in this study, supporting the hypothesis that repressing Prdm 16 in HOXB4 expressing cells is required for HOXB4-mediated HSC expansion. Loss of Prdm 16 also induces apoptosis/cell death in HSCs. ${ }^{215,216}$ Repression of Prdm 16 by HOXB4 can be a mechanism to induced apoptosis in unwanted proliferating HSCs, which is contradict to our previous finding that HOXB4 indeed protects hematopoietic progenitors from apoptosis. Although how HOXB4 downregulates Prdm 16 in transplanted mice is unknown, but it is clear sustained expression of $s P r d m 16$ decreased HSC frequency in HOXB4 transplanted mice. In conclusion, repression of Prdm 16 is required for HOXB4mediated HSC expansion in vivo.

\section{Future Directions}

HOXB4-mediated HSC expansion is an interesting topic. Findings of our study revealed the unique regulation of Prdm 16 by HOXB4 during HSC expansion in vivo. Considering the limitations discussed above, several experiments can be carried out to improve this study.

Our study showed significant downregulation of Prdm 16 by HOXB4 in transplanted mice. To address the question how HOXB4 downregulates Prdm16, ChIP assay can be performed. We have generated HOXB4-dependent myeloid cell line. This cell line contains $\mathrm{Grl}^{-} \mathrm{Mac}^{-}{ }^{-} \mathrm{Fc} \mathrm{R}^{-}$primitive population which demonstrated HOXB4dependent proliferation and myeloid progenitor self-renewal capacity. We have conducted ChIP assay using I12 HOXB4 monoclonal antibody and HOXB4 protein enrichment was nicely achieved. However, ChIP-seq data showed low signal-to-noise ratio which makes peak calling impossible. High noise can be due to low specificity of the HOXB4 antibody or non-stringent washing condition, both can be improve by changing to a ChIP-grade antibody and more stringent washing solution. But non-specific binding of HOXB4 to mouse genome due to high expression level of HOXB4 by overexpression vector can also contribute to the high noise. Lower the protein expression by switching to weak promoter probably can decrease the noise.

As mentioned above another question is not answered by our study is how downregulation of $\operatorname{Prdm} 16$ contributes to the HSC expansion in HOXB4 transplanted mice. Given the fact that loss of Prdm 16 release HSC into cell cycle, ${ }^{215,216}$ repression of Prdm 16 can set HSC at active proliferation state, thus allowing HSC expansion. To test the hypothesis, cell cycle analysis can be done on HSC compartment of HOXB4 and sPRDM16 co-expressing cells and we predict higher proportion of G0 cells in HOXB4 and sPRDM16 double positive HSCs than HOXB4 single positive HSCs.

$\operatorname{Prdm} 16$ was claimed as a hematopoietic stem cell regulator. ${ }^{216}$ Mechanistic studies regarding its role in hematopoietic specification and HSC maintenance are lacking. Numerous studies have identified molecular mechanism of Prdm 16-mediated 
brown fat maturation, including complex elements, upstream regulators and downstream pathways. Prdm 16 was also reported to maintain heterochromatin integrity through its H3K9me1 methyltransferase activity. As one of the long-term directions, molecular interaction of Prdm16 with other factors and involvement of Prdm16 in epigenetic modification during embryonic and adult hematopoiesis can be explored, findings of which will provide great insight of HSC biology.

Our study showed that oncogenic HOXA9 and HOXA10 upregulated Prdm16 up to about 50 fold compared with HOXB4 in HSC compartment, suggesting aberrant expression of Prdm 16 could be a major player in HOXA9 or HOXA10-associated leukemia initiation or maintenance. If so, overexpression of HOXA9/10 in Prdm16 null HSCs but not WT HSCs will not lead to leukemia. It will be exciting to confirm the role of Prdm 16 and identify Prdm 16 pathway in HOXA9/10-asscoiated leukemia. Drug screening can be conducted to identify promising compounds targeting Prdm16 pathway associated with HOXA9/10 leukemia. 


\section{LIST OF REFERENCES}

1. Morrison SJ, Weissman IL. The long-term repopulating subset of hematopoietic stem cells is deterministic and isolatable by phenotype. Immunity. 1994;1(8):661673.

2. Christensen JL, Weissman IL. Flk-2 is a marker in hematopoietic stem cell differentiation: a simple method to isolate long-term stem cells. Proc Natl Acad Sci U S A. 2001;98(25):14541-14546.

3. Kondo M, Weissman IL, Akashi K. Identification of clonogenic common lymphoid progenitors in mouse bone marrow. Cell. 1997;91(5):661-672.

4. Akashi K, Traver D, Miyamoto T, Weissman IL. A clonogenic common myeloid progenitor that gives rise to all myeloid lineages. Nature. 2000;404(6774):193197.

5. Bell JJ, Bhandoola A. The earliest thymic progenitors for T cells possess myeloid lineage potential. Nature. 2008;452(7188):764-767.

6. Guo G, Luc S, Marco E, et al. Mapping cellular hierarchy by single-cell analysis of the cell surface repertoire. Cell Stem Cell. 2013;13(4):492-505.

7. Adolfsson J, Mansson R, Buza-Vidas N, et al. Identification of Flt3+ lymphomyeloid stem cells lacking erythro-megakaryocytic potential a revised road map for adult blood lineage commitment. Cell. 2005;121(2):295-306.

8. Pronk CJ, Rossi DJ, Mansson R, et al. Elucidation of the phenotypic, functional, and molecular topography of a myeloerythroid progenitor cell hierarchy. Cell Stem Cell. 2007;1(4):428-442.

9. Medvinsky AL, Samoylina NL, Muller AM, Dzierzak EA. An early pre-liver intraembryonic source of CFU-S in the developing mouse. Nature. 1993;364(6432):64-67.

10. Medvinsky A, Dzierzak E. Definitive hematopoiesis is autonomously initiated by the AGM region. Cell. 1996;86(6):897-906.

11. Muller AM, Medvinsky A, Strouboulis J, Grosveld F, Dzierzak E. Development of hematopoietic stem cell activity in the mouse embryo. Immunity. 1994;1(4):291-301.

12. Larsson J, Karlsson S. The role of Smad signaling in hematopoiesis. Oncogene. 2005;24(37):5676-5692.

13. Boisset JC, van Cappellen W, Andrieu-Soler C, Galjart N, Dzierzak E, Robin C. In vivo imaging of haematopoietic cells emerging from the mouse aortic endothelium. Nature. 2010;464(7285):116-120.

14. Bertrand JY, Chi NC, Santoso B, Teng S, Stainier DY, Traver D. Haematopoietic stem cells derive directly from aortic endothelium during development. Nature. 2010;464(7285):108-111.

15. Till JE, Mc CE. A direct measurement of the radiation sensitivity of normal mouse bone marrow cells. Radiat Res. 1961;14:213-222.

16. Iscove NN, Till JE, McCulloch EA. The proliferative states of mouse granulopoietic progenitor cells. Proc Soc Exp Biol Med. 1970;134(1):33-36. 
17. Iscove NN, Messner H, Till JE, McCulloch EA. Human marrow cells forming colonies in culture: analysis by velocity sedimentation and suspension culture. Ser Haematol. 1972;5(2):37-49.

18. Wu AM, Till JE, Siminovitch L, McCulloch EA. A cytological study of the capacity for differentiation of normal hemopoietic colony-forming cells. J Cell Physiol. 1967;69(2):177-184.

19. Morrison SJ, Kimble J. Asymmetric and symmetric stem-cell divisions in development and cancer. Nature. 2006;441(7097):1068-1074.

20. Sekulovic S, Gylfadottir V, Vulto I, et al. Prolonged self-renewal activity unmasks telomerase control of telomere homeostasis and function of mouse hematopoietic stem cells. Blood. 2011;118(7):1766-1773.

21. Morrison SJ, Prowse KR, Ho P, Weissman IL. Telomerase activity in hematopoietic cells is associated with self-renewal potential. Immunity. 1996;5(3):207-216.

22. Allsopp RC, Cheshier S, Weissman IL. Telomere shortening accompanies increased cell cycle activity during serial transplantation of hematopoietic stem cells. J Exp Med. 2001;193(8):917-924.

23. Dzierzak E, Speck NA. Of lineage and legacy: the development of mammalian hematopoietic stem cells. Nat Immunol. 2008;9(2):129-136.

24. Fleischman RA, Custer RP, Mintz B. Totipotent hematopoietic stem cells: normal self-renewal and differentiation after transplantation between mouse fetuses. Cell. 1982;30(2):351-359.

25. Lemischka IR, Raulet DH, Mulligan RC. Developmental potential and dynamic behavior of hematopoietic stem cells. Cell. 1986;45(6):917-927.

26. Harrison DE, Astle CM, Lerner C. Number and continuous proliferative pattern of transplanted primitive immunohematopoietic stem cells. Proc Natl Acad Sci US A. 1988;85(3):822-826.

27. Spangrude GJ, Heimfeld S, Weissman IL. Purification and characterization of mouse hematopoietic stem cells. Science. 1988;241(4861):58-62.

28. Jordan CT, Lemischka IR. Clonal and systemic analysis of long-term hematopoiesis in the mouse. Genes Dev. 1990;4(2):220-232.

29. Smith LG, Weissman IL, Heimfeld S. Clonal analysis of hematopoietic stem-cell differentiation in vivo. Proc Natl Acad Sci U S A. 1991;88(7):2788-2792.

30. Osawa M, Hanada K, Hamada H, Nakauchi H. Long-term lymphohematopoietic reconstitution by a single CD34-low/negative hematopoietic stem cell. Science. 1996;273(5272):242-245.

31. Ikuta K, Weissman IL. Evidence that hematopoietic stem cells express mouse ckit but do not depend on steel factor for their generation. Proc Natl Acad Sci US A. 1992;89(4):1502-1506.

32. Okada S, Nakauchi H, Nagayoshi K, Nishikawa S, Miura Y, Suda T. In vivo and in vitro stem cell function of c-kit- and Sca-1-positive murine hematopoietic cells. Blood. 1992;80(12):3044-3050.

33. Orlic D, Fischer R, Nishikawa S, Nienhuis AW, Bodine DM. Purification and characterization of heterogeneous pluripotent hematopoietic stem cell populations expressing high levels of c-kit receptor. Blood. 1993;82(3):762-770. 
34. Baum CM, Weissman IL, Tsukamoto AS, Buckle AM, Peault B. Isolation of a candidate human hematopoietic stem-cell population. Proc Natl Acad Sci U S A. 1992;89(7):2804-2808.

35. Matsuoka S, Ebihara Y, Xu M, et al. CD34 expression on long-term repopulating hematopoietic stem cells changes during developmental stages. Blood. 2001;97(2):419-425.

36. Kiel MJ, Yilmaz OH, Iwashita T, Yilmaz OH, Terhorst C, Morrison SJ. SLAM family receptors distinguish hematopoietic stem and progenitor cells and reveal endothelial niches for stem cells. Cell. 2005;121(7):1109-1121.

37. Goodell MA, Brose K, Paradis G, Conner AS, Mulligan RC. Isolation and functional properties of murine hematopoietic stem cells that are replicating in vivo. J Exp Med. 1996;183(4):1797-1806.

38. Zhou S, Schuetz JD, Bunting KD, et al. The ABC transporter Bcrp1/ABCG2 is expressed in a wide variety of stem cells and is a molecular determinant of the side-population phenotype. Nat Med. 2001;7(9):1028-1034.

39. Fleming WH, Alpern EJ, Uchida N, Ikuta K, Spangrude GJ, Weissman IL. Functional heterogeneity is associated with the cell cycle status of murine hematopoietic stem cells. J Cell Biol. 1993;122(4):897-902.

40. Harrison DE. Competitive repopulation: a new assay for long-term stem cell functional capacity. Blood. 1980;55(1):77-81.

41. Szilvassy SJ, Humphries RK, Lansdorp PM, Eaves AC, Eaves CJ. Quantitative assay for totipotent reconstituting hematopoietic stem cells by a competitive repopulation strategy. Proc Natl Acad Sci U S A. 1990;87(22):8736-8740.

42. Ratajczak MZ. Phenotypic and functional characterization of hematopoietic stem cells. Curr Opin Hematol. 2008;15(4):293-300.

43. Tsai FY, Orkin SH. Transcription factor GATA-2 is required for proliferation/survival of early hematopoietic cells and mast cell formation, but not for erythroid and myeloid terminal differentiation. Blood. 1997;89(10):36363643.

44. Tsai FY, Keller G, Kuo FC, et al. An early haematopoietic defect in mice lacking the transcription factor GATA-2. Nature. 1994;371(6494):221-226.

45. Minegishi N, Suzuki N, Yokomizo T, et al. Expression and domain-specific function of GATA-2 during differentiation of the hematopoietic precursor cells in midgestation mouse embryos. Blood. 2003;102(3):896-905.

46. Tipping AJ, Pina C, Castor A, et al. High GATA-2 expression inhibits human hematopoietic stem and progenitor cell function by effects on cell cycle. Blood. 2009;113(12):2661-2672.

47. Tripic T, Deng W, Cheng Y, et al. SCL and associated proteins distinguish active from repressive GATA transcription factor complexes. Blood. 2009;113(10):2191-2201.

48. Park IK, Qian D, Kiel M, et al. Bmi-1 is required for maintenance of adult selfrenewing haematopoietic stem cells. Nature. 2003;423(6937):302-305.

49. Iwama A, Oguro H, Negishi M, et al. Enhanced self-renewal of hematopoietic stem cells mediated by the polycomb gene product Bmi-1. Immunity. 2004;21(6):843-851. 
50. Rizo A, Dontje B, Vellenga E, de Haan G, Schuringa JJ. Long-term maintenance of human hematopoietic stem/progenitor cells by expression of BMI1. Blood. 2008;111(5):2621-2630.

51. Rizo A, Olthof S, Han L, Vellenga E, de Haan G, Schuringa JJ. Repression of BMI1 in normal and leukemic human CD34(+) cells impairs self-renewal and induces apoptosis. Blood. 2009;114(8):1498-1505.

52. Molofsky AV, Pardal R, Iwashita T, Park IK, Clarke MF, Morrison SJ. Bmi-1 dependence distinguishes neural stem cell self-renewal from progenitor proliferation. Nature. 2003;425(6961):962-967.

53. Ohta H, Sawada A, Kim JY, et al. Polycomb group gene rae 28 is required for sustaining activity of hematopoietic stem cells. $J$ Exp Med. 2002;195(6):759-770.

54. Alkema MJ, Bronk M, Verhoeven E, et al. Identification of Bmil-interacting proteins as constituents of a multimeric mammalian polycomb complex. Genes Dev. 1997;11(2):226-240.

55. Gunster MJ, Satijn DP, Hamer KM, et al. Identification and characterization of interactions between the vertebrate polycomb-group protein BMI1 and human homologs of polyhomeotic. Mol Cell Biol. 1997;17(4):2326-2335.

56. Hashimoto N, Brock HW, Nomura M, et al. RAE28, BMI1, and M33 are members of heterogeneous multimeric mammalian Polycomb group complexes. Biochem Biophys Res Commun. 1998;245(2):356-365.

57. Tomotsune D, Takihara Y, Berger J, et al. A novel member of murine Polycombgroup proteins, Sex comb on midleg homolog protein, is highly conserved, and interacts with RAE28/mph1 in vitro. Differentiation. 1999;65(4):229-239.

58. Shao Z, Raible F, Mollaaghababa R, et al. Stabilization of chromatin structure by PRC1, a Polycomb complex. Cell. 1999;98(1):37-46.

59. Cales C, Roman-Trufero M, Pavon L, et al. Inactivation of the polycomb group protein Ring1B unveils an antiproliferative role in hematopoietic cell expansion and cooperation with tumorigenesis associated with Ink4a deletion. Mol Cell Biol. 2008;28(3):1018-1028.

60. Lessard J, Schumacher A, Thorsteinsdottir U, van Lohuizen M, Magnuson T, Sauvageau G. Functional antagonism of the Polycomb-Group genes eed and Bmil in hemopoietic cell proliferation. Genes Dev. 1999;13(20):2691-2703.

61. Micklem HS, Ford CE, Evans EP, Ogden DA, Papworth DS. Competitive in vivo proliferation of foetal and adult haematopoietic cells in lethally irradiated mice. $J$ Cell Physiol. 1972;79(2):293-298.

62. Majewski IJ, Blewitt ME, de Graaf CA, et al. Polycomb repressive complex 2 (PRC2) restricts hematopoietic stem cell activity. PLoS Biol. 2008;6(4):e93.

63. Tadokoro Y, Ema H, Okano M, Li E, Nakauchi H. De novo DNA methyltransferase is essential for self-renewal, but not for differentiation, in hematopoietic stem cells. $J$ Exp Med. 2007;204(4):715-722.

64. Challen GA, Sun D, Jeong M, et al. Dnmt3a is essential for hematopoietic stem cell differentiation. Nat Genet. 2012;44(1):23-31.

65. Trowbridge JJ, Snow JW, Kim J, Orkin SH. DNA methyltransferase 1 is essential for and uniquely regulates hematopoietic stem and progenitor cells. Cell Stem Cell. 2009;5(4):442-449. 
66. Trowbridge JJ, Sinha AU, Zhu N, Li M, Armstrong SA, Orkin SH. Haploinsufficiency of Dnmt1 impairs leukemia stem cell function through derepression of bivalent chromatin domains. Genes Dev. 2012;26(4):344-349.

67. Broske AM, Vockentanz L, Kharazi S, et al. DNA methylation protects hematopoietic stem cell multipotency from myeloerythroid restriction. Nat Genet. 2009;41(11):1207-1215.

68. Austin TW, Solar GP, Ziegler FC, Liem L, Matthews W. A role for the Wnt gene family in hematopoiesis: expansion of multilineage progenitor cells. Blood. 1997;89(10):3624-3635.

69. Brandon C, Eisenberg LM, Eisenberg CA. WNT signaling modulates the diversification of hematopoietic cells. Blood. 2000;96(13):4132-4141.

70. Reya T, Duncan AW, Ailles L, et al. A role for Wnt signalling in self-renewal of haematopoietic stem cells. Nature. 2003;423(6938):409-414.

71. Chung EJ, Hwang SG, Nguyen P, et al. Regulation of leukemic cell adhesion, proliferation, and survival by beta-catenin. Blood. 2002;100(3):982-990.

72. Xue Y, Gao X, Lindsell CE, et al. Embryonic lethality and vascular defects in mice lacking the Notch ligand Jagged1. Hum Mol Genet. 1999;8(5):723-730.

73. Walker L, Lynch M, Silverman S, et al. The Notch/Jagged pathway inhibits proliferation of human hematopoietic progenitors in vitro. Stem Cells. 1999;17(3):162-171.

74. Ohishi K, Varnum-Finney B, Bernstein ID. Delta-1 enhances marrow and thymus repopulating ability of human $\mathrm{CD} 34(+) \mathrm{CD} 38(-)$ cord blood cells. J Clin Invest. 2002;110(8):1165-1174.

75. Varnum-Finney B, Xu L, Brashem-Stein C, et al. Pluripotent, cytokinedependent, hematopoietic stem cells are immortalized by constitutive Notch1 signaling. Nat Med. 2000;6(11):1278-1281.

76. Groszer M, Erickson R, Scripture-Adams DD, et al. Negative regulation of neural stem/progenitor cell proliferation by the Pten tumor suppressor gene in vivo.

Science. 2001;294(5549):2186-2189.

77. Groszer M, Erickson R, Scripture-Adams DD, et al. PTEN negatively regulates neural stem cell self-renewal by modulating G0-G1 cell cycle entry. Proc Natl Acad Sci U S A. 2006;103(1):111-116.

78. Gregorian C, Nakashima J, Le Belle J, et al. Pten deletion in adult neural stem/progenitor cells enhances constitutive neurogenesis. J Neurosci. 2009;29(6):1874-1886.

79. Yilmaz $\mathrm{OH}$, Valdez R, Theisen BK, et al. Pten dependence distinguishes haematopoietic stem cells from leukaemia-initiating cells. Nature. 2006;441(7092):475-482.

80. Zhang J, Grindley JC, Yin T, et al. PTEN maintains haematopoietic stem cells and acts in lineage choice and leukaemia prevention. Nature. 2006;441(7092):518-522.

81. Winnier G, Blessing M, Labosky PA, Hogan BL. Bone morphogenetic protein-4 is required for mesoderm formation and patterning in the mouse. Genes Dev. 1995;9(17):2105-2116. 
82. Bhatia $\mathrm{M}$, Bonnet $\mathrm{D}, \mathrm{Wu} \mathrm{D}$, et al. Bone morphogenetic proteins regulate the developmental program of human hematopoietic stem cells. J Exp Med. 1999;189(7):1139-1148.

83. Bhardwaj G, Murdoch B, Wu D, et al. Sonic hedgehog induces the proliferation of primitive human hematopoietic cells via BMP regulation. Nat Immunol. 2001;2(2):172-180.

84. Goey H, Keller JR, Back T, Longo DL, Ruscetti FW, Wiltrout RH. Inhibition of early murine hemopoietic progenitor cell proliferation after in vivo locoregional administration of transforming growth factor-beta 1. J Immunol. 1989;143(3):877880.

85. Blank U, Karlsson G, Moody JL, et al. Smad7 promotes self-renewal of hematopoietic stem cells. Blood. 2006;108(13):4246-4254.

86. Guo S, Lu J, Schlanger R, et al. MicroRNA miR-125a controls hematopoietic stem cell number. Proc Natl Acad Sci U S A. 2010;107(32):14229-14234.

87. Georgantas RW, 3rd, Hildreth R, Morisot S, et al. CD34+ hematopoietic stemprogenitor cell microRNA expression and function: a circuit diagram of differentiation control. Proc Natl Acad Sci U S A. 2007;104(8):2750-2755.

88. Petriv OI, Kuchenbauer F, Delaney AD, et al. Comprehensive microRNA expression profiling of the hematopoietic hierarchy. Proc Natl Acad Sci U S A. 2010;107(35):15443-15448.

89. O'Connell RM, Chaudhuri AA, Rao DS, Gibson WS, Balazs AB, Baltimore D. MicroRNAs enriched in hematopoietic stem cells differentially regulate long-term hematopoietic output. Proc Natl Acad Sci U S A. 2010;107(32):14235-14240.

90. Gentner B, Visigalli I, Hiramatsu H, et al. Identification of hematopoietic stem cell-specific miRNAs enables gene therapy of globoid cell leukodystrophy. Sci Transl Med. 2010;2(58):58ra84.

91. Bousquet M, Quelen C, Rosati R, et al. Myeloid cell differentiation arrest by miR$125 \mathrm{~b}-1$ in myelodysplastic syndrome and acute myeloid leukemia with the $\mathrm{t}(2 ; 11)(\mathrm{p} 21 ; \mathrm{q} 23)$ translocation. $J$ Exp Med. 2008;205(11):2499-2506.

92. Chapiro E, Russell LJ, Struski S, et al. A new recurrent translocation $\mathrm{t}(11 ; 14)(\mathrm{q} 24 ; \mathrm{q} 32)$ involving IGH@ and miR-125b-1 in B-cell progenitor acute lymphoblastic leukemia. Leukemia. 2010;24(7):1362-1364.

93. Sonoki T, Iwanaga E, Mitsuya H, Asou N. Insertion of microRNA-125b-1, a human homologue of lin-4, into a rearranged immunoglobulin heavy chain gene locus in a patient with precursor B-cell acute lymphoblastic leukemia. Leukemia. 2005;19(11):2009-2010.

94. Calin GA, Croce CM. Chronic lymphocytic leukemia: interplay between noncoding RNAs and protein-coding genes. Blood. 2009;114(23):4761-4770.

95. O'Connell RM, Rao DS, Chaudhuri AA, et al. Sustained expression of microRNA-155 in hematopoietic stem cells causes a myeloproliferative disorder. J Exp Med. 2008;205(3):585-594.

96. Thai TH, Calado DP, Casola S, et al. Regulation of the germinal center response by microRNA-155. Science. 2007;316(5824):604-608.

97. Fazi F, Rosa A, Fatica A, et al. A minicircuitry comprised of microRNA-223 and transcription factors NFI-A and C/EBPalpha regulates human granulopoiesis.

Cell. 2005;123(5):819-831. 
98. Johnnidis JB, Harris MH, Wheeler RT, et al. Regulation of progenitor cell proliferation and granulocyte function by microRNA-223. Nature. 2008;451(7182):1125-1129.

99. Chen CZ, Li L, Lodish HF, Bartel DP. MicroRNAs modulate hematopoietic lineage differentiation. Science. 2004;303(5654):83-86.

100. Xiao C, Calado DP, Galler G, et al. MiR-150 controls B cell differentiation by targeting the transcription factor c-Myb. Cell. 2007;131(1):146-159.

101. Xiao C, Srinivasan L, Calado DP, et al. Lymphoproliferative disease and autoimmunity in mice with increased miR-17-92 expression in lymphocytes. Nat Immunol. 2008;9(4):405-414.

102. Zhou B, Wang S, Mayr C, Bartel DP, Lodish HF. miR-150, a microRNA expressed in mature $\mathrm{B}$ and $\mathrm{T}$ cells, blocks early $\mathrm{B}$ cell development when expressed prematurely. Proc Natl Acad Sci U S A. 2007;104(17):7080-7085.

103. Akam M. Hox and HOM: homologous gene clusters in insects and vertebrates. Cell. 1989;57(3):347-349.

104. Kaufman TC, Seeger MA, Olsen G. Molecular and genetic organization of the antennapedia gene complex of Drosophila melanogaster. Adv Genet. 1990;27:309-362.

105. Duncan I. The bithorax complex. Annu Rev Genet. 1987;21:285-319.

106. Mazo AM, Huang DH, Mozer BA, Dawid IB. The trithorax gene, a trans-acting regulator of the bithorax complex in Drosophila, encodes a protein with zincbinding domains. Proc Natl Acad Sci U S A. 1990;87(6):2112-2116.

107. Alharbi RA, Pettengell R, Pandha HS, Morgan R. The role of HOX genes in normal hematopoiesis and acute leukemia. Leukemia. 2013;27(5):1000-1008.

108. Kaneko Y, Shikano T, Maseki N, et al. Clinical characteristics of infant acute leukemia with or without 11q23 translocations. Leukemia. 1988;2(10):672-676.

109. Hunger SP, Tkachuk DC, Amylon MD, et al. HRX involvement in de novo and secondary leukemias with diverse chromosome 11q23 abnormalities. Blood. 1993;81(12):3197-3203.

110. Yu BD, Hess JL, Horning SE, Brown GA, Korsmeyer SJ. Altered Hox expression and segmental identity in Mll-mutant mice. Nature. 1995;378(6556):505-508.

111. Yagi H, Deguchi K, Aono A, Tani Y, Kishimoto T, Komori T. Growth disturbance in fetal liver hematopoiesis of Mll-mutant mice. Blood. 1998;92(1):108-117.

112. Lessard J, Baban S, Sauvageau G. Stage-specific expression of polycomb group genes in human bone marrow cells. Blood. 1998;91(4):1216-1224.

113. Moens CB, Selleri L. Hox cofactors in vertebrate development. Dev Biol. 2006;291(2):193-206.

114. Mann RS, Lelli KM, Joshi R. Hox specificity unique roles for cofactors and collaborators. Curr Top Dev Biol. 2009;88:63-101.

115. Mann RS. The specificity of homeotic gene function. Bioessays. 1995;17(10):855-863.

116. Shen WF, Montgomery JC, Rozenfeld S, et al. AbdB-like Hox proteins stabilize DNA binding by the Meis1 homeodomain proteins. Mol Cell Biol. 1997; 17(11):6448-6458. 
117. Chang CP, Brocchieri L, Shen WF, Largman C, Cleary ML. Pbx modulation of Hox homeodomain amino-terminal arms establishes different DNA-binding specificities across the Hox locus. Mol Cell Biol. 1996;16(4):1734-1745.

118. Neuteboom ST, Peltenburg LT, van Dijk MA, Murre C. The hexapeptide LFPWMR in Hoxb-8 is required for cooperative DNA binding with Pbx 1 and Pbx2 proteins. Proc Natl Acad Sci U S A. 1995;92(20):9166-9170.

119. Phelan ML, Rambaldi I, Featherstone MS. Cooperative interactions between HOX and PBX proteins mediated by a conserved peptide motif. Mol Cell Biol. 1995;15(8):3989-3997.

120. Lu Q, Kamps MP. Selective repression of transcriptional activators by Pbx1 does not require the homeodomain. Proc Natl Acad Sci U S A. 1996;93(1):470-474.

121. Shen WF, Chang CP, Rozenfeld S, et al. Hox homeodomain proteins exhibit selective complex stabilities with Pbx and DNA. Nucleic Acids Res. 1996;24(5):898-906.

122. Shen WF, Rozenfeld S, Lawrence HJ, Largman C. The Abd-B-like Hox homeodomain proteins can be subdivided by the ability to form complexes with Pbxla on a novel DNA target. J Biol Chem. 1997;272(13):8198-8206.

123. Moskow JJ, Bullrich F, Huebner K, Daar IO, Buchberg AM. Meis1, a PBX1related homeobox gene involved in myeloid leukemia in BXH-2 mice. Mol Cell Biol. 1995;15(10):5434-5443.

124. Chang CP, Jacobs Y, Nakamura T, Jenkins NA, Copeland NG, Cleary ML. Meis proteins are major in vivo DNA binding partners for wild-type but not chimeric Pbx proteins. Mol Cell Biol. 1997;17(10):5679-5687.

125. Knoepfler PS, Kamps MP. The highest affinity DNA element bound by Pbx complexes in $\mathrm{t}(1 ; 19)$ leukemic cells fails to mediate cooperative DNA-binding or cooperative transactivation by E2a-Pbx1 and class I Hox proteins - evidence for selective targetting of E2a-Pbx1 to a subset of Pbx-recognition elements. Oncogene. 1997;14(21):2521-2531.

126. Nakamura T, Jenkins NA, Copeland NG. Identification of a new family of Pbxrelated homeobox genes. Oncogene. 1996;13(10):2235-2242.

127. Steelman S, Moskow JJ, Muzynski K, et al. Identification of a conserved family of Meis1-related homeobox genes. Genome Res. 1997;7(2):142-156.

128. Berthelsen J, Zappavigna V, Mavilio F, Blasi F. Prep1, a novel functional partner of Pbx proteins. EMBO J. 1998;17(5):1423-1433.

129. Berthelsen J, Zappavigna V, Ferretti E, Mavilio F, Blasi F. The novel homeoprotein Prep1 modulates Pbx-Hox protein cooperativity. EMBO J. 1998;17(5):1434-1445.

130. Shen WF, Rozenfeld S, Kwong A, Kom ves LG, Lawrence HJ, Largman C. HOXA9 forms triple complexes with PBX2 and MEIS1 in myeloid cells. Mol Cell Biol. 1999;19(4):3051-3061.

131. Giampaolo A, Sterpetti P, Bulgarini D, et al. Key functional role and lineagespecific expression of selected HOXB genes in purified hematopoietic progenitor differentiation. Blood. 1994;84(11):3637-3647. 
132. Kawagoe H, Humphries RK, Blair A, Sutherland HJ, Hogge DE. Expression of HOX genes, HOX cofactors, and MLL in phenotypically and functionally defined subpopulations of leukemic and normal human hematopoietic cells. Leukemia. 1999;13(5):687-698.

133. Pineault N, Helgason CD, Lawrence HJ, Humphries RK. Differential expression of Hox, Meis1, and Pbx 1 genes in primitive cells throughout murine hematopoietic ontogeny. Exp Hematol. 2002;30(1):49-57.

134. Sauvageau G, Lansdorp PM, Eaves CJ, et al. Differential expression of homeobox genes in functionally distinct CD34+ subpopulations of human bone marrow cells. Proc Natl Acad Sci U S A. 1994;91(25):12223-12227.

135. Crooks GM, Fuller J, Petersen D, et al. Constitutive HOXA5 expression inhibits erythropoiesis and increases myelopoiesis from human hematopoietic progenitors. Blood. 1999;94(2):519-528.

136. Fuller JF, McAdara J, Yaron Y, Sakaguchi M, Fraser JK, Gasson JC. Characterization of HOX gene expression during myelopoiesis: role of HOX A5 in lineage commitment and maturation. Blood. 1999;93(10):3391-3400.

137. So CW, Karsunky H, Wong P, Weissman IL, Cleary ML. Leukemic transformation of hematopoietic progenitors by MLL-GAS7 in the absence of Hoxa7 or Hoxa9. Blood. 2004;103(8):3192-3199.

138. Thorsteinsdottir U, Mamo A, Kroon E, et al. Overexpression of the myeloid leukemia-associated Hoxa9 gene in bone marrow cells induces stem cell expansion. Blood. 2002;99(1):121-129.

139. Kroon E, Krosl J, Thorsteinsdottir U, Baban S, Buchberg AM, Sauvageau G. Hoxa9 transforms primary bone marrow cells through specific collaboration with Meis1a but not Pbx1b. EMBO J. 1998;17(13):3714-3725.

140. Buske C, Feuring-Buske M, Antonchuk J, et al. Overexpression of HOXA10 perturbs human lymphomyelopoiesis in vitro and in vivo. Blood. 2001;97(8):2286-2292.

141. Sauvageau G, Thorsteinsdottir U, Eaves CJ, et al. Overexpression of HOXB4 in hematopoietic cells causes the selective expansion of more primitive populations in vitro and in vivo. Genes Dev. 1995;9(14):1753-1765.

142. Bijl J, Thompson A, Ramirez-Solis R, et al. Analysis of HSC activity and compensatory Hox gene expression profile in Hoxb cluster mutant fetal liver cells. Blood. 2006;108(1):116-122.

143. Sauvageau G, Thorsteinsdottir U, Hough MR, et al. Overexpression of HOXB3 in hematopoietic cells causes defective lymphoid development and progressive myeloproliferation. Immunity. 1997;6(1):13-22.

144. Ko KH, Lam QL, Zhang M, et al. Hoxb3 deficiency impairs B lymphopoiesis in mouse bone marrow. Exp Hematol. 2007;35(3):465-475.

145. Shimamoto T, Tang Y, Naot Y, et al. Hematopoietic progenitor cell abnormalities in Hoxc-8 null mutant mice. J Exp Zool. 1999;283(2):186-193.

146. Kappen C. Disruption of the homeobox gene Hoxb-6 in mice results in increased numbers of early erythrocyte progenitors. Am J Hematol. 2000;65(2):111-118. 
147. Fischbach NA, Rozenfeld S, Shen W, et al. HOXB6 overexpression in murine bone marrow immortalizes a myelomonocytic precursor in vitro and causes hematopoietic stem cell expansion and acute myeloid leukemia in vivo. Blood. 2005;105(4):1456-1466.

148. Daga A, Podesta M, Capra MC, Piaggio G, Frassoni F, Corte G. The retroviral transduction of HOXC4 into human CD34(+) cells induces an in vitro expansion of clonogenic and early progenitors. Exp Hematol. 2000;28(5):569-574.

149. Takeshita K, Bollekens JA, Hijiya N, Ratajczak M, Ruddle FH, Gewirtz AM. A homeobox gene of the Antennapedia class is required for human adult erythropoiesis. Proc Natl Acad Sci U S A. 1993;90(8):3535-3538.

150. Magnusson M, Brun AC, Lawrence HJ, Karlsson S. Hoxa9/hoxb3/hoxb4 compound null mice display severe hematopoietic defects. Exp Hematol. 2007;35(9):1421-1428.

151. Amsellem S, Pflumio F, Bardinet D, et al. Ex vivo expansion of human hematopoietic stem cells by direct delivery of the HOXB4 homeoprotein. Nat Med. 2003;9(11):1423-1427.

152. Brun AC, Bjornsson JM, Magnusson M, et al. Hoxb4-deficient mice undergo normal hematopoietic development but exhibit a mild proliferation defect in hematopoietic stem cells. Blood. 2004;103(11):4126-4133.

153. Bjornsson JM, Larsson N, Brun AC, et al. Reduced proliferative capacity of hematopoietic stem cells deficient in Hoxb3 and Hoxb4. Mol Cell Biol. 2003;23(11):3872-3883.

154. Borrow J, Shearman AM, Stanton VP, Jr., et al. The t(7;11)(p15;p15) translocation in acute myeloid leukaemia fuses the genes for nucleoporin NUP98 and class I homeoprotein HOXA9. Nat Genet. 1996;12(2):159-167.

155. Nakamura T, Largaespada DA, Lee MP, et al. Fusion of the nucleoporin gene NUP98 to HOXA9 by the chromosome translocation $\mathrm{t}(7 ; 11)(\mathrm{p} 15 ; \mathrm{p} 15)$ in human myeloid leukaemia. Nat Genet. 1996;12(2):154-158.

156. Fujino T, Suzuki A, Ito Y, et al. Single-translocation and double-chimeric transcripts: detection of NUP98-HOXA9 in myeloid leukemias with HOXA11 or HOXA13 breaks of the chromosomal translocation $\mathrm{t}(7 ; 11)(\mathrm{p} 15 ; \mathrm{p} 15)$. Blood. 2002;99(4):1428-1433.

157. Suzuki A, Ito Y, Sashida G, et al. $\mathrm{t}(7 ; 11)(\mathrm{p} 15 ; \mathrm{p} 15)$ Chronic myeloid leukaemia developed into blastic transformation showing a novel NUP98/HOXA11 fusion. Br J Haematol. 2002;116(1):170-172.

158. Taketani T, Taki T, Shibuya N, et al. The HOXD11 gene is fused to the NUP98 gene in acute myeloid leukemia with $\mathrm{t}(2 ; 11)(\mathrm{q} 31 ; \mathrm{p} 15)$. Cancer Res. 2002;62(1):33-37.

159. Raza-Egilmez SZ, Jani-Sait SN, Grossi M, Higgins MJ, Shows TB, Aplan PD. NUP98-HOXD13 gene fusion in therapy-related acute myelogenous leukemia. Cancer Res. 1998;58(19):4269-4273.

160. Taketani T, Taki T, Shibuya N, Kikuchi A, Hanada R, Hayashi Y. Novel NUP98$\mathrm{HOXC1} 1$ fusion gene resulted from a chromosomal break within exon 1 of HOXC11 in acute myeloid leukemia with $\mathrm{t}(11 ; 12)(\mathrm{p} 15 ; \mathrm{q} 13)$. Cancer Res. 2002;62(16):4571-4574. 
161. Slape C, Aplan PD. The role of NUP98 gene fusions in hematologic malignancy. Leuk Lymphoma. 2004;45(7):1341-1350.

162. Pineault N, Buske C, Feuring-Buske M, et al. Induction of acute myeloid leukemia in mice by the human leukemia-specific fusion gene NUP98-HOXD13 in concert with Meis1. Blood. 2003;101(11):4529-4538.

163. Kroon E, Thorsteinsdottir U, Mayotte N, Nakamura T, Sauvageau G. NUP98HOXA9 expression in hemopoietic stem cells induces chronic and acute myeloid leukemias in mice. EMBO J. 2001;20(3):350-361.

164. Dash AB, Williams IR, Kutok JL, et al. A murine model of CML blast crisis induced by cooperation between BCR/ABL and NUP98/HOXA9. Proc Natl Acad Sci U S A. 2002;99(11):7622-7627.

165. Milne TA, Briggs SD, Brock HW, et al. MLL targets SET domain methyltransferase activity to Hox gene promoters. Mol Cell. 2002;10(5):11071117.

166. Slany RK. The molecular biology of mixed lineage leukemia. Haematologica. 2009;94(7):984-993.

167. Ayton PM, Cleary ML. Transformation of myeloid progenitors by MLL oncoproteins is dependent on Hoxa7 and Hoxa9. Genes Dev. 2003;17(18):22982307.

168. Wang J, Iwasaki H, Krivtsov A, et al. Conditional MLL-CBP targets GMP and models therapy-related myeloproliferative disease. EMBO J. 2005;24(2):368-381.

169. Kumar AR, Hudson WA, Chen W, Nishiuchi R, Yao Q, Kersey JH. Hoxa9 influences the phenotype but not the incidence of Mll-AF9 fusion gene leukemia. Blood. 2004;103(5): 1823-1828.

170. Rawat VP, Thoene S, Naidu VM, et al. Overexpression of CDX2 perturbs HOX gene expression in murine progenitors depending on its $\mathrm{N}$-terminal domain and is closely correlated with deregulated HOX gene expression in human acute myeloid leukemia. Blood. 2008;111(1):309-319.

171. Falini B, Bolli N, Liso A, et al. Altered nucleophosmin transport in acute myeloid leukaemia with mutated NPM1: molecular basis and clinical implications.

Leukemia. 2009;23(10):1731-1743.

172. Mullighan CG, Kennedy A, Zhou X, et al. Pediatric acute myeloid leukemia with NPM1 mutations is characterized by a gene expression profile with dysregulated HOX gene expression distinct from MLL-rearranged leukemias. Leukemia. 2007;21(9):2000-2009.

173. Lawrence HJ, Largman C. Homeobox genes in normal hematopoiesis and leukemia. Blood. 1992;80(10):2445-2453.

174. Hills D, Gribi R, Ure J, et al. Hoxb4-YFP reporter mouse model: a novel tool for tracking HSC development and studying the role of Hoxb4 in hematopoiesis. Blood. 2011;117(13):3521-3528.

175. Helgason CD, Sauvageau G, Lawrence HJ, Largman C, Humphries RK. Overexpression of HOXB4 enhances the hematopoietic potential of embryonic stem cells differentiated in vitro. Blood. 1996;87(7):2740-2749.

176. Kyba M, Perlingeiro RC, Daley GQ. HoxB4 confers definitive lymphoid-myeloid engraftment potential on embryonic stem cell and yolk sac hematopoietic progenitors. Cell. 2002;109(1):29-37. 
177. Chan KM, Bonde S, Klump H, Zavazava N. Hematopoiesis and immunity of HOXB4-transduced embryonic stem cell-derived hematopoietic progenitor cells. Blood. 2008;111(6):2953-2961.

178. Bowles KM, Vallier L, Smith JR, Alexander MR, Pedersen RA. HOXB4 overexpression promotes hematopoietic development by human embryonic stem cells. Stem Cells. 2006;24(5):1359-1369.

179. Lu SJ, Feng Q, Ivanova Y, et al. Recombinant HoxB4 fusion proteins enhance hematopoietic differentiation of human embryonic stem cells. Stem Cells Dev. 2007;16(4):547-559.

180. Lee GS, Kim BS, Sheih JH, Moore M. Forced expression of HoxB4 enhances hematopoietic differentiation by human embryonic stem cells. Mol Cells. 2008;25(4):487-493.

181. Thorsteinsdottir U, Sauvageau G, Humphries RK. Enhanced in vivo regenerative potential of HOXB4-transduced hematopoietic stem cells with regulation of their pool size. Blood. 1999;94(8):2605-2612.

182. Antonchuk J, Sauvageau G, Humphries RK. HOXB4 overexpression mediates very rapid stem cell regeneration and competitive hematopoietic repopulation. Exp Hematol. 2001;29(9):1125-1134.

183. Antonchuk J, Sauvageau G, Humphries RK. HOXB4-induced expansion of adult hematopoietic stem cells ex vivo. Cell. 2002;109(1):39-45.

184. Buske C, Feuring-Buske M, Abramovich C, et al. Deregulated expression of HOXB4 enhances the primitive growth activity of human hematopoietic cells. Blood. 2002;100(3):862-868.

185. Zhang XB, Schwartz JL, Humphries RK, Kiem HP. Effects of HOXB4 overexpression on ex vivo expansion and immortalization of hematopoietic cells from different species. Stem Cells. 2007;25(8):2074-2081.

186. Lee J, Shieh JH, Zhang J, et al. Improved ex vivo expansion of adult hematopoietic stem cells by overcoming CUL4-mediated degradation of HOXB4. Blood. 2013.

187. Zhang XB, Beard BC, Trobridge GD, et al. High incidence of leukemia in large animals after stem cell gene therapy with a HOXB4-expressing retroviral vector. $J$ Clin Invest. 2008;118(4):1502-1510.

188. Fumasoni I, Meani N, Rambaldi D, Scafetta G, Alcalay M, Ciccarelli FD. Family expansion and gene rearrangements contributed to the functional specialization of PRDM genes in vertebrates. BMC Evol Biol. 2007;7:187.

189. Kinameri E, Inoue T, Aruga J, et al. Prdm proto-oncogene transcription factor family expression and interaction with the Notch-Hes pathway in mouse neurogenesis. PLoS One. 2008;3(12):e3859.

190. Derunes C, Briknarova K, Geng L, et al. Characterization of the PR domain of RIZ1 histone methyltransferase. Biochem Biophys Res Commun. 2005;333(3):925-934.

191. Eom GH, Kim K, Kim SM, et al. Histone methyltransferase PRDM8 regulates mouse testis steroidogenesis. Biochem Biophys Res Commun. 2009;388(1):131136. 
192. Hayashi K, Yoshida K, Matsui Y. A histone H3 methyltransferase controls epigenetic events required for meiotic prophase. Nature. 2005;438(7066):374378.

193. Fruhbeck G, Sesma P, Burrell MA. PRDM16: the interconvertible adipo-myocyte switch. Trends Cell Biol. 2009;19(4):141-146.

194. Kim KC, Geng L, Huang S. Inactivation of a histone methyltransferase by mutations in human cancers. Cancer Res. 2003;63(22):7619-7623.

195. Endo K, Karim MR, Taniguchi H, et al. Chromatin modification of Notch targets in olfactory receptor neuron diversification. Nat Neurosci. 2012;15(2):224-233.

196. Izutsu K, Kurokawa M, Imai Y, Maki K, Mitani K, Hirai H. The corepressor CtBP interacts with Evi-1 to repress transforming growth factor beta signaling. Blood. 2001;97(9):2815-2822.

197. Kajimura S, Seale P, Tomaru T, et al. Regulation of the brown and white fat gene programs through a PRDM16/CtBP transcriptional complex. Genes Dev. 2008;22(10):1397-1409.

198. Nishikata I, Nakahata S, Saito Y, et al. Sumoylation of MEL1S at lysine 568 and its interaction with CtBP facilitates its repressor activity and the blockade of GCSF-induced myeloid differentiation. Oncogene. 2011;30(40):4194-4207.

199. Palmer S, Brouillet JP, Kilbey A, et al. Evi-1 transforming and repressor activities are mediated by CtBP co-repressor proteins. J Biol Chem. 2001;276(28):2583425840.

200. Quinlan KG, Nardini M, Verger A, et al. Specific recognition of ZNF217 and other zinc finger proteins at a surface groove of $\mathrm{C}$-terminal binding proteins. $\mathrm{Mol}$ Cell Biol. 2006;26(21):8159-8172.

201. Van Campenhout C, Nichane M, Antoniou A, et al. Evi1 is specifically expressed in the distal tubule and duct of the Xenopus pronephros and plays a role in its formation. Dev Biol. 2006;294(1):203-219.

202. Kuo TC, Calame KL. B lymphocyte-induced maturation protein (Blimp)-1, IFN regulatory factor (IRF)-1, and IRF-2 can bind to the same regulatory sites. $J$ Immunol. 2004;173(9):5556-5563.

203. Delwel R, Funabiki T, Kreider BL, Morishita K, Ihle JN. Four of the seven zinc fingers of the Evi-1 myeloid-transforming gene are required for sequence-specific binding to GA(C/T)AAGA(T/C)AAGATAA. Mol Cell Biol. 1993;13(7):42914300 .

204. Funabiki T, Kreider BL, Ihle JN. The carboxyl domain of zinc fingers of the Evi1 myeloid transforming gene binds a consensus sequence of GAAGATGAG. Oncogene. 1994;9(6):1575-1581.

205. Brzezinski JAt, Lamba DA, Reh TA. Blimp1 controls photoreceptor versus bipolar cell fate choice during retinal development. Development. 2010;137(4):619-629.

206. Katoh K, Omori Y, Onishi A, Sato S, Kondo M, Furukawa T. Blimp1 suppresses Chx10 expression in differentiating retinal photoreceptor precursors to ensure proper photoreceptor development. J Neurosci. 2010;30(19):6515-6526.

207. Wilm TP, Solnica-Krezel L. Essential roles of a zebrafish prdm1/blimp1 homolog in embryo patterning and organogenesis. Development. 2005;132(2):393-404. 
208. Harper J, Mould A, Andrews RM, Bikoff EK, Robertson EJ. The transcriptional repressor Blimp1/Prdm1 regulates postnatal reprogramming of intestinal enterocytes. Proc Natl Acad Sci U S A. 2011;108(26):10585-10590.

209. Muncan V, Heijmans J, Krasinski SD, et al. Blimp1 regulates the transition of neonatal to adult intestinal epithelium. Nat Commun. 2011;2:452.

210. Shapiro-Shelef M, Lin KI, Savitsky D, Liao J, Calame K. Blimp-1 is required for maintenance of long-lived plasma cells in the bone marrow. J Exp Med. 2005;202(11):1471-1476.

211. Yu J, Angelin-Duclos C, Greenwood J, Liao J, Calame K. Transcriptional repression by blimp-1 (PRDI-BF1) involves recruitment of histone deacetylase. Mol Cell Biol. 2000;20(7):2592-2603.

212. Su ST, Ying HY, Chiu YK, Lin FR, Chen MY, Lin KI. Involvement of histone demethylase LSD1 in Blimp-1-mediated gene repression during plasma cell differentiation. Mol Cell Biol. 2009;29(6):1421-1431.

213. Seale P, Kajimura S, Yang W, et al. Transcriptional control of brown fat determination by PRDM16. Cell Metab. 2007;6(1):38-54.

214. Seale P, Bjork B, Yang W, et al. PRDM16 controls a brown fat/skeletal muscle switch. Nature. 2008;454(7207):961-967.

215. Chuikov S, Levi BP, Smith ML, Morrison SJ. Prdm16 promotes stem cell maintenance in multiple tissues, partly by regulating oxidative stress. Nat Cell Biol. 2010;12(10):999-1006.

216. Aguilo F, Avagyan S, Labar A, et al. Prdm16 is a physiologic regulator of hematopoietic stem cells. Blood. 2011;117(19):5057-5066.

217. Goyama S, Yamamoto G, Shimabe M, et al. Evi-1 is a critical regulator for hematopoietic stem cells and transformed leukemic cells. Cell Stem Cell. 2008;3(2):207-220.

218. Zhang Y, Stehling-Sun S, Lezon-Geyda K, et al. PR-domain-containing Mds1Evi1 is critical for long-term hematopoietic stem cell function. Blood. 2011;118(14):3853-3861.

219. Morishita K, Parganas E, William CL, et al. Activation of EVI1 gene expression in human acute myelogenous leukemias by translocations spanning 300-400 kilobases on chromosome band 3q26. Proc Natl Acad Sci U S A. 1992;89(9):3937-3941.

220. Suzukawa K, Kodera T, Shimizu S, et al. Activation of EVI1 transcripts with chromosomal translocation joining the TCRVbeta locus and the EVI1 gene in human acute undifferentiated leukemia cell line (Kasumi-3) with a complex translocation of der(3)t(3;7;8). Leukemia. 1999;13(9):1359-1366.

221. Yufu Y, Sadamura S, Ishikura H, et al. Expression of EVI1 and the Retinoblastoma genes in acute myelogenous leukemia with $\mathrm{t}(3 ; 13)(\mathrm{q} 26 ; \mathrm{q} 13-14)$. Am J Hematol. 1996;53(1):30-34.

222. Storlazzi CT, Anelli L, Albano F, et al. A novel chromosomal translocation $\mathrm{t}(3 ; 7)(\mathrm{q} 26 ; \mathrm{q} 21)$ in myeloid leukemia resulting in overexpression of EVI1. Ann Hematol. 2004;83(2):78-83.

223. Yoshida M, Nosaka K, Yasunaga J, Nishikata I, Morishita K, Matsuoka M. Aberrant expression of the MEL1S gene identified in association with hypomethylation in adult T-cell leukemia cells. Blood. 2004;103(7):2753-2760. 
224. Sakai I, Tamura T, Narumi H, et al. Novel RUNX1-PRDM16 fusion transcripts in a patient with acute myeloid leukemia showing $\mathrm{t}(1 ; 21)(\mathrm{p} 36 ; \mathrm{q} 22)$. Genes Chromosomes Cancer. 2005;44(3):265-270.

225. Stevens-Kroef MJ, Schoenmakers EF, van Kraaij M, et al. Identification of truncated RUNX1 and RUNX1-PRDM16 fusion transcripts in a case of $\mathrm{t}(1 ; 21)(\mathrm{p} 36 ; \mathrm{q} 22)-$ positive therapy-related AML. Leukemia. 2006;20(6):11871189.

226. Ott MG, Schmidt M, Schwarzwaelder K, et al. Correction of X-linked chronic granulomatous disease by gene therapy, augmented by insertional activation of MDS1-EVI1, PRDM16 or SETBP1. Nat Med. 2006;12(4):401-409.

227. Du Y, Jenkins NA, Copeland NG. Insertional mutagenesis identifies genes that promote the immortalization of primary bone marrow progenitor cells. Blood. 2005;106(12):3932-3939.

228. Ohinata Y, Ohta H, Shigeta M, Yamanaka K, Wakayama T, Saitou M. A signaling principle for the specification of the germ cell lineage in mice. Cell. 2009;137(3):571-584.

229. Ohinata Y, Payer B, O'Carroll D, et al. Blimp1 is a critical determinant of the germ cell lineage in mice. Nature. 2005;436(7048):207-213.

230. Vincent SD, Dunn NR, Sciammas R, et al. The zinc finger transcriptional repressor Blimp $1 / \mathrm{Prdm} 1$ is dispensable for early axis formation but is required for specification of primordial germ cells in the mouse. Development. 2005;132(6):1315-1325.

231. Yamaji M, Seki Y, Kurimoto K, et al. Critical function of Prdm14 for the establishment of the germ cell lineage in mice. Nat Genet. 2008;40(8):1016-1022.

232. Chia NY, Chan YS, Feng B, et al. A genome-wide RNAi screen reveals determinants of human embryonic stem cell identity. Nature. 2010;468(7321):316-320.

233. Ma Z, Swigut T, Valouev A, Rada-Iglesias A, Wysocka J. Sequence-specific regulator Prdm14 safeguards mouse ESCs from entering extraembryonic endoderm fates. Nat Struct Mol Biol. 2011;18(2):120-127.

234. Tsuneyoshi N, Sumi T, Onda H, Nojima H, Nakatsuji N, Suemori H. PRDM14 suppresses expression of differentiation marker genes in human embryonic stem cells. Biochem Biophys Res Commun. 2008;367(4):899-905.

235. Nishikawa N, Toyota M, Suzuki H, et al. Gene amplification and overexpression of PRDM14 in breast cancers. Cancer Res. 2007;67(20):9649-9657.

236. Dettman EJ, Simko SJ, Ayanga B, et al. Prdm14 initiates lymphoblastic leukemia after expanding a population of cells resembling common lymphoid progenitors. Oncogene. 2011;30(25):2859-2873.

237. Mochizuki N, Shimizu S, Nagasawa T, et al. A novel gene, MEL1, mapped to 1p36.3 is highly homologous to the MDS1/EVI1 gene and is transcriptionally activated in $\mathrm{t}(1 ; 3)(\mathrm{p} 36 ; \mathrm{q} 21)$-positive leukemia cells. Blood. 2000;96(9):32093214.

238. Nishikata I, Sasaki H, Iga M, et al. A novel EVI1 gene family, MEL1, lacking a PR domain (MEL1S) is expressed mainly in $\mathrm{t}(1 ; 3)(\mathrm{p} 36 ; \mathrm{q} 21)$-positive AML and blocks G-CSF-induced myeloid differentiation. Blood. 2003;102(9):3323-3332. 
239. Xiao Z, Zhang M, Liu X, Zhang Y, Yang L, Hao Y. MEL1S, not MEL1, is overexpressed in myelodysplastic syndromes patients with $\mathrm{t}(1 ; 3)(\mathrm{p} 36 ; \mathrm{q} 21)$. Leuk Res. 2006;30(3):332-334.

240. Modlich U, Schambach A, Brugman MH, et al. Leukemia induction after a single retroviral vector insertion in Evil or Prdm16. Leukemia. 2008;22(8):1519-1528.

241. Horn KH, Warner DR, Pisano M, Greene RM. PRDM16 expression in the developing mouse embryo. Acta Histochem. 2011;113(2):150-155.

242. Warner DR, Horn KH, Mudd L, Webb CL, Greene RM, Pisano MM. PRDM16/MEL1: a novel Smad binding protein expressed in murine embryonic orofacial tissue. Biochim Biophys Acta. 2007;1773(6):814-820.

243. Kajimura S, Seale P, Kubota K, et al. Initiation of myoblast to brown fat switch by a PRDM16-C/EBP-beta transcriptional complex. Nature. 2009;460(7259):1154-1158.

244. Ohno H, Shinoda K, Ohyama K, Sharp LZ, Kajimura S. EHMT1 controls brown adipose cell fate and thermogenesis through the PRDM16 complex. Nature. 2013;504(7478):163-167.

245. Pinheiro I, Margueron R, Shukeir N, et al. Prdm3 and Prdm16 are H3K9me1 Methyltransferases Required for Mammalian Heterochromatin Integrity. Cell. 2012;150(5):948-960.

246. Deneault E, Cellot S, Faubert A, et al. A functional screen to identify novel effectors of hematopoietic stem cell activity. Cell. 2009;137(2):369-379.

247. Hazourli S, Chagnon P, Sauvageau M, Fetni R, Busque L, Hebert J. Overexpression of PRDM16 in the presence and absence of the RUNX1/PRDM16 fusion gene in myeloid leukemias. Genes Chromosomes Cancer. 2006;45(11):1072-1076.

248. Shing DC, Trubia M, Marchesi F, et al. Overexpression of sPRDM16 coupled with loss of p53 induces myeloid leukemias in mice. $J$ Clin Invest. 2007;117(12):3696-3707.

249. Satoh Y, Matsumura I, Tanaka H, et al. Roles for c-Myc in self-renewal of hematopoietic stem cells. J Biol Chem. 2004;279(24):24986-24993.

250. Schiedlmeier B, Santos AC, Ribeiro A, et al. HOXB4's road map to stem cell expansion. Proc Natl Acad Sci U S A. 2007;104(43):16952-16957.

251. Jiang J, Yu H, Shou Y, et al. Hemgn is a direct transcriptional target of HOXB4 and induces expansion of murine myeloid progenitor cells. Blood. 2010;116(5):711-719.

252. Lee HM, Zhang H, Schulz V, Tuck DP, Forget BG. Downstream targets of HOXB4 in a cell line model of primitive hematopoietic progenitor cells. Blood. 2010;116(5):720-730.

253. Benjamini Y, Hochberg Y. Controlling the False Discovery Rate: A Practical and Powerful Approach to Multiple Testing. J R Stat Soc B. 1995;57(1).

254. Subramanian A, Tamayo P, Mootha VK, et al. Gene set enrichment analysis: a knowledge-based approach for interpreting genome-wide expression profiles. Proc Natl Acad Sci U S A. 2005;102(43):15545-15550.

255. Ivanova NB, Dimos JT, Schaniel C, Hackney JA, Moore KA, Lemischka IR. A stem cell molecular signature. Science. 2002;298(5593):601-604. 
256. Oshima M, Endoh M, Endo TA, et al. Genome-wide analysis of target genes regulated by HoxB4 in hematopoietic stem and progenitor cells developing from embryonic stem cells. Blood. 2011;117(15):e142-150.

257. Fan R, Bonde S, Gao P, et al. Dynamic HoxB4-regulatory network during embryonic stem cell differentiation to hematopoietic cells. Blood.

2012;119(19):e139-147.

258. Oguro H, Ding L, Morrison SJ. SLAM Family Markers Resolve Functionally Distinct Subpopulations of Hematopoietic Stem Cells and Multipotent Progenitors. Cell Stem Cell. 2013;13(1):102-116.

259. Deluche L, Joha S, Corm S, et al. Cryptic and partial deletions of PRDM16 and RUNX1 without $\mathrm{t}(1 ; 21)(\mathrm{p} 36 ; \mathrm{q} 22)$ and/or RUNX1-PRDM16 fusion in a case of progressive chronic myeloid leukemia: a complex chromosomal rearrangement of underestimated frequency in disease progression? Genes Chromosomes Cancer. 2008;47(12):1110-1117.

260. Duhoux FP, Ameye G, Montano-Almendras CP, et al. PRDM16 (1p36) translocations define a distinct entity of myeloid malignancies with poor prognosis but may also occur in lymphoid malignancies. Br J Haematol. 2012;156(1):76-88.

261. Zhou S, Ma Z, Lu T, Janke L, Gray JT, Sorrentino BP. Mouse transplant models for evaluating the oncogenic risk of a self-inactivating XSCID lentiviral vector. PLoS One. 2013;8(4):e62333.

262. Bunting KD, Galipeau J, Topham D, Benaim E, Sorrentino BP. Effects of retroviral-mediated MDR1 expression on hematopoietic stem cell self-renewal and differentiation in culture. Ann N Y Acad Sci. 1999;872:125-140; discussion 140-121.

263. Zhang CC, Lodish HF. Murine hematopoietic stem cells change their surface phenotype during ex vivo expansion. Blood. 2005;105(11):4314-4320.

264. Takiguchi M, Dow LE, Prier JE, et al. Variability of inducible expression across the hematopoietic system of tetracycline transactivator transgenic mice. PLoS One. 2013;8(1):e54009.

265. Groschel S, Lugthart S, Schlenk RF, et al. High EVI1 expression predicts outcome in younger adult patients with acute myeloid leukemia and is associated with distinct cytogenetic abnormalities. J Clin Oncol. 2010;28(12):2101-2107.

266. Buonamici S, Li D, Chi Y, et al. EVI1 induces myelodysplastic syndrome in mice. J Clin Invest. 2004;114(5):713-719.

267. Eguchi-Ishimae M, Eguchi M, Ohyashiki K, Yamagata T, Mitani K. Enhanced expression of the EVI1 gene in NUP98/HOXA-expressing leukemia cells. Int J Hematol. 2009;89(2):253-256.

268. Kurokawa M, Mitani K, Yamagata T, et al. The evi-1 oncoprotein inhibits c-Jun $\mathrm{N}$-terminal kinase and prevents stress-induced cell death. EMBO J. 2000;19(12):2958-2968.

269. Buonamici S, Li D, Mikhail FM, et al. EVI1 abrogates interferon-alpha response by selectively blocking PML induction. J Biol Chem. 2005;280(1):428-436.

270. Yoshimi A, Goyama S, Watanabe-Okochi N, et al. Evil represses PTEN expression and activates $\mathrm{PI} 3 \mathrm{~K} / \mathrm{AKT} / \mathrm{mTOR}$ via interactions with polycomb proteins. Blood. 2011;117(13):3617-3628. 
271. Yoshimi A, Kurokawa M. Evil forms a bridge between the epigenetic machinery and signaling pathways. Oncotarget. 2011;2(7):575-586.

272. Falini B, Mecucci C, Tiacci E, et al. Cytoplasmic nucleophosmin in acute myelogenous leukemia with a normal karyotype. N Engl J Med. 2005;352(3):254266.

273. Delhommeau F, Dupont S, Della Valle V, et al. Mutation in TET2 in myeloid cancers. N Engl J Med. 2009;360(22):2289-2301.

274. Kottaridis PD, Gale RE, Frew ME, et al. The presence of a FLT3 internal tandem duplication in patients with acute myeloid leukemia (AML) adds important prognostic information to cytogenetic risk group and response to the first cycle of chemotherapy: analysis of 854 patients from the United Kingdom Medical Research Council AML 10 and 12 trials. Blood. 2001;98(6):1752-1759.

275. Hemmat M, Chen W, Anguiano A, et al. Submicroscopic deletion of 5q involving tumor suppressor genes (CTNNA1, HSPA9) and copy neutral loss of heterozygosity associated with TET2 and EZH2 mutations in a case of MDS with normal chromosome and FISH results. Mol Cytogenet. 2014;7:35.

276. Jankowska AM, Szpurka H, Tiu RV, et al. Loss of heterozygosity 4q24 and TET2 mutations associated with myelodysplastic/myeloproliferative neoplasms. Blood. 2009;113(25):6403-6410.

277. Schnittger S, Dicker F, Kern W, et al. RUNX1 mutations are frequent in de novo AML with noncomplex karyotype and confer an unfavorable prognosis. Blood. 2011;117(8):2348-2357.

278. Holme H, Hossain U, Kirwan M, Walne A, Vulliamy T, Dokal I. Marked genetic heterogeneity in familial myelodysplasia/acute myeloid leukaemia. $\mathrm{Br} J$

Haematol. 2012;158(2):242-248. 


\section{VITA}

Hui Yu was born in Nantong, Jiangsu Province in P.R.China in 1981. In 2000 she was enrolled in China Medical University. After receiving her Bachelor's Degree of Medicine in Nursing in 2000, she joined Master's Program in Otolaryngology in Fudan University in China and finished her study in 2008. In the same year she was admitted to Integrated Program in Biomedical Sciences at the University of Tennessee Health Science Center to pursue her PhD degree. In 2009 she joined Dr. Brian P. Sorrentino's lab at St. Jude Children's Research Hospital and conducted research of hematopoietic stem cell self-renewal and differentiation. Her study aimed to uncover the mechanism of HOXB4-induced hematopoietic stem cell expansion and Prdm16's role in HSC selfrenewal and leukemogenesis under the HOXB4 overexpression context in vivo. She is expected to finish her work and graduate in December 2014. 